\title{
Measuring vortical flows in the solar interior
}

\author{
Dissertation \\ zur Erlangung des mathematisch-naturwissenschaftlichen Doktorgrades \\ "Doctor rerum naturalium" \\ der Georg-August-Universität Göttingen \\ im Promotionsprogramm ProPhys \\ der Georg-August University School of Science (GAUSS)
}

\author{
vorgelegt von \\ Jan Langfellner \\ aus Hann. Münden
}

Göttingen, 2015 
Betreuungsausschuss

Prof. Dr. Laurent Gizon

Max-Planck-Institut für Sonnensystemforschung und

Institut für Astrophysik, Georg-August-Universität, Göttingen, Deutschland

Prof. Dr. Stefan Dreizler

Institut für Astrophysik, Georg-August-Universität, Göttingen, Deutschland

Dr. Robert Cameron

Max-Planck-Institut für Sonnensystemforschung, Göttingen, Deutschland

Mitglieder der Prüfungskommission

Referent: Prof. Dr. Laurent Gizon

Max-Planck-Institut für Sonnensystemforschung und

Institut für Astrophysik, Georg-August-Universität, Göttingen, Deutschland

Korreferent: Prof. Dr. Stefan Dreizler

Institut für Astrophysik, Georg-August-Universität, Göttingen, Deutschland

2. Korreferent: Prof. Dr. Dean-Yi Chou

Astrophysics Laboratory, National Tsing Hua University, Taiwan

Weitere Mitglieder der Prüfungskommission:

Prof. Dr. Ulrich Christensen

Max-Planck-Institut für Sonnensystemforschung, Göttingen, Deutschland

Prof. Dr. Thorsten Hohage

Institut für Numerische und Angewandte Mathematik, Georg-August-Universität, Göttingen, Deutschland

Prof. Dr. Manfred Schüssler

Max-Planck-Institut für Sonnensystemforschung, Göttingen, Deutschland

PD Dr. Olga Shishkina

Max-Planck-Institut für Dynamik und Selbstorganisation, Göttingen, Deutschland

Tag der mündlichen Prüfung: 27. Juli 2015 


\section{Contents}

$\begin{array}{ll}\text { Summary } & 7\end{array}$

\begin{tabular}{ll}
\hline Zusammenfassung & 9
\end{tabular}

\begin{tabular}{ll}
\hline Preamble & 11
\end{tabular}

\begin{tabular}{lll}
\hline & Introduction & 13
\end{tabular}

1.1 The $\operatorname{Sun} \ldots \ldots \ldots \ldots \ldots \ldots \ldots$

1.1 .1 Solar structure $\ldots \ldots \ldots \ldots$. . . . . . . . . . . . . . . 13

1.1 .2 The solar cycle . . . . . . . . . . . . . . . . . . 14

1.1 .3 Convection . . . . . . . . . . . . . . . . . . . . . . . . . . . . . .

1.1 .4 Oscillations . . . . . . . . . . . . . . . . . . 17

1.1 .5 Influence of solar rotation on convection . . . . . . . . . . . 17

1.2 The supergranulation mystery $\ldots \ldots \ldots \ldots$. . . . . . . . . . 18

1.3 Scope of the thesis $\ldots \ldots \ldots \ldots \ldots$

1.4 Helioseismology . . . . . . . . . . . . . . . . . . . . 20

1.4.1 Global helioseismology . . . . . . . . . . . . . . . 20

1.4.2 Local helioseismology . . . . . . . . . . . . . . . 21

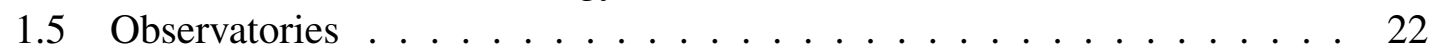

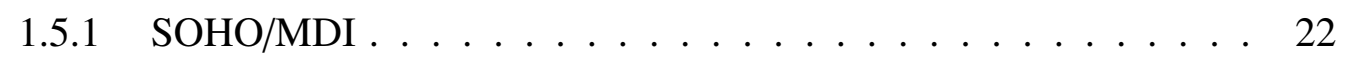

1.5 .2 SDO/HMI $\ldots \ldots \ldots \ldots \ldots \ldots \ldots$

1.5 .3 Other observatories . . . . . . . . . . . . . . . . 24

2 Paper I: Time-distance helioseismology: A new averaging scheme for measuring flow vorticity 25

2.1 Introduction . . . . . . . . . . . . . . . . . 25

2.2 Measuring vortical flows along a closed contour . . . . . . . . . . . . 27

2.2 .1 Geometry for anti-clockwise travel times . . . . . . . . . . 27

2.2 .2 Reducing the noise level . . . . . . . . . . . . . . . . . 28

2.2 .3 Decoupling from isotropic wave-speed perturbations . . . . . . . 30

2.3 Proof of concept using SDO/HMI and SOHO/MDI observations . . . . 30

2.3 .1 Observations . . . . . . . . . . . . . 30

2.3 .2 Travel-time maps . . . . . . . . . . . . . . . 31

2.3.3 Test 1: Evidence of a vorticity signal in $\tau^{\text {ac }}$ as a function of

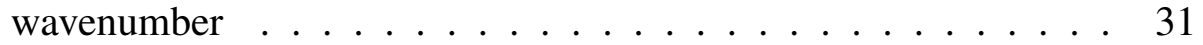

2.3 .4 Test 2: Effect of rotation on vorticity in supergranules . . . . . . . 33 
2.4 Conclusion $\ldots \ldots \ldots \ldots \ldots \ldots$

3 Paper II: Spatially resolved vertical vorticity in solar supergranulation using helioseismology and local correlation tracking 39

3.1 Introduction . . . . . . . . . . . . . . . . . . . 40

3.1.1 Time-distance helioseismology . . . . . . . . . . . . . . . 40

3.1 .2 Local correlation tracking . . . . . . . . . . . . . . . . 41

3.2 Observations and data processing . . . . . . . . . . . . . . 41

$3.2 .1 \quad$ Flow velocity maps from local correlation tracking . . . . . . . . 43

3.2.2 Travel-time maps for horizontal divergence and vertical vorticity . 44

3.3 Comparison of horizontal divergence and vertical vorticity from TD and

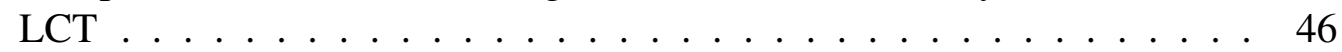

3.3.1 Spatial power spectra of horizontal divergence and vertical vorticity 46

3.3.2 Maps of horizontal divergence and vertical vorticity. . . . . . . . 47

3.4 Net vortical flows in the average supergranule . . . . . . . . . . . . 51

3.4.1 Obtaining maps of the average supergranule . . . . . . . . . . 51

\begin{tabular}{|lll}
\hline 3.4 .2 & Latitudinal dependence of the vertical vorticity in outflow regions 53
\end{tabular}

3.4 .3 Inflow regions $\ldots \ldots \ldots \ldots 6$

3.4 .4 Dependence of the vertical vorticity on horizontal divergence. . . 56

3.4 .5 Comparison of SDO/HMI and SOHO/MDI . . . . . . . . . . 60

3.5 Differences between outflow and inflow regions . . . . . . . . . . . . 61

$3.6 \quad$ Radial and tangential velocities versus radial distance . . . . . . . . . . . . 63

3.7 Summary . . . . . . . . . . . . . . . . . . . 64

3.7 .1 Validation . . . . . . . . . . . . . . . . 64

3.7 .2 Scientific results: spatial maps of vertical vorticity . . . . . . . 66

3.8 Appendix: Ridge filters . . . . . . . . . . . . . . . . . . . . 67

$3.9 \quad$ Appendix: Conversion of travel times into flow velocities . . . . . . . . 68

3.10 Appendix: Systematic errors . . . . . . . . . . . . . . . . . 69

3.10 .1 Center-to-limb systematics . . . . . . . . . . . . . . . . . . 69

3.10 .2 MDI instrumental systematics . . . . . . . . . . . . . . . . . 72

3.10 .3 Selection of filter and $\tau^{\mathrm{ac}}$ geometry parameters . . . . . . . 72

4 Paper III: Anisotropy of the solar network magnetic field around the average $\begin{array}{lr}\text { supergranule } & \mathbf{7 5}\end{array}$

4.1 Introduction . . . . . . . . . . . . . . . . . . . . 75

4.2 Observations and data processing . . . . . . . . . . . . . . . . . . 76

4.3 Results . . . . . . . . . . . . . . . . 76

4.3.1 Magnetic field of the average supergranule near disk center . . . . 76

4.3.2 Measuring the anisotropy of the network field . . . . . . . . . . 80

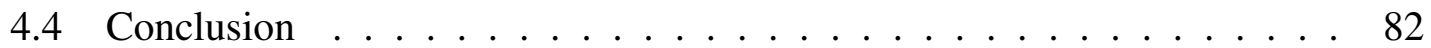

\begin{tabular}{lll}
\hline & Discussion & $\mathbf{8 3}$
\end{tabular}

5.1 Toy models for vortical flows $\ldots \ldots \ldots$. . . . . . . . . 83

5.2 Outlook . . . . . . . . . . . . . . . . . . 87

\begin{tabular}{lr}
\hline Bibliography & 89
\end{tabular} 
\begin{tabular}{ll}
\hline Appendices & 99
\end{tabular}

\begin{tabular}{|lr}
\hline A Time-distance measurements & 101
\end{tabular}

A.1 Ridge filter construction . . . . . . . . . . . . . . . . . . . 101

A.2 Correlation of rotated $\tau^{\mathrm{ac}}$ measurements . . . . . . . . . . . . . 103

\begin{tabular}{llr}
\hline B Velocity and vorticity measurements & 109
\end{tabular}

B.1 Center-to-limb systematics . . . . . . . . . . . . . . . . . . . . . . 109

B.2 $\quad$ Average supergranular outflow and inflow profiles . . . . . . . . . . 115

\begin{tabular}{ll}
\hline Magnetic field measurements & 117
\end{tabular}

C.1 Location of the network magnetic field . . . . . . . . . . . . . . . . . . 117

C.2 Relation between magnetic field and mean travel times . . . . . . . . . . 118

C.3 Center-to-limb systematics . . . . . . . . . . . . . . . . . . 118

C.4 Magnetic field at the average supergranular inflow . . . . . . . . . . . . . 123

\begin{tabular}{lr}
\hline Publications & 125
\end{tabular}

\begin{tabular}{|l|l}
\hline Acknowledgements & 127
\end{tabular}

\begin{tabular}{ll}
\hline Curriculum vitae & 129
\end{tabular} 



\section{Summary}

This thesis focuses on observations of the effects of rotation on solar convection at the length scales of supergranulation and larger $(>30 \mathrm{Mm})$. Rotation drives vortical flows through the Coriolis force and causes anisotropic velocity correlations that are believed to influence the large-scale solar dynamics.

We obtain horizontal flows using photospheric Doppler velocity and continuum intensity images from the Helioseismic and Magnetic Imager (HMI) onboard the Solar Dynamics Observatory (SDO) spacecraft via the techniques of time-distance helioseismology (TD) and local correlation tracking (LCT) of granules. In time-distance helioseismology, the local vertical vorticity can be measured by taking the difference between wave travel times measured in the anti-clockwise and clockwise directions along a closed contour. The agreement between the TD and LCT methods is excellent up to $\pm 60^{\circ}$ latitude, provided that a center-to-limb correction is applied.

Averaging over longitude, one finds that there is a small but significant correlation between the horizontal divergence and the vertical vorticity component of supergranular flows away from the solar equator. By comparison to a noise model, we find that the TD technique can be used to probe the vertical vorticity of flows on spatial scales larger than about $15 \mathrm{Mm}$, thus including supergranules and also giant cells. We also find that the vertical vorticity signal is much easier to measure using SDO/HMI observations than previous observations.

The impact of the Sun's rotation on supergranulation is studied in detail by making spatial maps of the vertical vorticity of the flows associated with the average supergranule. The average supergranule is constructed by co-aligning thousands of individual supergranules in a given latitude band. For the first time, we are able to spatially resolve vorticity associated with inflows and outflow regions. In the northern hemisphere, outflows are on average associated with a clockwise circulation. The signal vanishes at the equator and has opposite sign in the southern hemisphere. Inflow and outflow regions have vertical vorticity of opposite sign, as expected from predictions based on the effects of the Coriolis force. The peak of the vertical vorticity in the average supergranular outflow region is rather broad and weak (full width at half maximum, FWHM, of $13 \mathrm{Mm}$ and peak value of $4 \times 10^{-6} \mathrm{~s}^{-1}$ clockwise at $40^{\circ}$ latitude) compared to the average inflow region ( $8 \mathrm{Mm}$ FWHM and peak value of $8 \times 10^{-6} \mathrm{~s}^{-1}$ anti-clockwise).

Furthermore, we study the magnetic field around the average supergranule (in the inflow regions) at the equator using SDO/HMI observations. We discover an anisotropy in the average magnetic field strength, which is larger in the west (prograde) than in the east by about $10 \%$. This surprising result adds to the mystery of solar supergranulation. Whether it is connected to other supergranular properties, such as pattern superrotation or wavelike properties, is unclear. 



\section{Zusammenfassung}

Diese Dissertation befasst sich mit Beobachtungen von konvektiven Strömungen in der Sonne, und insbesondere mit den Auswirkungen der Rotation auf diese Strömungen auf der Längenskala von Supergranulation und größeren Skalen (>30 Mm). Die Rotation der Sonne verursacht durch die Corioliskraft Wirbelströmungen und bewirkt anisotrope Korrelationen der Geschwindigkeitskomponenten. Man nimmt an, dass diese Korrelationen die Dynamik der Sonne auf großen Längenskalen beeinflussen.

Um horizontale Strömungen zu messen, untersuchen wir photosphärische Aufnahmen der Doppler-Geschwindigkeit und der Kontinuumsintensität des "Helioseismic and Magnetic Imagers" (HMI) an Bord der Raumsonde "Solar Dynamics Observatory" (SDO) mit Hilfe der Methoden Time-Distance-Helioseismologie (TD) und Local Correlation Tracking (LCT) von Granulen. Im Rahmen der Time-Distance-Helioseismologie kann die lokale vertikale Vortizität gemessen werden, indem die Differenz von Wellenlaufzeiten entlang eines geschlossenen Weges ermittelt wird (Laufzeiten gegen den Uhrzeigersinn minus Laufzeiten im Uhrzeigersinn). Die Ergebnisse von TD und LCT stimmen bis $\mathrm{zu}$ den höchsten studierten Breitengraden $\left( \pm 60^{\circ}\right)$ hervorragend überein, nachdem eine Korrektur für so genannte Center-to-Limb-Effekte angewandt wurde.

Nach dem Mitteln in Ost-West-Richtung messen wir abseits des Äquators eine schwache, aber signifikante Korrelation zwischen der horizontalen Komponente der Divergenz und der vertikalen Komponente der Vortizität von supergranularen Strömungen. Ein Vergleich der Messungen mit einem Modell für das Rauschen offenbart, dass die TD-Methode verwendet werden kann, um die vertikale Vortizität von Strömungen auf Längenskalen größer als $15 \mathrm{Mm}$ zu messen. Damit können mit dieser Methode nicht nur Strömungen in Supergranulen, sondern auch in Riesenzellen gemessen werden. Wir stellen außerdem fest, dass das Signal in Messungen der vertikalen Vortizität mit Hilfe von Aufnahmen von SDO/HMI sehr viel leichter detektiert werden kann als mit Hilfe von früheren Aufnahmen.

Um den Einfluss der Sonnenrotation auf die Supergranulation im Detail zu studieren, kartieren wir die vertikale Vortizität der Strömungen in der durchschnittlichen Supergranule. Die durchschnittliche Supergranule wird konstruiert, indem Tausende von einzelnen Supergranulen in einem bestimmten Breitengradbereich durch räumliche Verschiebungen zur Deckung gebracht werden. Damit lösen wir zum ersten Mal die vertikale Vortizität in Aus- und Einströmungen räumlich auf. In nördlichen Breiten sind Ausströmungen im Mittel mit einer Zirkulation im Uhrzeigersinn verbunden. Das Signal verschwindet am Äquator und hat in südlichen Breiten das umgekehrte Vorzeichen. Aus- und Einströmungen besitzen eine vertikale Vortizität mit entgegengesetzten Vorzeichen, wie es von Vorhersagen erwartet wird, die sich auf die Corioliskraft stützen. Es wird offenbar, dass der Vortizitätspeak in der durchschnittlichen supergranularen Ausströmung vergleichs- 
weise ausgedehnt und schwach ist (Halbwertsbreite von $13 \mathrm{Mm}$ und Spitzenwert von $4 \times 10^{-6} \mathrm{~s}^{-1}$ im Uhrzeigersinn bei $40^{\circ}$ nördlicher Breite), verglichen mit der durchschnittlichen Einströmung (Halbwertsbreite von $8 \mathrm{Mm}$ und Spitzenwert von $8 \times 10^{-6} \mathrm{~s}^{-1}$ gegen den Uhrzeigersinn).

Darüberhinaus untersuchen wir mit SDO/HMI-Daten das Magnetfeld in den Einströmungen um die durchschnittliche Supergranule am Äquator herum. Die mittlere Stärke des Magnetfelds stellt sich als richtungsabhängig heraus: In westlicher Richtung (prograd) ist das Netzwerkfeld ungefähr 10\% stärker als in östlicher Richtung. Dieses überraschende Ergebnis fügt dem Rätsel um die Supergranulation einen weiteren Aspekt hinzu. Ob ein Zusammenhang mit anderen bekannten Eigenschaften der Supergranulation besteht (beispielsweise zur Superrotation des supergranularen Strömungsmusters oder zu wellenartigen Eigenschaften), ist nicht geklärt. 


\section{Preamble}

This thesis is presented in the cumulative format ("kumulative Dissertation"), as specified in the rules of the Georg-August University School of Science (GAUSS), §10(5). The main chapters of the thesis (except "Introduction" and "Discussion") reproduce the following scientific journal articles:

- Paper I:

J. Langfellner, L. Gizon, A. C. Birch: Time-distance helioseismology: A new averaging scheme for measuring flow vorticity, Astronomy \& Astrophysics 570, A90, 2014

- Paper II:

J. Langfellner, L. Gizon, A. C. Birch: Spatially resolved vertical vorticity in solar supergranulation using helioseismology and local correlation tracking, Astronomy \& Astrophysics 581, A67, 2015

- Paper III:

J. Langfellner, L. Gizon, A. C. Birch: Anisotropy of the solar network magnetic field around the average supergranule, Astronomy \& Astrophysics 579. L7, 2015. 



\section{Introduction}

With the development of powerful telescopes and observational techniques, a wealth of phenomena have been discovered in the Sun over the course of the past centuries - from granulation to the magnetic cycle, flares and the solar wind. This work focuses on the phenomenon of supergranulation - convection cells that have a diameter of about $30000 \mathrm{~km}$ each, live for one to two days, and cover the entire solar surface. Supergranulation is a rather mysterious phenomenon, whose nature has remained elusive. In this thesis, the influence of solar rotation on supergranulation is studied, from an observational point of view. We will show that rotation imposes a preferred sense of rotation on the horizontal outflows and inflows that can be spatially resolved.

In the following, we briefly introduce solar physics, with a focus on convection. We then describe methods of local helioseismology, which can be used for measuring supergranular flows in the solar interior. Furthermore, we introduce space-borne observatories that provide the necessary high-definition data for the work described in this thesis.

\subsection{The Sun}

Our central star is a hydrogen-burning main-sequence star in the middle of its life cycle, with an age of about 4.6 billion years (e.g., Houdek \& Gough 2011). It has a radius, in the following denoted by the symbol $R_{\odot}$, of about $696 \mathrm{Mm}$ (or $696000 \mathrm{~km}$ ), which is more than 100 times the Earth's radius. At the same time, the solar mean density is $1.4 \mathrm{~g} \mathrm{~cm}^{-3}$, only a quarter of the Earth's value. The Sun's effective temperature is about 5778 kelvins (e.g., Stix 2002).

\subsubsection{Solar structure}

The Sun can be divided in several regions that surround its center like the shells of an onion. The solar interior (the part of the Sun that is not directly visible) consists of the core, the radiative zone, and the convection zone. The core is the innermost part of the Sun, with temperatures of about 15 million kelvins and densities up to $100 \mathrm{~g} \mathrm{~cm}^{-3}$. The physical conditions in the core are extreme enough to allow for nuclear fusion processes to take place. Hydrogen is converted into helium via different nuclear reactions, predominantly the proton-proton chain. The excess energy that is released by the nuclear fusion is transported outward via different mechanisms. Some energy is carried away by neutrinos, which only marginally interact with the solar matter. Apart from this, the energy transport in the core and the subsequent radiative zone is dominated by photons. Energy-rich $\gamma$ rays are scattered numerous times and are gradually converted into photons with less 
energy. Due to a mean free path of only a few millimeters, the energy transport is highly diffusive. At about $0.7 R_{\odot}$, energy transport by bulk fluid motions (convection) becomes more effective and constitutes the dominant energy transport mechanism up to the solar surface (e.g., Christensen-Dalsgaard et al. 1991).

The transition between the radiative and the convective zones coincides with a sudden change in solar rotation behavior. Whereas the Sun rotates like a solid body in the radiative zone, the solar rotation rate depends on latitude and depth in the convection zone - a behavior named differential rotation. The shear layer that separates the two regimes of rotation is called tachocline (e.g., Spiegel \& Zahn 1992). Another radial shear zone is present just below the solar surface, down to a depth of about $30 \mathrm{Mm}$. In this near-surface shear layer, the rotation rate increases with depth (e.g., Schou et al. 1998). At the surface, the solar sidereal (i.e., with respect to the sky background) rotation period varies between about 25 days at the equator and roughly 35 days near the poles (for a review on solar rotation, see Howe 2009).

At the top end of the solar convection zone, the density decreases quickly (to about $10^{-7} \mathrm{~g} \mathrm{~cm}^{-3}$ ) and light can escape into space. Thus this layer, the so-called photosphere, is directly accessible to observations. The photosphere measures only a few hundred kilometers in radial direction, but most of the light that reaches us from the Sun is emitted in this region. At the top of the photosphere, the density keeps decreasing, whereas the temperature reaches a minimum of about $4000 \mathrm{~K}$ and starts to increase again outward. In the subsequent two megameters, the so-called chromosphere, the temperature increases by about an order of magnitude. Further outward, the temperature keeps increasing to more than one million kelvins in the corona. How these temperatures are reached, is currently not understood (see, e.g., Parnell \& De Moortel 2012, for a review). Between the chromosphere and the corona, the transition region is located, which is heated by the hot corona due to thermal conduction.

Beyond the corona, the solar atmosphere continues in the form of highly energetic and magnetized particles that leave the Sun at a speed of several hundreds kilometers per second. This solar wind reaches beyond all known planets of the solar system, until it hits the interstellar medium. The region that is dominated by the solar wind is called heliosphere.

For a more detailed introduction to the Sun, see, for example, Kippenhahn \& Weigert (1990) or Stix (2002).

\subsubsection{The solar cycle}

The solar structure is not static. Plasma flows and magnetic fields in the solar atmosphere are highly dynamic with energy being released in violent eruptions like coronal mass ejections, which can affect the Earth, for example in the form of induced currents in power grids, which can cause damage. Long-time observations of the Sun reveal an (approximately) 11-year activity cycle, during which the number of sunspots changes drastically. At the beginning of each cycle, sunspots usually start to appear in both hemispheres at a latitude of about $30^{\circ}$. The number of sunspots keeps increasing and the points where they emerge slowly move toward the equator from both north and south. When the two branches of sunspots finally meet, the emergence of new sunspots stops and a new cycle begins. With each activity cycle, the global (poloidal) magnetic field reverses its poles, so 
a full magnetic cycle takes 22 years.

How exactly the solar cycle is maintained is unclear. There exist many different models that attempt to explain the solar cycle as a hydromagnetic dynamo process. One classical model is the Babcock-Leighton dynamo (Babcock 1961, Leighton 1964, 1969). The differential rotation in the solar convection zone can convert poloidal (north-south) magnetic field into toroidal (east-west) field. The magnetic field might be condensed at the tachocline and become buoyantly unstable, thus rising in the form of flux tubes to the solar surface. There, the flux tubes form bipolar active regions and become visible as sunspots. Most bipolar regions in a hemisphere have the same leading (west) and trailing (east) polarities (Hale's law). On average, bipolar regions are tilted toward the equator (Joy's law). Over time, most bipolar regions are dissociated by turbulent convection and the opposite magnetic field polarities cancel. However, some bipolar regions might halfway cross the equator, where the leading and trailing polarities are separated due to the meridional circulation, which transports material (and magnetic flux) poleward in both hemispheres in opposite directions like conveyor belts. Because the leading polarity has the opposite sign of the polar field, this process can gradually revert the polar field, especially when strong active regions are involved. Recent support for the Babcock-Leighton dynamo has been provided by, e.g., Cameron \& Schüssler (2015).

Another class of dynamo models, the mean-field models (see, e.g., Krause \& Rädler 1980), follow the idea that small-scale fast evolving processes (turbulent convection) can be separated from large-scale, slowly changing processes (rotation, meridional circulation). In this picture, the small-scale processes only contribute to the dynamo action through net effects (non-vanishing mean). This usually boils down to so-called $\alpha-\Omega$ dynamos: Poloidal magnetic field is converted into toroidal field by the differential rotation $(\Omega$ effect) and toroidal field is converted into poloidal field with the opposite orientation through turbulent convection that is affected by solar rotation ( $\alpha$ effect).

However, all dynamo models have their specific flaws and oversimplify the dynamics. For an extensive review, see, e.g., Charbonneau (2010).

In the following, some aspects of solar physics are highlighted that are particularly relevant for this thesis: convection (and especially supergranulation), solar oscillations, and the interaction between convection and rotation.

\subsubsection{Convection}

In the outer $30 \%$ of the solar radius, the energy transport is dominated by convection. In a simplified picture, a fluid parcel that is (by whatever chance) slightly pushed upward and expands adiabatically (due to the temperature gradient) becomes buoyant, which increases the upward acceleration. For this to happen, the density of the expanding fluid parcel has to decrease stronger with height than the density in the surroundings. This condition is called Schwarzschild criterion.

However, the fluid parcel cannot expand and accelerate all the way up to the surface but rather starts to mix with its surroundings. Mixing-length theory (Böhm-Vitense 1958) is based on the assumption that the material of the fluid parcel completely mixes with the surroundings after a certain vertical distance - the mixing length. Typically, the mixing length is given as a fraction $\alpha$ of the pressure scale height, with $\alpha \sim 1$.

Because of mass conservation, such convective upflows have to be balanced by down- 
flows. This leads to the formation of convection cells, in which hot material is brought upward, mixes with its cooler surroundings (and thus deposits heat), and sinks as cooler, denser material to complete the cycle. As the net outcome, energy is transported upward, whereas there is no net mass transport.

In the photosphere, in sequences of continuum images in the visible part of the electromagnetic spectrum, convective structures of size 1 to $2 \mathrm{Mm}$ stand out, the so-called granules (for a detailed review on granulation and solar near-surface convection in general, see Nordlund et al.|2009). They are characterized by bright centers and darker edges, the intergranular lanes, and are highly dynamic, with a lifetime of about 5 to $10 \mathrm{~min}$. In line with the theory, hot material is brought up from deeper down in the bright granule centers. Due to mass conservation and the rapidly decreasing density with increasing height, the upflow is turned into a horizontal outflow at the top of the granule. The opacity in the photosphere decreases to a point that allows effective energy transport through radiation, in the form of light escaping to space. The bright, hot plasma in the granule centers thus cools down and gathers as darker, denser material in the intergranular lanes, where it flows downward due to negative buoyancy. It is rather this cooling process that is thought to predominantly drive the convection than the heating from the bottom (e.g., Stein \& Nordlund 1989, Spruit|1997, Stein et al.|2009). The horizontal flow velocities associated with granulation reach several kilometers per second, sometimes exceeding the sound speed (about $7 \mathrm{~km} \mathrm{~s}^{-1}$, e.g., Nordlund et al.2009) and forming shock waves (Rybák et al. 2004). In regions where the magnetic field is comparably weak, the so-called quiet Sun, the magnetic field is tied to these vigorous flows and usually follows the granular motions. Thus magnetic field lines tend to concentrate in the intergranular lanes, especially at the junctions (for a review that focuses on the role of the magnetic field in quiet-Sun convection, see Stein 2012). In active regions, where the magnetic fields reach strengths of a few thousand Gauss in extended areas, on the other hand, convection can be severely impeded, leading to darker features like sunspots (see, e.g., Rempel \& Schlichenmaier 2011, for a review).

The strong stratification of the convection zone, spanning many density scale heights, has (together with mixing-length theory) led to the picture of a cascade of convection cells rather than a single layer of cells reaching from the tachocline to the photosphere. In this view, the granules merely form the top row of the cascade. Because the density scale height increases quickly with depth, the deeper cells are thought to be much larger than the granules, while the flow speed is reduced. This picture is supported by numerical simulations of convection (e.g., Stein \& Nordlund 1989).

Observations, on the other hand, show a somewhat more complex picture that escapes any easy interpretation. Measurements of the convective velocity power spectrum in the photosphere (Hathaway et al. 2000) indeed show a broad distribution of power at larger spatial scales than granulation. A prominent peak in velocity power occurs at a scale of about $30 \mathrm{Mm}$ with flow speeds of about $300 \mathrm{~m} \mathrm{~s}^{-1}$, associated with a phenomenon named supergranulation. In spite of the suggestive name, however, the origin of supergranulation is still unclear. Furthermore, convection simulations so far have failed to reproduce the observed power peak that marks supergranulation as a special spatial flow scale. A more detailed discussion of supergranulation is given in Sect.1.2. The largest convection cells, which have been detected only recently (Hathaway et al. 2013), are dubbed giant cells. They reach a diameter of about $200 \mathrm{Mm}$ and flow speeds of roughly $10 \mathrm{~m} \mathrm{~s}^{-1}$. 
Our current understanding of solar convection is not only incomplete near the surface, but especially limited in the deeper parts of the convection zone. Hanasoge et al. (2012), making use of time-distance helioseismology, obtained an upper limit of the convective flow speed $\left(\sim 1 \mathrm{~m} \mathrm{~s}^{-1}\right)$ at depths of $30 \mathrm{Mm}$ that is two orders of magnitude below values from simulations. If true, the convective flows would be too weak to explain the observed differential rotation with angular momentum transport due to Reynolds stress (Miesch et al. 2012). A model of convective flow structure that might be consistent with the measurements by Hanasoge et al. (2012) suggests collimated downflows that are below the resolution limit of the helioseismology measurements (Spruit 1997). However, other authors report stronger flows that are compatible with the simulation results (Greer et al. 2015). This disagreement has not been resolved yet.

\subsubsection{Oscillations}

The vigorous near-surface convection continuously and stochastically excites waves (e.g., Goldreich \& Keeley 1977, Stein \& Nordlund 2001) that can be detected at the surface as, for example, brightness variations or fluctuations of the line-of-sight velocity of the plasma (Leighton et al. 1962). The typical period of these oscillations is about five minutes. Some waves are enhanced and form standing waves, as the Sun constitutes a spherical resonance body, where most waves are trapped between the surface (the upper turning point) and some depth that depends on the wavelength (the lower turning point). The different standing waves are called normal modes and can be described by a set of three quantum numbers, $n, \ell$, and $m$. The quantum number $n$ describes how many nodes there are in radial direction (radial order), whereas $\ell$ gives the number of node lines in horizontal direction (degree of the mode) and $m$ determines how many of the horizontal node lines are in longitudinal direction. The oscillation pattern of the modes at the surface can be described by spherical harmonics.

In general, there are three different types of modes: The pressure modes ("p modes") are formed by acoustic waves. The gravity modes ("g modes") are supported in areas where fluid parcels are buoyantly stable, i.e., there is no convection but the fluid parcels oscillate around their equilibrium positions. Such conditions are met in the radiative zone. The g modes are evanescent in the convection zone and thus have only small amplitudes at the surface. Therefore, it is difficult to observe g modes in the Sun, and claims of detection have been controversial (see, e.g., Appourchaux et al. 2010, for a discussion). The fundamental modes ("f modes") are formed by surface gravity waves that arise due to a sharp density gradient (possibly at the transition region, e.g., Rosenthal \& Gough 1994, Rosenthal \& Christensen-Dalsgaard 1995) and can be compared to deep water waves on Earth. The $\mathrm{f}$ modes have no nodes in radial direction $(n=0)$. For a review on solar oscillations, see, e.g, Christensen-Dalsgaard (2002).

\subsubsection{Influence of solar rotation on convection}

The solar rotation influences convection (see, e.g., Gizon et al. 2010). For instance, horizontal outflows of convective cells are deflected to the right in the northern hemisphere by the Coriolis acceleration,

$$
\mathbf{a}_{C}=-2 \boldsymbol{\Omega} \times \mathbf{v},
$$


where $\boldsymbol{\Omega}$ is the solar rotation and $\mathbf{v}$ the plasma flow velocity. This is similar to anticyclonic high-pressure regions in the Earth's weather system. However, this effect is concealed by the strong turbulence of the Sun's plasma. A measure of the relative strength of Coriolis force and inertial forces is the dimensionless Coriolis number, Co, given by

$$
\operatorname{Co}=2 \tau \Omega(\lambda) \sin (\lambda),
$$

with the lifetime $\tau$ of the convective feature and the solar rotation rate $\Omega(\lambda)$ dependent on the solar latitude $\lambda$. The latitudes and longitudes are given in the Carrington coordinate system, with the poles lying on the rotation axis. Note that the Coriolis number is the inverse of the widely used Rossby number. A large Coriolis number (Co $\gg 1)$ indicates that the Coriolis force dominates (as in hurricanes on Earth), whereas for Co $\ll 1$ turbulence effects prevail.

The latter is the case for granules with their lifetime of five minutes, whereas for giant cells, the Coriolis number is larger than one, as they live for months (Hathaway et al. 2013). Supergranules have a lifetime of about one or two days (e.g., Hirzberger et al. 2008, Roudier et al. 2014). As a consequence, the Coriolis number is of the order Co $\sim \sin (\lambda)$. This means that single supergranules at a given latitude are expected to rotate either way, but on average there should be a small net velocity in the preferred sense of rotation (clockwise for outflows and anti-clockwise for inflows in the northern hemisphere). This corresponds to a negative (positive) vertical component of flow vorticity, $\omega_{z}$, in outflows (inflows) in the northern hemisphere. As outflows correspond to a positive horizontal component of flow divergence, $\operatorname{div}_{h}$, and inflows to a negative $\operatorname{div}_{h}$, the average $\left\langle\operatorname{div}_{h} \omega_{z}\right\rangle$ over longitude at a given latitude strip should be negative in the northern hemisphere. Indeed, this has been measured for supergranules (Gizon et al. 2003) and giant cells (Hathaway et al. 2013).

The quantity $\left\langle\operatorname{div}_{h} \omega_{z}\right\rangle$ is a proxy for the vertical component $\left\langle v_{z} \omega_{z}\right\rangle$ of the net kinetic helicity, $\langle\mathbf{v} \cdot \boldsymbol{\omega}\rangle$ (Zeldovich et al. 1990, Rüdiger et al. 1999), with the vertical component of the flow velocity, $v_{z}$, and the vorticity, $\omega$. Helical flows can twist the magnetic field and might thus contribute to the $\alpha$ effect.

\subsection{The supergranulation mystery}

Solar supergranules were first detected by Hart (1954, 1956) in Doppler velocity images and measured to have a typical diameter of roughly $30 \mathrm{Mm}$ (Leighton et al. 1962). Simon \& Leighton (1964) found a strong correlation with both the chromospheric activity in the $\mathrm{Ca}$ II $\mathrm{K}$ line and the photospheric magnetic field. The Ca II $\mathrm{K}$ intensity and the magnetic field outline the supergranular cells as a network. The accumulation of magnetic field in the network can be explained by the advection of magnetic field due to the supergranular flows (e.g., Krijger \& Roudier 2003, Orozco Suárez et al.|2012), similar as for granules (Galloway et al. 1977). An extensive review of supergranulation is provided by Rieutord \& Rincon (2010).

Supergranular velocities are predominantly horizontal, with a magnitude of about $300 \mathrm{~m} \mathrm{~s}^{-1}$ (e.g., Simon \& Leighton 1964. Hathaway et al. 2002). The vertical velocity component is only of the order $\sim 10 \mathrm{~m} \mathrm{~s}^{-1}$ at the surface (e.g., Hathaway et al. 2002, Duvall \& Birch 2010). Typical lifetime estimates for supergranules are 1-2 days (e.g., 
Hirzberger et al. 2008, Roudier et al. 2014), but supergranules can also be tracked for longer times, up to one week (De Rosa \& Toomre 2004, Gizon 2006).

Why there is a peak in power spectra of horizontal velocities at a scale of $30 \mathrm{Mm}$ (see, e.g., Hathaway et al. 2000, Rieutord et al. 2010), is not clear. Among suggested explanations for a distinct peak at supergranulation scale are, for example, an enhanced opacity in certain depths due to helium or hydrogen ionization zones (e.g., Simon \& Leighton 1964, Schwarzschild 1975, Lord et al. 2014) or a non-linear interaction of convective flows and magnetic fields (Rieutord \& Rincon 2010). In local numerical simulations, no peak at supergranulation scale is found (e.g., Stein et al. 2006, Lord et al.2014), but this might be due to insufficient box sizes (horizontally or vertically) or missing physical ingredients. In

global simulations, supergranules have only recently become properly resolvable (Hotta et al.2014), but also without showing a peak.

Whereas the horizontal extension of supergranules has been successfully measured, the vertical extent is neither clearly predicted nor observationally determined. Different measurements reach from very shallow ( $\sim 1 \mathrm{Mm}$, e.g., Rieutord et al. 2010) to a significant fraction of the near-surface shear layer ( $\sim 15 \mathrm{Mm}$, Zhao \& Kosovichev 2003). Recently, Duvall \& Hanasoge (2013) and Duvall et al.(2014) found that the vertical velocity should increase with depth and peak at a depth of about $2 \mathrm{Mm}$, indicating a very shallow supergranulation structure.

As convective features, supergranules are associated with temperature perturbations that should be visible as an intensity contrast. In practice, such a temperature perturbation is, however, hard to detect in intensity images because it is necessary to disentangle enhanced intensity from temperature perturbations from enhanced intensity due to magnetic fields. Studies that included a careful treatment of magnetic fields (Rast 2003a, Meunier et al. 2007, Goldbaum et al.2009) found an intensity contrast corresponding to a temperature excess in supergranular outflows of $\sim 1 \mathrm{~K}$. This is about three orders of magnitude less than for granulation. However, the authors cannot exclude the possibility that unresolved, small-scale magnetic fields are responsible for the intensity excess on supergranulation scale.

There are also existing alternative models that challenge the convective origin of supergranulation. For instance, Rieutord et al. (2000) presented the idea that supergranulation is formed by the collective interaction of granules, in the form of non-linear coupled harmonic oscillators. Rast (2003b) suggested that granular downflows might merge to create downflows on supergranular scale. Crouch et al. (2007) developed a model, in which the random walk of magnetic elements plays the key role.

Various studies reported a higher rotation rate of the supergranulation pattern compared to granulation or magnetic features (e.g., Duvall 1980, Snodgrass \& Ulrich 1990, Meunier \& Roudier 2007). Gizon et al. (2003) and Schou(2003) found that supergranulation has wavelike properties and suggested that traveling-wave convection might explain the apparent superrotation.

\subsection{Scope of the thesis}

As discussed in the previous section, solar supergranulation is neither understood theoretically, nor has it been reproduced by numerical simulations, nor has it been studied 
comprehensively by observations. The interplay of solar rotation and supergranulation might have implications for the maintenance of differential rotation and meridional circulation, especially in the near-surface shear layer.

A major goal of this thesis is to spatially resolve the effect of the Coriolis force on supergranular outflows and inflows, in the form of a net vorticity (corresponding to a circular flow component) that remains after averaging over many supergranules, away from the solar equator. Furthermore, a profile of the average horizontal supergranular flows that includes rotational effects can serve as a template that will support future theoretical work, both modeling and simulations of supergranulation. The study of other observables like the magnetic field provides additional information on the relationship between supergranular flows and other physical quantities.

The task of mapping the vorticity in supergranular flows requires both appropriate data analysis methods and high-resolution observations. In terms of methods, the technique of time-distance helioseismology will be adapted to obtain maps of the vertical vorticity of supergranules and larger-scale flows in the solar interior, based on ideas used in ocean acoustic tomography on Earth. Establishing and testing this method in the solar context is the purpose of Paper I (Sect. 2). Paper II (Sect. 3) deals with the application of this new technique to produce vorticity maps of the average supergranular outflows and inflows. Validation of the results will be provided by comparison with another, independent method of obtaining maps of large-scale horizontal flows, namely local correlation tracking (LCT) of granules. LCT provides a higher spatial resolution than time-distance helioseismology, but is restricted to shallow depths, limited by the vertical extension of granules (a few hundred kilometers). In Paper III (Sect.4), the network magnetic field that surrounds the average supergranular outflow is investigated. In Sect. 5, the flow velocity and vorticity profiles associated with the average supergranule are further discussed, and starting points for potential future work are identified. The Appendices include further figures and information that supplement the material from the papers.

In the remainder of this chapter, the tool of helioseismology is introduced as well as the space-borne observatories that provide the high-resolution solar images necessary to conduct this work. For an introduction to LCT, the reader is referred to Paper II, Sect. 3.1.2.

\subsection{Helioseismology}

Helioseismology makes use of solar oscillations (see Sect.1.1.4) to probe the solar interior (for a general discussion of what we have learned from helioseismology, see, e.g., Gough 2013), much like geoseismology uses seismic waves from earthquakes to infer the internal structure of the Earth. Helioseismology can be divided in two branches of methods, global and local helioseismology.

\subsubsection{Global helioseismology}

In global helioseismology, the influence of the physical conditions in the solar interior on normal mode frequencies is studied. The different normal modes are sensitive to different depths and latitudes, which can be expressed by weight functions, the so-called sensi- 
tivity kernels, for a given physical quantity (for example, the sound speed). Knowledge of these kernels (through solar models) and measurements of the mode frequencies can be combined in an inversion problem to infer the specific structure of the physical quantity. This has been done successfully to obtain radial profiles of the sound speed (e.g., Christensen-Dalsgaard et al. 1985) and the rotation rate (Duvall et al. 1984) in the early days of helioseismology.

The sound speed profile has been measured down to the solar core, thus constraining the physical conditions for nuclear fusion. This was an important result with implications even beyond astrophysics: Under the inferred conditions the estimated neutrino production rate in the solar core was much too high to be compatible with neutrino detections on Earth. This problem was resolved later by particle physicists through the discovery of neutrino oscillations (see, e.g., Turck-Chièze \& Couvidat 2011, for a review).

The solar rotation profile was later extended to two dimensions, including the latitudinal direction (e.g., Thompson et al. 1996, Schou et al.|1998), revealing that the latitudinal differential rotation extends through most of the convection zone and that the radiative zone rotates like a solid body. Using almost 20 years of space-based observations, Barekat et al. (2014) recently provided reliable measurements of the radial rotation gradient in the near-surface shear layer up to $60^{\circ}$ latitude.

Another way to study the oscillations of the Sun globally is by looking at diskintegrated lightcurves. The loss of spatial resolution means that information about most modes is lost - only low-degree modes $\ell \lesssim 3$ are retained. However, the disk-integrated lightcurves allow to view the Sun "as a star", since for other stars photometric observations do not provide any spatial resolution. The study of the oscillations of other others, dubbed asteroseismology (see, e.g., Aerts et al. 2010, for an introduction), benefits from the more detailed knowledge of the Sun. In turn, asteroseismology of Sun-like stars helps to put our knowledge of the Sun into perspective.

\subsubsection{Local helioseismology}

In contrast to global helioseismology, local helioseismology can provide three-dimensional maps of flows, sound speed, and other quantities. Instead of using the standing waves (normal modes) of the full Sun, the full field of traveling waves in localized solar patches is considered. A comprehensive overview of local helioseismology methods is given in Gizon et al. (2010) and Gizon \& Birch (2005). For a review on how solar convection can be probed by helioseismology, see Hanasoge et al. (2015).

The term local helioseismology refers to a variety of techniques. The conceptually simplest and oldest technique is the so-called ring-diagram analysis (Hill 1988), which divides the solar disk into small regions and extends the global helioseismology method by analyzing the power spectrum (wave power as a function of frequency $\omega / 2 \pi$ and wavenumber $k$, where $k R_{\odot} \approx \ell$, see, e.g., Fig. A.1 in the Appendix) of each area separately. In each area, $\mathrm{p}$ - and f-mode ridges are visible as rings in cuts of the power spectrum for constant frequency (the power in these cuts is a function of wavenumber and horizontal direction). If there is no background flow, the rings appear as circles with their centers in the origin. If, however, there is a flow in a particular direction, the wave frequencies are Doppler-shifted and the rings are deformed and shifted in the direction of the flow. The magnitude and direction of the flow in a certain depth can be inferred by 
fitting and inversion procedures. The spatial resolution is, however, limited by the size of the regions.

In time-distance helioseismology (Duvall et al. 1993b), the travel times of waves are measured by evaluating the temporal cross-covariance of the filtered Doppler velocity signals between pairs of points (or more complex geometries). The spatial resolution is limited by the wavelength (e.g., about $5 \mathrm{Mm}$ for $\mathrm{f}$ modes at maximum power). The technique of time-distance helioseismology is applied in this thesis and will be explained in more detail in the following chapters (see, e.g., Paper I, Sect. 2.1).

Holography is a method based on wave optics. The idea is that the wavefield at a particular point in a particular depth at a particular time is completely determined by the observed wavefield at the surface (Lindsey \& Braun 1990, Roddier 1975). This technique can also be used to investigate active regions on the far side of the Sun (González Hernández et al. 2007).

In Fourier-Hankel analysis, the wavefield in a surface annulus around a certain point is decomposed into an inward and outward moving component (Braun et al. 1987). This is particularly useful for analyzing the absorption and phase shifts introduced by sunspots (e.g., Crouch et al.2005).

Direct modeling makes use of the fact that the components of the wavefield in Fourier space are uncorrelated for models where spatial and temporal homogeneity is assumed (Woodard 2002). Flows, for example, introduce correlations that can be measured and used as input for an inversion to recover the subsurface flows.

\subsection{Observatories}

\subsubsection{SOHO/MDI}

Until the mid-1990s, local helioseismic observations were conducted from ground-based observatories that had to struggle with day-night cycles, changing weather conditions and image distortions due to Earth's atmosphere. The day-night cycles could be avoided for a few months per year by observing at the South Pole (e.g., Duvall et al. 1993a), but for nearly-continuous, long-term observations a proper space-borne observatory was needed. This was put into action with the launch of the Solar and Heliospheric Observatory (SOHO) (Domingo et al. 1995) in December 1995. SOHO was placed in an orbit at the Lagrangian point $\mathrm{L}_{1}$ between the Sun and the Earth to allow for an unobscured view on the Sun. The satellite contains the Michelson Doppler Imager (MDI) (Scherrer et al. 1995), which obtained full-disk images of the Sun at five wavelengths near the photospheric Ni I $6768 \AA$ absorption line. These so-called filtergrams were combined to obtain photospheric maps of the line-of-sight velocity (Dopplergrams), line-of-sight magnetic field (magnetograms), and continuum intensity. MDI operated during the whole solar activity cycle 23, roughly from one minimum to the next (see Fig. 1.1), until it was turned off in early 2011. The instrument operated in different modes, allowing for full-disk images with a high duty cycle only for about two months per year. 


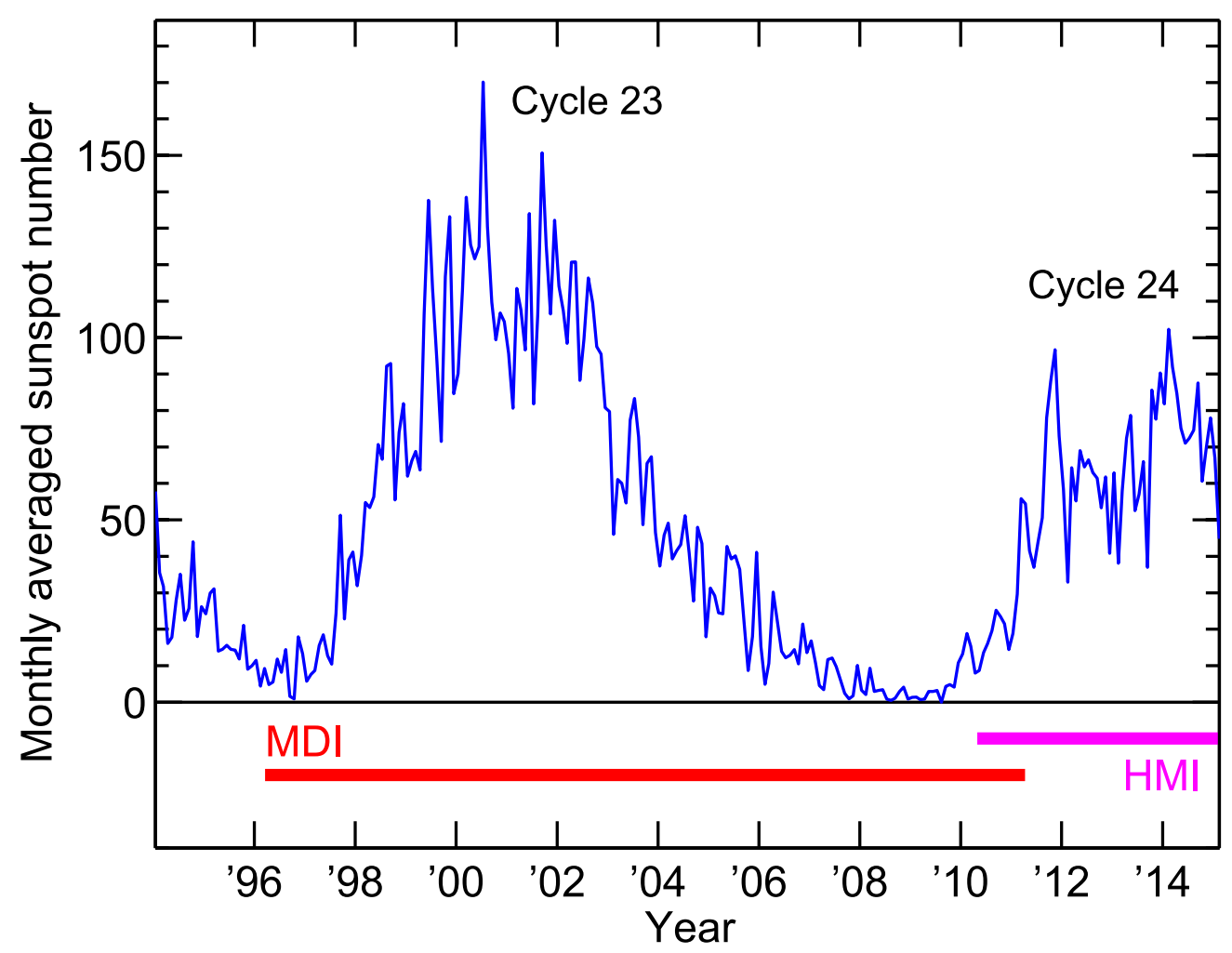

Figure 1.1: Operation period of the SOHO/MDI and SDO/HMI space-borne instruments in relation to the solar cycle, as indicated by the monthly averaged sunspot number. ${ }^{1}$

\subsubsection{SDO/HMI}

The successor of MDI is the Helioseismic and Magnetic Imager (HMI) (Schou et al. 2012, Scherrer et al. 2012) onboard the Solar Dynamics Observatory (SDO) (Pesnell et al. 2012) that was launched in February 2010 and injected into a geosynchronous orbit. HMI can be considered as an improved version of MDI, offering a better spatial sampling $\left(0.348 \mathrm{Mm} \mathrm{px}^{-1}\right.$ versus $1.4 \mathrm{Mm} \mathrm{px}^{-1}$ at disk center) and higher resolution (about $0.7 \mathrm{Mm}$ versus $2.4 \mathrm{Mm}$, Yeo et al. 2014, Korzennik et al.(2004) as well as smaller instrumental systematic errors (e.g., Korzennik et al.|2004, Couvidat et al.|2012b, Liu et al.|2012, Wachter et al. 2012) and an all-year full-disk full-resolution coverage of the Sun. In contrast to MDI, HMI observes at the spectral absorption line Fe I $6173 \AA$, which provides a higher sensitivity to magnetic fields (Norton et al. 2006). Photospheric Dopplergrams, line-ofsight magnetograms, and intensity images are computed from six filtergrams that probe the line core and flanks as well as the nearby continuum (Couvidat et al.2012a). Recently, also full vector magnetograms have been made available (Hoeksema et al. 2014).

MDI was turned off after HMI had started to operate, however only after several months of overlap, during which the two instruments observed in parallel (see time lines

\footnotetext{
${ }^{1}$ The monthly average was tabulated by David Hathaway based on the International Sunspot Number, http://solarscience.msfc.nasa.gov/greenwch/spot_num.txt, see http://solarscience. msfc.nasa.gov/SunspotCycle. shtml for description.
} 
in Fig. 1.1). The temporal overlap and the similar viewing angles of MDI and HMI make it possible to analyze observations for the same solar regions using two different instruments. This can be used, for instance, to confirm measurements from one instrument or to evaluate how sensitive measurements are to instrumental systematics. For this reason, and because the overlap period falls into a phase of comparatively low solar activity, observations from this period are used for the work presented in this thesis.

\subsubsection{Other observatories}

Other noteworthy observatories that produce data products suitable for time-distance helioseismology but are not used in this work include the Solar Optical Telescope (Tsuneta et al. 2008) onboard the Hinode spacecraft (Kosugi et al. 2007) as well as the groundbased Global Oscillation Network Group (GONG) (Harvey et al. 1996) and the Taiwan Oscillation Network (TON) (Chou et al. 1995).

A future space-borne instrument will be the Polarimetric and Helioseismic Imager (PHI) (Solanki et al. 2015) onboard the Solar Orbiter spacecraft. In contrast to SOHO and SDO, Solar Orbiter will leave the near-Earth region and aim for an elliptical orbit around the Sun with a perihelion at about 0.3 astronomical units, close to Mercury's orbit. The spacecraft's orbital plane will be tilted with respect to the ecliptic, enabling for the first time observations of the Sun's poles without suffering from strong foreshortening that usually results from the extremely shallow observation angles. However, time-distance helioseismology will be a challenge due to the elliptical orbit and telemetry restrictions (Löptien et al. 2014a b). Currently, the launch of Solar Orbiter is scheduled for 2018 (Solanki et al. 2015), but the extreme target orbit requires complex maneuvers so that the largest tilt with respect to the ecliptic $\left(22^{\circ}\right)$ will not be reached until 2023. In a possible extended mission phase, the tilt angle might be increased up to $33^{\circ}$ in 2026 . 


\title{
2 Paper I: Time-distance helioseismology: A new averaging scheme for measuring flow vorticity
}

\begin{abstract}
Time-distance helioseismology provides information about vector flows in the near-surface layers of the Sun by measuring wave travel times between points on the solar surface. Specific spatial averages of travel times have been proposed for distinguishing between flows in the east-west and north-south directions and measuring the horizontal divergence of the flows. No specific measurement technique has, however, been developed to measure flow vorticity. Here we propose a new measurement technique tailored to measuring the vertical component of vorticity. Fluid vorticity is a fundamental property of solar convection zone dynamics and of rotating turbulent convection in particular. The method consists of measuring the travel time of waves along a closed contour on the solar surface in order to approximate the circulation of the flow along this contour. Vertical vorticity is related to the difference between clockwise and anti-clockwise travel times. We applied the method to characterize the vortical motions of solar convection using helioseismic data from the Helioseismic and Magnetic Imager onboard the Solar Dynamics Observatory (SDO/HMI) and from the Michelson Doppler Imager onboard the Solar and Heliospheric Observatory (SOHO/MDI). Away from the equator, a clear correlation between vertical vorticity and horizontal divergence is detected. Horizontal outflows are associated with negative vorticity in the northern hemisphere and positive vorticity in the southern hemisphere. The signal is much stronger for HMI than for MDI observations. We characterize the spatial power spectrum of the signal by comparison with a noise model. Vertical vorticity at horizontal wavenumbers below $250 / R_{\odot}$ can be probed with this helioseismic technique. 1$]$
\end{abstract}

\subsection{Introduction}

The Sun exhibits complex flow patterns in the convection zone, such as turbulent convection, differential rotation, and meridional circulation. These flows are important ingredi-

\footnotetext{
${ }^{1}$ This chapter reproduces the article Time-distance helioseismology: A new averaging scheme for measuring flow vorticity by J. Langfellner, L. Gizon, and A. C. Birch, published in Astronomy \& Astrophysics 570, A90 (2014), DOI 10.1051/0004-6361/201424201. Reproduced with permission from Astronomy \& Astrophysics, (c) ESO. Contributions: JL, LG, and ACB designed research. JL performed research, analyzed data, and wrote the paper.
} 
2 Paper I: Time-distance helioseismology: A new averaging scheme for measuring flow vorticity

ents for understanding global solar dynamics and the dynamo responsible for the solar 22-year magnetic cycle (cf. Toomre 2002). Fluid vorticity is a fundamental characteristic of fluid dynamics. The interplay between turbulent convection and rotation can generate cyclonic motions with a net kinetic helicity that depends on solar latitude (Duvall \& Gizon 2000). These motions may convert the toroidal magnetic field into a poloidal field (Parker 1979). Vortices are not confined to convective motions. Hindman et al. (2009) detected that inflows into active regions (Gizon et al. 2001) have a cyclonic component that is presumably caused by solar rotation.

Duvall et al. (1993b) showed that near-surface solar flows can be measured using time-distance helioseismology. The idea is to measure the time it takes for solar waves to travel between two surface locations from the temporal cross-covariance of the observable measured at these locations. Typically, the observable is a series of line-of-sight Doppler velocity images, $\phi(\mathbf{r}, t)$, which has been filtered in the Fourier domain to select particular wave packets. We consider a pair of points $\mathbf{r}_{1}$ and $\mathbf{r}_{2}$ ("point-to-point geometry"). The cross-covariance $C$ at time lag $t$ is

$$
C\left(\mathbf{r}_{1}, \mathbf{r}_{2}, t\right)=\frac{h_{t}}{T} \sum_{i=-N}^{N} \phi\left(\mathbf{r}_{1}, t_{i}\right) \phi\left(\mathbf{r}_{2}, t_{i}+t\right),
$$

where $h_{t}$ is the temporal cadence, $T=(2 N+1) h_{t}$ the observation time, and $t_{i}=i h_{t}$ with $i=-N,-N+1, \ldots, N$ are the times at which the observable is sampled. From the crosscovariance, the travel time can be measured by fitting a wavelet (e. g., Duvall et al. 1997) or a sliding reference cross-covariance (Gizon \& Birch 2004).

Waves are advected by the flow field $\mathbf{v}(\mathbf{r})$, and travel times are sensitive to flows in the vicinity of the ray connecting the points $\mathbf{r}_{1}$ and $\mathbf{r}_{2}$. If the flow has a component in the direction $\mathbf{r}_{2}-\mathbf{r}_{1}$, then the travel time from $\mathbf{r}_{1}$ to $\mathbf{r}_{2}$ (denoted by $\tau^{+}\left(\mathbf{r}_{1}, \mathbf{r}_{2}\right)$ ) is reduced, while the travel time from $\mathbf{r}_{2}$ to $\mathbf{r}_{1}$ (denoted by $\tau^{-}\left(\mathbf{r}_{1}, \mathbf{r}_{2}\right)$ ) is increased.

To obtain a measurement that is particularly sensitive to the horizontal flow divergence $\operatorname{div}_{h}$, travel times are measured between a central point $\mathbf{r}$ and a surrounding annulus with radius $\Delta$ (Duvall et al. 1993b). This "point-to-annulus geometry" is displayed in Fig. 2.1a. The flow divergence is related to the difference between inward and outward travel times.

Duvall et al. (1997) proposed to break the annulus into four quadrants pointing in the east, west, north, and south directions, respectively. Here we remind the reader that the solar convention is that west is in the prograde direction of solar rotation. The travel time measured between $\mathbf{r}$ and the west (or the east) quadrant ("point-to-quadrant geometry") is sensitive to the component of the flow velocity in the west direction, $v_{x}$. In practice, the difference of the quadrants is used. In the same fashion, the north component of the flow velocity, $v_{y}$, can be obtained using the north and south quadrants.

There is no specific measurement geometry, however, that is directly sensitive to the flow vorticity. So far, the vertical component of flow vorticity, $\omega_{z}=\partial_{x} v_{y}-\partial_{y} v_{x}$, has been estimated by taking spatial derivatives of the west-east and north-south travel times (see, e.g., Gizon et al. 2000). Alternatively, one could take the spatial derivatives of inverted flow velocities. We would like though a travel-time measurement that is close to the vorticity before performing any inversion. Furthermore, taking derivatives of noisy quantities (as in both cases above) is a dangerous operation. Thus it is desirable to have a travel-time measurement geometry that is explicitly tailored to measure vorticity and that avoids numerical derivatives. 

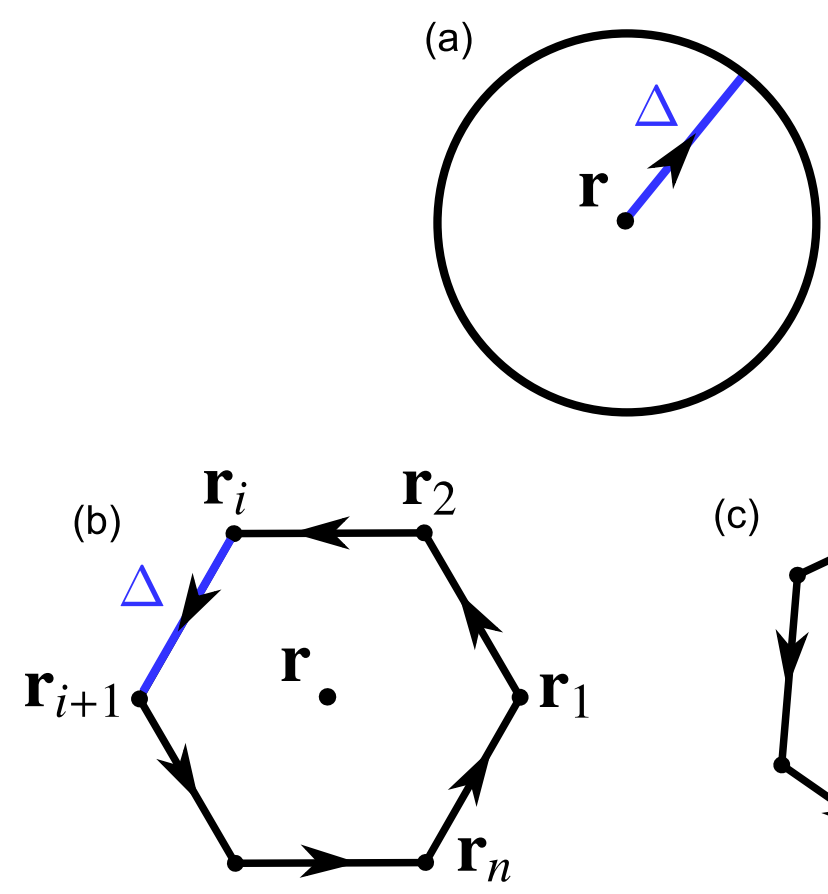

(c)

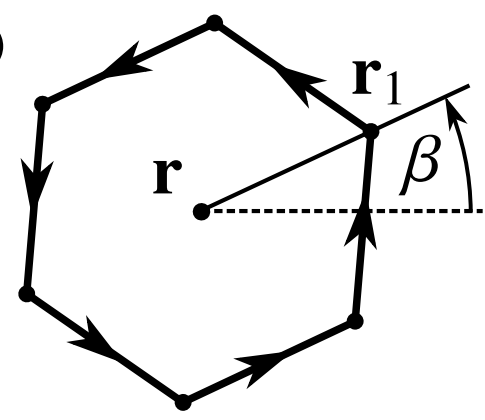

Figure 2.1: Travel-time measurement geometries. a) Measurement geometry sensitive to the horizontal component of the flow divergence. Travel times are measured between a central point $\mathbf{r}$ and the average over a surrounding annulus with radius $\Delta$ as introduced by Duvall et al. (1993b). b) Proposed new measurement geometry sensitive to the vertical component of flow vorticity. Travel times are measured sequentially along neighboring pairs of points $\mathbf{r}_{i}$ and $\mathbf{r}_{i+1}$ located on a closed contour. In this example, $n=6$ points are used, forming a regular hexagon. c) As (b), but the hexagon is rotated by an angle $\beta$ around $\mathbf{r}$.

\subsection{Measuring vortical flows along a closed contour}

In this paper, we implement a measurement technique that is used in ocean acoustic tomography (Munk et al. 1995), where wave travel times are measured along a closed contour $C$. This measurement returns the flow circulation along the contour. The flow circulation is related to the vertical component of vorticity $\omega_{z}$ (averaged over the area $A$ enclosed by the contour) by Stokes' theorem:

$$
\left\langle\omega_{z}\right\rangle_{A}=\frac{1}{A} \int_{A}(\boldsymbol{\nabla} \times \mathbf{v}) \cdot \mathrm{d} \mathbf{A}=\frac{1}{A} \oint_{C} \mathbf{v} \cdot \mathrm{d} \mathbf{l},
$$

where $\mathbf{v}$ is the flow velocity vector on the surface $A$. The vector $\mathrm{dA}$ is normal to the solar surface (upward) and the contour integral runs anti-clockwise with dl tangential to the contour.

\subsubsection{Geometry for anti-clockwise travel times}

We approximate the contour integral as follows. We select $n$ points $\mathbf{r}_{1}, \mathbf{r}_{2}, \ldots, \mathbf{r}_{n}$ along a circular contour $C$ and measure the travel times $\tau^{+}$pairwise in the anti-clockwise direction. Neighboring points are each separated by an equal distance $\Delta$. The points form 
the vertices of a regular polygon (Fig. 2.1 b). Averaging over the $\tau^{+}$measurements yields what we call the "anti-clockwise travel time" $\tau^{\cup}$,

$$
\tau^{\cup}(\mathbf{r}, \Delta, n):=\frac{1}{n} \sum_{i=1}^{n} \tau^{+}\left(\mathbf{r}_{i}, \mathbf{r}_{i+1}\right)
$$

with the notation $\mathbf{r}_{n+1}=\mathbf{r}_{1}$. With this definition, $\tau^{\cup}$ is reduced when there is a flow velocity $v_{\phi}$ tangential to the circle of radius $R=\Delta /[2 \sin (\pi / n)]$ in anti-clockwise direction. In order to provide a simplified description of the relationship between $\tau^{\cup}$ and $\omega_{z}$, we may write the perturbation to the anti-clockwise travel time caused by $v_{\phi}$ as

$$
\delta \tau^{\cup} \approx-\tau_{0} \frac{v_{\phi}}{V_{\text {ref }}} \sim-\frac{\tau_{0} R}{2 V_{\text {ref }}} \omega_{z}
$$

where $\tau_{0}$ is the unperturbed travel time and $V_{\text {ref }}$ is the reference wave speed. This description only provides a rule-of-thumb connection between $\tau^{\cup}$ and $\omega_{z}$. The proper relationship is described by 3D sensitivity kernels (Birch \& Gizon|2007). Additionally, these quantities are functions of all three spatial dimensions, which has not been accounted for.

We note that the distance $\Delta$ between the points must be greater than the wavelength (e. g., about $5 \mathrm{Mm}$ for the $\mathrm{f}$ mode at $3 \mathrm{mHz}$ ), in order to distinguish waves propagating from $\mathbf{r}_{i}$ to $\mathbf{r}_{i+1}$ from waves propagating in the opposite direction. Also there is some freedom in selecting the contour $C$. For example, active regions are often shaped irregularly. To measure the vorticity around active regions, it may be useful to adapt the contour to the shape of the active region.

\subsubsection{Reducing the noise level}

The definition of the anti-clockwise travel times (Eq. (3.3) ) assumes that pairwise travel times can be measured, irrespective of the noise level. This is not a problem in the quiet Sun using the travel-time definition of Gizon \& Birch (2004), which is very robust with respect to noise. However, a wavelet fit to the cross-covariance (as in Duvall et al. 1997) is only possible when the noise is sufficiently low. This is not the case for a single pair of points (see Fig. 2.2 a for an example using f-mode-filtered SDO/HMI data with $\Delta=$ $10 \mathrm{Mm})$.

One option is to average $C$ before performing the wavelet fit. At fixed $\Delta$ and $n$, the measurement polygon can be rotated by angles $\beta$ around $\mathbf{r}$ (Fig. 2.1 c). Since plane waves are only weakly correlated for different propagation directions, taking the average over various angles $\beta$ will lower the noise level. In Fig. 2.2 $\mathrm{c}$, an average over eight angles $\beta$ is shown for $n=6$. Furthermore, $C$ can be averaged over several annulus radii $R$ (several $n$ at fixed distance $\Delta$ ). In Fig. $2.2 \mathrm{~d}$, the cross-covariance is averaged over three different annuli ( $n=4,6$, and 8$)$ and angles $\beta$. An additional $4 \times 4$ averaging over the centers of annuli (Fig. 2.2 e) gives a cross-covariance function that has a sufficiently low level of noise to be analyzed by a wavelet fit. Such averaging procedures are often used for measuring outward-inward travel times. Any spatial averaging must be properly taken into account when travel-time inversions are performed later. 


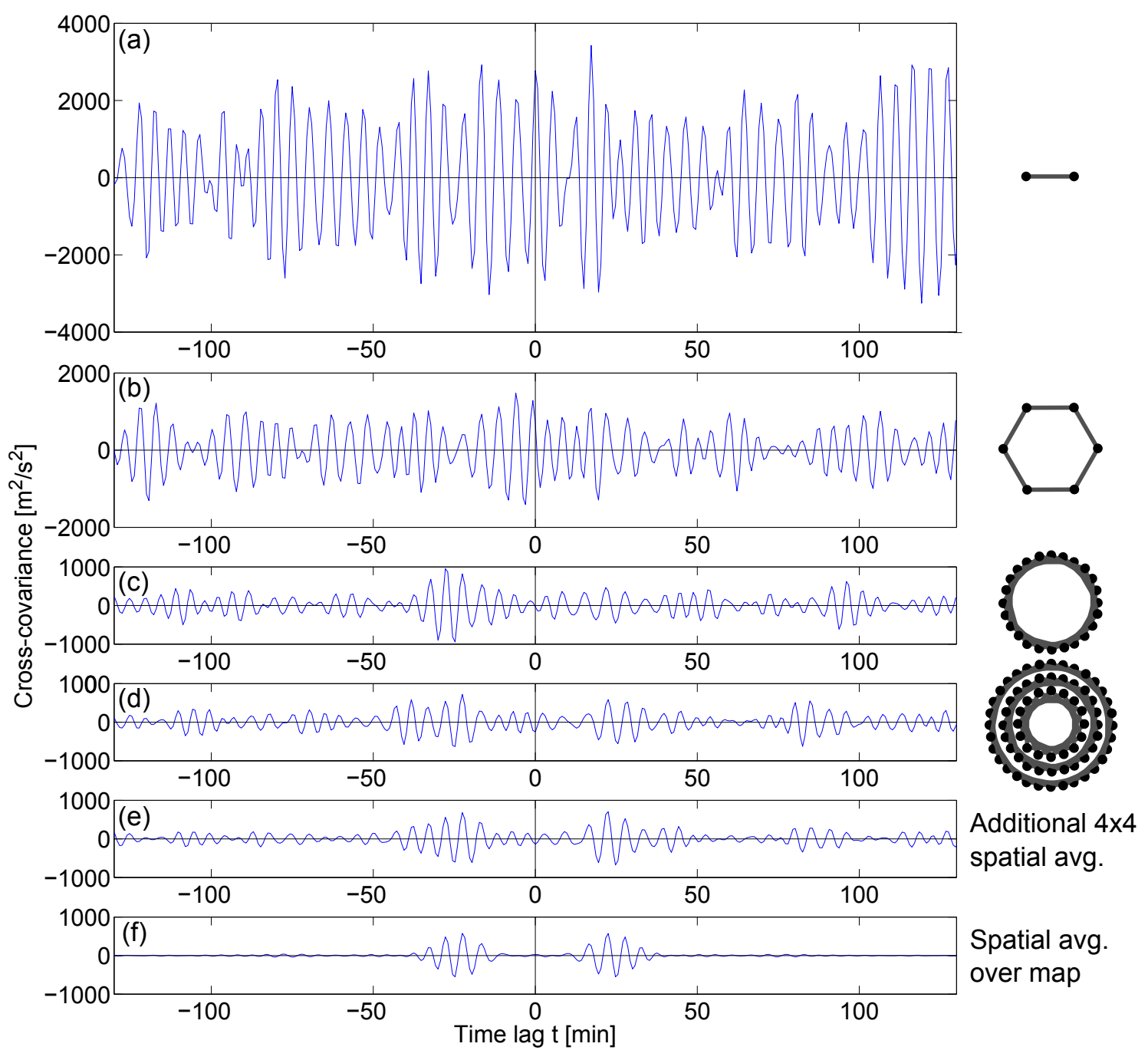

Figure 2.2: F-mode cross-covariance $C$ as a function of time lag for HMI data (see Sect. 2.3.1). All curves except (e) and (f) are for a single center point $\mathbf{r}$ near disk center ( $8 \mathrm{~h}$ averaging, 1 May 2010 dataset). The distance $\Delta$ between consecutive points is $10 \mathrm{Mm}$. a) $C$ for a single pair. b) Averaging over annulus with $n=6$. c) Further averaging over eight angles $\beta$. d) Further averaging over two additional radii: $n=4$ with four angles $\beta$ and $n=8$ with eight angles $\beta$. e) Further averaging over annuli centers $(4 \times 4$ pixels $)$. f) For reference, $n=6$ annulus averaged over an entire map (about $180 \times 180 \mathrm{Mm}^{2}$ ). 


\subsubsection{Decoupling from isotropic wave-speed perturbations}

Since the purpose of $\tau^{\cup}$ is to measure the vorticity $\omega_{z}$, it should ideally not be sensitive to any other solar perturbation. However, a wave-speed perturbation along the contour due to fluctuations in, for instance, temperature or density will also perturb $\tau^{\cup}$. In order to remove travel-time perturbations that are not caused by the flow, we also measure the clockwise travel time

$$
\tau^{\circlearrowright}(\mathbf{r}, \Delta, n):=\frac{1}{n} \sum_{i=0}^{n-1} \tau^{+}\left(\mathbf{r}_{n-i+1}, \mathbf{r}_{n-i}\right) .
$$

For example, the travel times $\tau^{\cup}$ and $\tau^{\cup}$ are affected in the same way by a temperature perturbation. We thus introduce the difference anti-clockwise minus clockwise travel time (denoted with the superscript "ac"),

$$
\tau^{\mathrm{ac}}(\mathbf{r}, \Delta, n):=\tau^{\cup}(\mathbf{r}, \Delta, n)-\tau^{\cup}(\mathbf{r}, \Delta, n) .
$$

Note that $\tau^{\text {ac }} \approx 2 \delta \tau^{\cup}$, where $\delta \tau^{\cup}$ is given by Eq. 2.4. The travel time $\tau^{\text {ac }}$ should be largely independent of perturbations other than a vortical flow. This approach is similar to the one proposed by Duvall et al. (1997) to measure the flow divergence from point-toannulus travel times.

\subsection{Proof of concept using SDO/HMI and SOHO/MDI observations}

In order to test if the proposed averaging scheme is able to measure flow vorticity, we have carried out two simple experiments using $f$ modes. The first experiment (Sect.2.3.3) consists of making maps of $\tau^{\cup}$ using SDO/HMI observations (Schou et al. 2012), computing the spatial power spectrum of these maps, and comparing with the predicted power spectrum of pure realization noise. The second experiment (Sect. 2.3.4) is to look for a correlation between vertical vorticity and horizontal divergence. The sign and the amplitude of this correlation are expected to scale like the local Coriolis number.

\subsubsection{Observations}

We used $112 \times 24$ h series of SDO/HMI line-of-sight velocity images. The Dopplergrams were taken from 1 May to 28 August 2010 when the Sun was relatively quiet. Regions of the size $180 \times 180 \mathrm{Mm}^{2}$ at solar latitudes from $-60^{\circ}$ to $+60^{\circ}$ in steps of $20^{\circ}$ were tracked for one day as they crossed the central meridian. Images were remapped using Postel's projection and tracked at the local surface rotation rate from Snodgrass (1984). The resulting data cubes were cut into three $8 \mathrm{~h}$ datasets. A ridge filter was applied to select $f$ modes.

We also used $56 \times 24 \mathrm{~h}$ series of SOHO/MDI (Scherrer et al. 1995) full-disk lineof-sight velocity images from 8 May through 11 July 2010, thus overlapping with the HMI observations. The MDI data were processed in the same way as for HMI, however the spatial sampling of MDI is lower by a factor of four $\left(2.0 \operatorname{arcsec} \mathrm{px}^{-1}\right.$ instead of 0.5 $\operatorname{arcsec} \mathrm{px}^{-1}$ ) and the temporal cadence is $60 \mathrm{~s}$ instead of $45 \mathrm{~s}$. The spatial resolution is 
given by the instrumental point spread function (PSF), which can be approximated by a Gaussian with a full width at half maximum (FWHM) of about $3.3 \operatorname{arcsec}(2.4 \mathrm{Mm})$ for MDI (Korzennik et al. 2004, 2012) and about $1.0 \operatorname{arcsec}(0.7 \mathrm{Mm})$ for HMI (Yeo et al. 2014).

\subsubsection{Travel-time maps}

From the f-mode-filtered Dopplergrams, we computed the cross-covariance $C$ in Fourier space,

$$
C\left(\mathbf{r}_{1}, \mathbf{r}_{2}, \omega\right)=h_{\omega} \phi_{T}^{*}\left(\mathbf{r}_{1}, \omega\right) \phi_{T}\left(\mathbf{r}_{2}, \omega\right),
$$

where $\omega$ is the angular frequency and $h_{\omega}=2 \pi / T$ is the frequency resolution. The symbol $\phi_{T}$ denotes the Fourier transform of $\phi(\mathbf{r}, t)$ multiplied by the temporal window function. This way of computing $C$ is equivalent to Eq. (2.1), but is much faster. To measure the travel times $\tau^{\cup}$ and $\tau^{\cup}$, we used the linearized definition of travel times as defined by Eq. (3) in Gizon \& Birch (2004) with $W$ given by Eq. (4) in that paper. We obtained the reference cross-covariance $C^{\text {ref }}$ by spatially averaging $C$ over the whole map. We used $\Delta=10 \mathrm{Mm}$ and $n=6$, which corresponds to an annulus radius of $10 \mathrm{Mm}$, and used four different values for $\beta\left(0^{\circ}, 15^{\circ}, 30^{\circ}\right.$, and $\left.45^{\circ}\right)$. We computed $\tau^{\text {ac }}$ as defined in Eq. (2.6). Additionally, we computed outward-inward mean travel-time maps $\left(\tau^{\mathrm{oi}}\right)$ between an annulus radius of $10 \mathrm{Mm}$ and the central point. Again, we used the traveltime definition from Gizon \& Birch (2004).

Figure 2.3 a shows a $\tau^{\mathrm{oi}}$ travel-time map for an example $8 \mathrm{~h}$ dataset at the solar equator. The bluish features of size 20 to $30 \mathrm{Mm}$ are areas of positive divergence. They represent supergranular outflow regions. Conversely, the reddish areas show the supergranular network of converging flows. For the same dataset, a $\tau^{\mathrm{ac}}$ map that was averaged over the four angles $\beta$ is depicted in Fig. 2.3 b. There is no evidence of excess power at the scales of supergranulation.

\subsubsection{Test 1: Evidence of a vorticity signal in $\tau^{\text {ac }}$ as a function of wavenumber}

In order to evaluate the signal-to-noise ratio $(\mathrm{S} / \mathrm{N})$ in the $\tau^{\mathrm{ac}}$ maps, we compare the spatial power of the travel-time maps with a noise model. For the noise model, we use the recipe of Gizon \& Birch (2004) to construct artificial datasets as follows. In 3D Fourier space the observable is modeled by a Gaussian complex random variable with zero mean and variance given by the expected power spectrum (estimated from the observations). In the noise model, wavenumbers and frequencies are uncorrelated to exclude wave scattering by flows and heterogeneities (signal). The expectation value of the power spectrum is chosen to match that of HMI observations. A detailed study and a validation of the noise model is provided by Fournier et al. (2014).

For each HMI dataset, one realization of the noise model was generated, based on the corresponding power spectrum. From these noise datasets, we computed $\tau^{\mathrm{oi}}$ and $\tau^{\mathrm{ac}}$ travel-time maps in the same manner as for the HMI observations (cf. Sect. 2.3.2).

The $\tau^{\mathrm{oi}}$ power spectrum averaged over azimuth and all datasets is shown in Fig. 2.3c. There is signal above noise level for $k R_{\odot}<500$, with a maximum $\mathrm{S} / \mathrm{N}$ of 50 at $k R_{\odot}=120$. 
2 Paper I: Time-distance helioseismology: A new averaging scheme for measuring flow vorticity
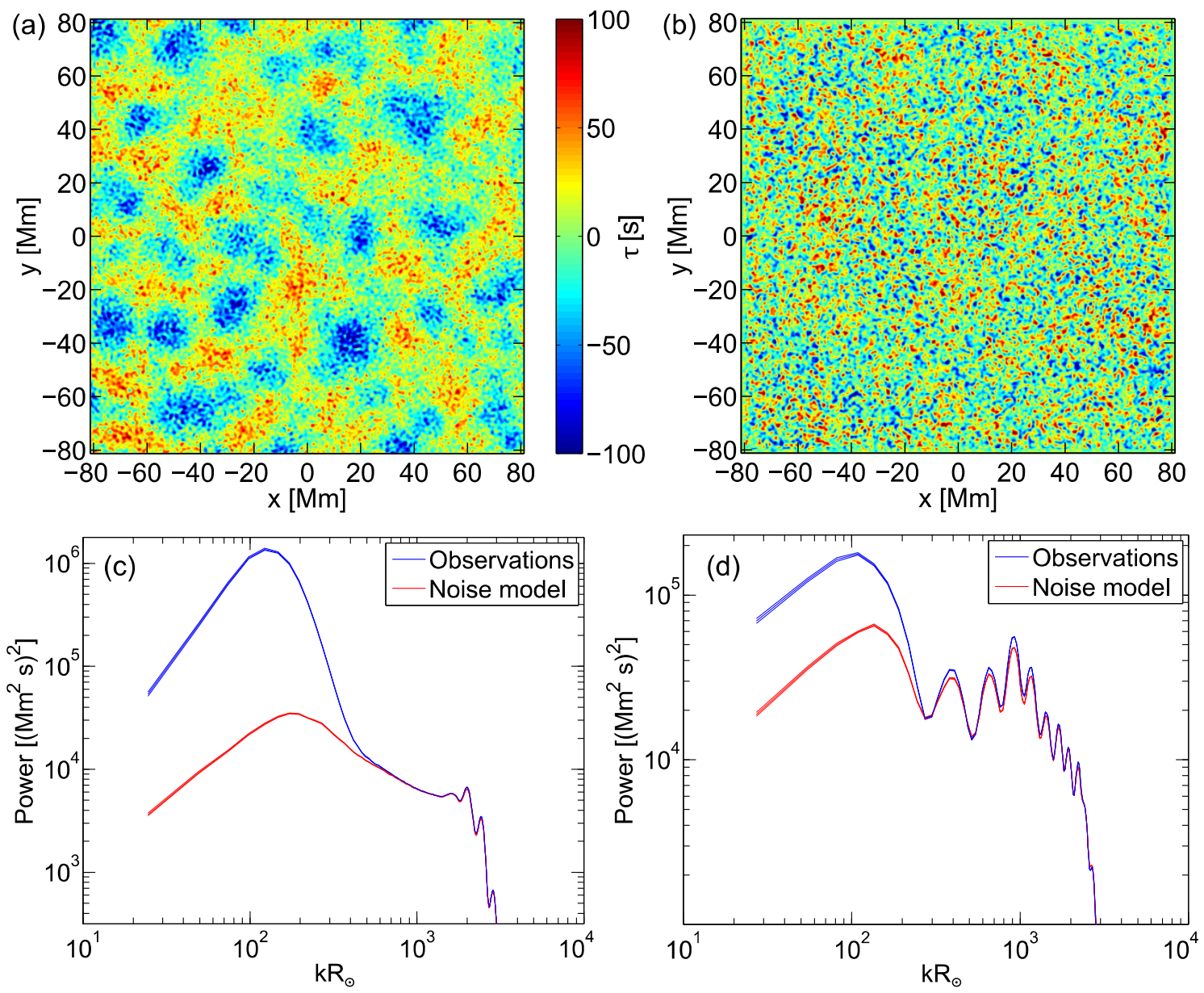

Figure 2.3: a) Example HMI $\tau^{\text {oi }}$ travel-time map for one f-mode-filtered $8 \mathrm{~h}$ dataset centered at the solar equator and annulus radius $\Delta=10 \mathrm{Mm}$. The coordinate $x$ is in the west direction and $y$ in the north direction. The travel times have been measured using the linearized definition in Gizon \& Birch (2004). The color scale has been truncated to increase the contrast. The minimum and maximum values are $-152.4 \mathrm{~s}$ and $117.3 \mathrm{~s}$. b) Example $\tau^{\text {ac }}$ travel-time map for the same dataset as in (a) with $\Delta=10 \mathrm{Mm}$ and $n=6$, averaged over the four angles $\beta$. The minimum and maximum values are $-149.7 \mathrm{~s}$ and $144.8 \mathrm{~s}$. c) Power spectrum of $\tau^{\mathrm{oi}}$ for both HMI data and noise model (Gizon \& Birch 2004), averaged over azimuth and $336 \times 8 \mathrm{~h}$ datasets (size about $180 \times 180 \mathrm{Mm}^{2}$ ) centered at the solar equator, plotted versus the product of horizontal wavenumber $k$ and solar radius $R_{\odot}$. The thickness of the lines denotes the $1 \sigma$ error. d) Power spectrum of $\tau^{\text {ac }}$ for both HMI data and noise model, averaged over azimuth and $336 \times 8$ h datasets. The $\tau^{\text {ac }}$ maps were averaged over four angles $\beta$ before computing the power. 
This is the well-known supergranulation peak. For high wavenumbers $\left(k R_{\odot}>500\right)$, the travel-time maps are dominated by noise. The contribution from convection features that are much shorter lived than the observation time of $8 \mathrm{~h}$ is very small. At $k R_{\odot} \sim 2000$ there is a cut-off in power corresponding to a wavelength of $2.5 \mathrm{Mm}$ (half the f-mode wavelength at $3 \mathrm{mHz}$ ). For MDI (not shown here), the $\mathrm{S} / \mathrm{N}$ at the supergranulation peak is about 16 and the $\mathrm{S} / \mathrm{N}$ vanishes at $k R_{\odot}=400$.

For $\tau^{\text {ac }}$, we averaged the maps over four angles $\beta$, computed the power spectrum for each resulting map and averaged the power spectra over azimuth and all datasets. The result for HMI is shown in Fig. 2.3 d. The power for the HMI travel times significantly exceeds that of the noise model for $k R_{\odot}<250$, with a S/N increasing toward larger scales $\left(\mathrm{S} / \mathrm{N}\right.$ about 1.5 at $\left.k R_{\odot}=120\right)$ and reaching a $\mathrm{S} / \mathrm{N}$ of about 2.6 for $k R_{\odot}=25$. This is qualitatively different than for the $\tau^{\mathrm{oi}}$ power. Again, there is a power cut-off at $k R_{\odot} \sim$ 2000. In the MDI case, the $\mathrm{S} / \mathrm{N}$ is similar but lower than for the HMI data $(\mathrm{S} / \mathrm{N}=0.7$ at $k R_{\odot}=120, \mathrm{~S} / \mathrm{N}=1.3$ at $\left.k R_{\odot}=25\right)$.

For the results presented in Fig. 2.3 d as much as four months of HMI data were averaged. What is the minimum number of days of observations needed to achieve a clear detection of the vorticity signal in the $\tau^{\text {ac }}$ maps (180 Mm on the side)? By clear detection we mean that, at fixed wavenumber, the power in the observed travel times and the power in the noise model are separated by at least $2 \times 3 \sigma$. This requirement is somewhat arbitrary but safe. Figure 2.4 a shows the spatial power of $\tau^{\text {ac }}$ at $k R_{\odot}=109$ as a function of observation duration, for $\tau^{\text {ac }}$ maps averaged over four angles $\beta$. Overplotted are the $3 \sigma$ error estimates (filled areas). Since the data cubes are not correlated from one day to the next (different longitudes), the variance of the noise decreases like 1 over the number of days. The criterion for clear signal detection is fulfilled after two days of observations.

This detection is also a function of the number of angles $\beta$ over which the contours are rotated and averaged. In Fig. 2.4 b, the variance of the $\tau^{\text {ac }}$ travel times is plotted versus this number, $n_{\beta}$. For $\Delta=10 \mathrm{Mm}$, we find that the variance decreases almost like $1 / n_{\beta}$ for small $n_{\beta}$ and reaches a plateau for $n_{\beta}>8$. This is because the $\tau^{\text {ac }}$ measurements are highly correlated for small rotation angles. In this case, $n_{\beta}=4$ is a good compromise between efficient data use and computation time.

\subsubsection{Test 2: Effect of rotation on vorticity in supergranules}

Here we compute $\left\langle\tau^{\mathrm{oi}} \tau^{\mathrm{ac}}\right\rangle$, where the angle brackets denote an average over the solar surface and over all datasets. Since $\tau^{\mathrm{oi}} \propto-\operatorname{div}_{h}$ and $\tau^{\mathrm{ac}} \propto-\omega_{z}$, the product $\left\langle\tau^{\mathrm{oi}} \tau^{\mathrm{ac}}\right\rangle$ serves as a proxy for $\left\langle\operatorname{div}_{h} \omega_{z}\right\rangle$, which is a component of the kinetic helicity and is sensitive to the effect of the Coriolis force on convection (e. g., Zeldovich et al. 1990, Rüdiger et al. 1999).

The latitudinal dependencies of $\left\langle\tau^{\mathrm{oi}} \tau^{\cup}\right\rangle$ and $\left\langle\tau^{\mathrm{oi}} \tau^{\cup}\right\rangle$ for HMI data are plotted in Fig. $2.5 \mathrm{a}$. Note that we filtered the travel-time maps spatially by removing the power for $k R_{\odot}>300$ since there is no significant signal in $\tau^{\mathrm{ac}}$ at high wavenumbers. $\left\langle\tau^{\mathrm{oi}} \tau^{\cup}\right\rangle$ is negative in the northern hemisphere and positive in the southern hemisphere. For $\left\langle\tau^{\mathrm{oi}} \tau^{\mathcal{}}\right\rangle$ the pattern is reversed. At the equator, both quantities have a low positive value of $2 \mathrm{~s}^{2}$, which is probably due to wave-speed perturbations associated with the magnetic network.

In Fig. $2.5 \mathrm{p},\left\langle\tau^{\mathrm{oi}} \tau^{\mathrm{ac}}\right\rangle$ is plotted versus solar latitude for both HMI and MDI data. This allows to compare the sensitivity of both instruments. Additionally, $\left\langle\tau^{\mathrm{oi}} \tau^{\mathrm{ac}}\right\rangle$ was com- 

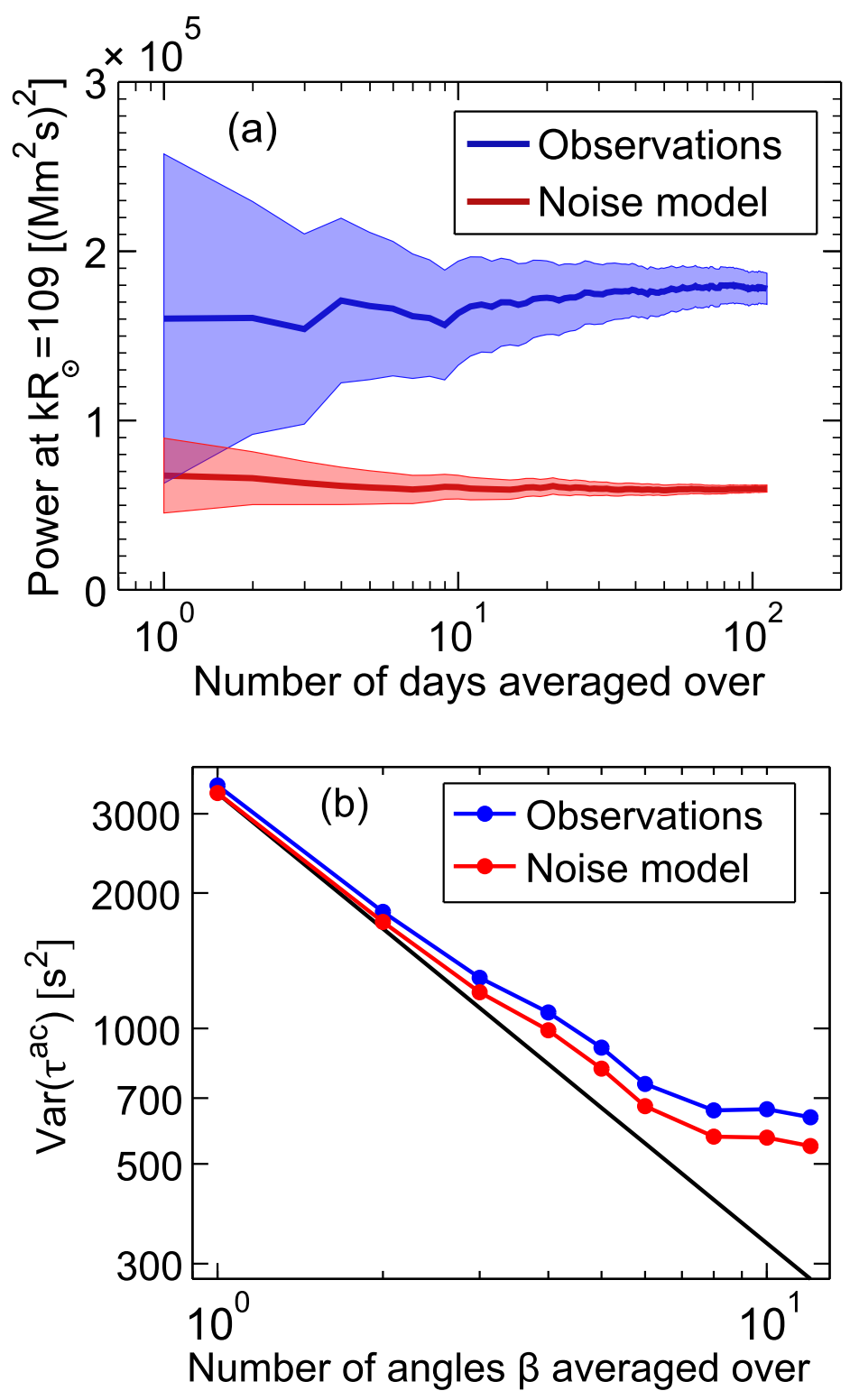

Figure 2.4: a) Azimuthally averaged power of HMI and noise-model $\tau^{\text {ac }}$ maps (average maps over four angles $\beta$ ) at $k R_{\odot}=109$, as a function of days of data averaged over (the same data as in Fig. 2.3 were used). The power was averaged over three $8 \mathrm{~h}$ datasets per day. The filled areas denote the $3 \sigma$ error estimates. b) Variance of $\tau^{\text {ac }}$ measurements computed from both HMI and noise-model f-mode-filtered data at the solar equator. The observation time of the datasets is $8 \mathrm{~h}$ and their spatial size is about $180 \times 180 \mathrm{Mm}^{2}$. The variance is shown as a function of the number of angles $\beta$ over which the data is averaged. For reference, the black line shows $\operatorname{Var}\left(\tau^{\mathrm{ac}}\right) \propto 1 / n_{\beta}$. 

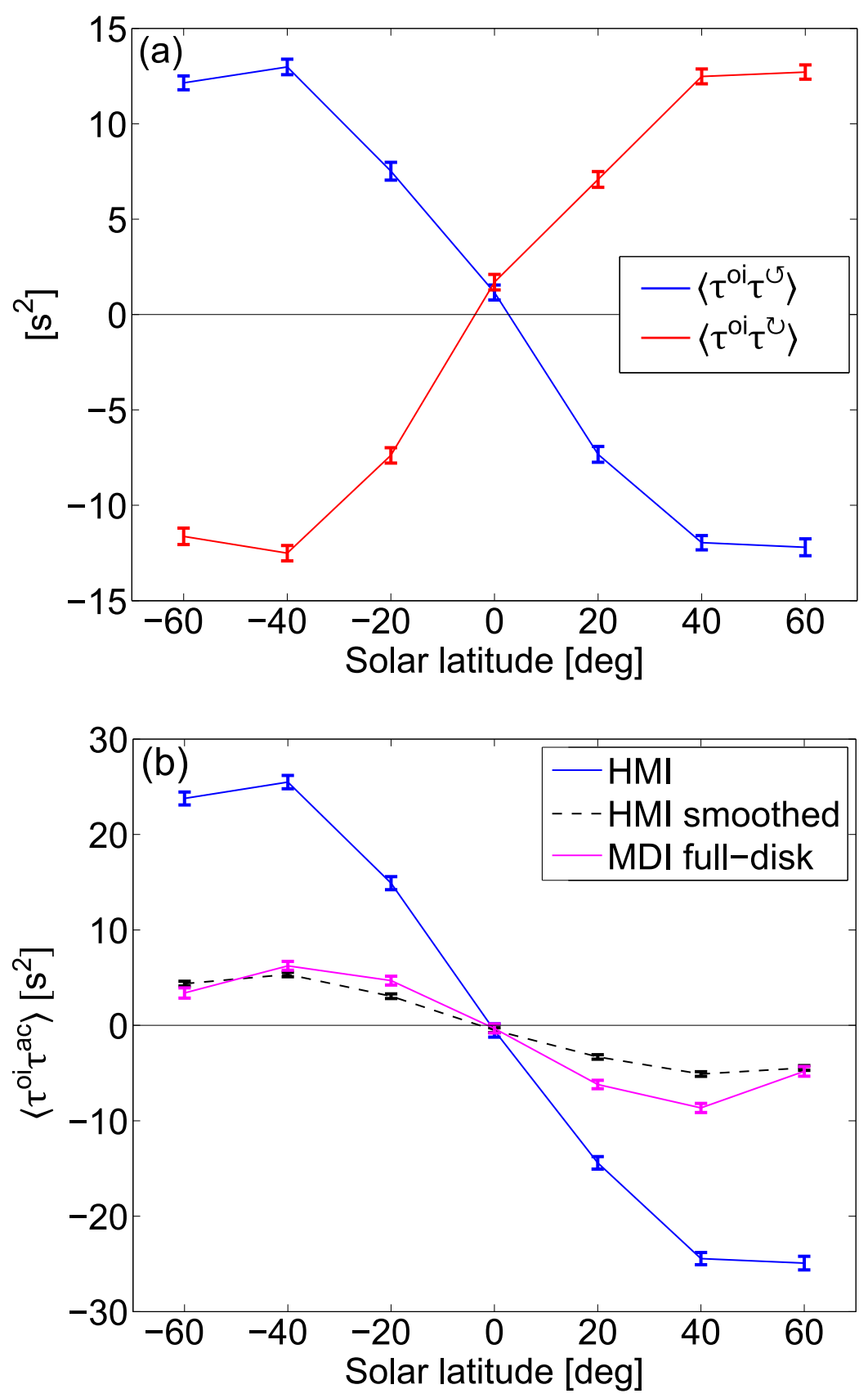

Figure 2.5: a) Horizontal averages $\left\langle\tau^{\mathrm{oi}} \tau^{\cup}\right\rangle$ and $\left\langle\tau^{\mathrm{oi}} \tau^{\cup}\right\rangle$ for f-mode-filtered HMI observations as functions of solar latitude, averaged over $336 \times 8 \mathrm{~h}$ datasets of about $180 \times 180 \mathrm{Mm}^{2}$ and four angles $\beta$. b) Horizontal average $\left\langle\tau^{\mathrm{oi}} \tau^{\mathrm{ac}}\right\rangle$ for different data: HMI at full resolution $\left(0.5 \operatorname{arcsec} \mathrm{px}^{-1}\right)$, MDI full-disk data $\left(2.0 \operatorname{arcsec} \mathrm{px}^{-1}\right)$, and HMI data spatially averaged over $4 \times 4$ px (after remapping) and convolved with a Gaussian with $2.4 \mathrm{Mm}$ FWHM to match the MDI sampling and PSF. 
2 Paper I: Time-distance helioseismology: A new averaging scheme for measuring flow vorticity

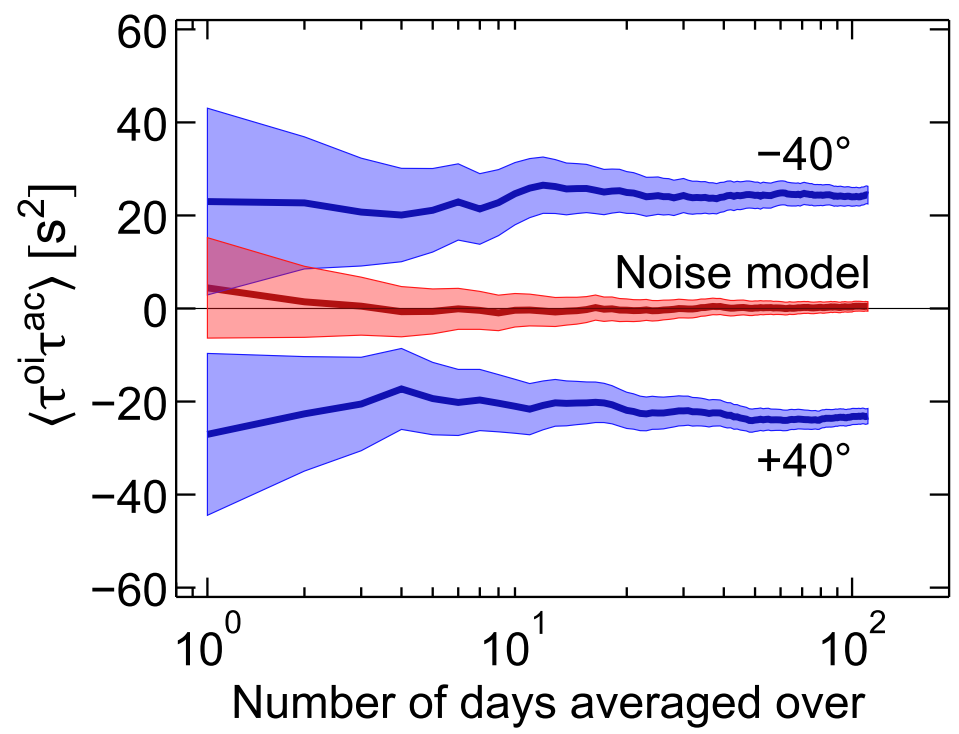

Figure 2.6: Product $\left\langle\tau^{\mathrm{oi}} \tau^{\mathrm{ac}}\right\rangle$ as a function of days of data averaged over, for f-mode-filtered HMI observations at $-40^{\circ}$ and $40^{\circ}$ latitude as well as $\tau^{\text {ac }}$ computed for the noise model. The filled areas denote the $3 \sigma$ estimates. All data have been averaged over space (about $180 \times 180 \mathrm{Mm}^{2}$ ), four angles $\beta$ and three $8 \mathrm{~h}$ datasets per day.

puted from HMI Dopplergrams that were binned over $4 \times 4$ pixels and convolved with a Gaussian with 2.4 Mm FWHM (black curve) such that the spatial resolution matches approximately the resolution of MDI full-disk data. For both instruments, $\left\langle\tau^{\mathrm{oi}} \tau^{\mathrm{ac}}\right\rangle$ is negative in the northern hemisphere and positive in the southern hemisphere. As expected, $\left\langle\tau^{\mathrm{oi}} \tau^{\mathrm{ac}}\right\rangle$ is consistent with zero at the equator (no Coriolis force acts on horizontal flows). Our measurements are qualitatively consistent with the $\left\langle\operatorname{div}_{h} \omega_{z}\right\rangle$ estimates by Duvall $\&$ Gizon (2000) and Gizon et al. (2003).

Away from the equator, we see that the amplitude of $\left\langle\tau^{\mathrm{oi}} \tau^{\mathrm{ac}}\right\rangle$ is higher for HMI than for MDI by a factor of five. This means HMI has a much higher sensitivity to $\left\langle\tau^{\mathrm{oi}} \tau^{\mathrm{ac}}\right\rangle$ than MDI. Only when the HMI Dopplergrams are degraded to match the MDI sampling and PSF, a good agreement of the curves is achieved. Hence the difference in sensitivity to vertical vorticity between HMI and MDI is directly related to the spatial resolution of the instruments.

As in Sect. 2.3.3, we investigate how much data is needed for a clear non-zero detection $\left(3 \sigma\right.$ level) of $\left\langle\tau^{\mathrm{oi}} \tau^{\mathrm{ac}}\right\rangle$ away from the equator. Figure 2.6 shows $\left\langle\tau^{\mathrm{oi}} \tau^{\mathrm{ac}}\right\rangle$ versus the duration of the HMI observations at solar latitudes $\pm 40^{\circ}$. Again the $3 \sigma$ error estimates are plotted as shaded areas. The curves for observations at $40^{\circ}$ and $-40^{\circ}$ latitude do not overlap after averaging over one or more days of data. This means a difference in $\left\langle\tau^{\mathrm{oi}} \tau^{\mathrm{ac}}\right\rangle$ between the hemispheres can be detected at the $3 \sigma$ level after one day of averaging. To distinguish between $40^{\circ}$ observations and the noise-model data takes roughly three days of data. Note that these results are only valid for the given map size of about $180 \times 180 \mathrm{Mm}^{2}$. This corresponds to averaging over roughly 40 supergranules per day. 


\subsection{Conclusion}

We have presented a new averaging scheme for time-distance helioseismology, which has direct sensitivity to the vertical component of the flow vorticity. The anti-clockwise minus clockwise HMI travel-time maps for f modes show power above the noise level. Unlike the divergence signal, the vorticity signal does not peak at supergranular scales but increases continuously toward larger spatial scales. Furthermore, the latitudinal dependence of the correlation between the vorticity and the divergence signals is consistent with the effect of the Coriolis force on turbulent convection. We find that HMI has a much higher sensitivity to this correlation than MDI.

\section{Acknowledgements}

We acknowledge research funding by Deutsche Forschungsgemeinschaft (DFG) under grant SFB 963/1 "Astrophysical flow instabilities and turbulence" (Project A1). The HMI data used are courtesy of NASA/SDO and the HMI science team. The data were processed at the German Data Center for SDO (GDC-SDO), funded by the German Aerospace Center (DLR). We are grateful to C. Lindsey, H. Schunker and R. Burston for providing help with tracking and mapping. We acknowledge the workflow management system Pegasus (funded by The National Science Foundation under OCI SI2-SSI program grant \#1148515 and the OCI SDCI program grant \#0722019). 



\title{
3 Paper II: Spatially resolved vertical vorticity in solar supergranulation using helioseismology and local correlation tracking
}

\begin{abstract}
Flow vorticity is a fundamental property of turbulent convection in rotating systems. Solar supergranules exhibit a preferred sense of rotation, which depends on the hemisphere. This is due to the Coriolis force acting on the diverging horizontal flows. We aim to spatially resolve the vertical flow vorticity of the average supergranule at different latitudes, both for outflow and inflow regions. To measure the vertical vorticity, we use two independent techniques: time-distance helioseismology (TD) and local correlation tracking of granules in intensity images (LCT) using data from the Helioseismic and Magnetic Imager (HMI) on board the Solar Dynamics Observatory (SDO). Both maps are corrected for center-to-limb systematic errors. We find that $8 \mathrm{~h}$ TD and LCT maps of vertical vorticity are highly correlated at large spatial scales. Associated with the average supergranule outflow, we find tangential (vortical) flows that reach about $10 \mathrm{~m} \mathrm{~s}^{-1}$ in the clockwise direction at $40^{\circ}$ latitude. In average inflow regions, the tangential flow reaches the same magnitude, but in the anticlockwise direction. These tangential velocities are much smaller than the radial (diverging) flow component $\left(300 \mathrm{~m} \mathrm{~s}^{-1}\right.$ for the average outflow and $200 \mathrm{~m} \mathrm{~s}^{-1}$ for the average inflow). The results for TD and LCT as measured from HMI are in excellent agreement for latitudes between $-60^{\circ}$ and $60^{\circ}$. From HMI LCT, we measure the vorticity peak of the average supergranule to have a full width at half maximum of about $13 \mathrm{Mm}$ for outflows and $8 \mathrm{Mm}$ for inflows. This is larger than the spatial resolution of the LCT measurements (about $3 \mathrm{Mm}$ ). On the other hand, the vorticity peak in outflows is about half the value measured at inflows (e.g. $4 \times 10^{-6} \mathrm{~s}^{-1}$ clockwise compared to $8 \times 10^{-6} \mathrm{~s}^{-1}$ anticlockwise at $40^{\circ}$ latitude). Results from the Michelson Doppler Imager (MDI) on board the Solar and Heliospheric Observatory (SOHO) obtained in 2010 are biased compared to the HMI/SDO results for the same period. 1 ]
\end{abstract}

\footnotetext{
${ }^{1}$ This chapter reproduces the article Spatially resolved vertical vorticity in solar supergranulation using helioseismology and local correlation tracking by J. Langfellner, L. Gizon, and A. C. Birch, published in Astronomy \& Astrophysics 581, A67 (2015), DOI 10.1051/0004-6361/201526024. Reproduced with permission from Astronomy \& Astrophysics, (c) ESO. Contributions: JL, LG, and ACB designed research. $\mathrm{JL}$ performed research, analyzed data, and wrote the paper.
} 
3 Paper II: Spatially resolved vertical vorticity in solar supergranulation using helioseismology and local correlation tracking

\subsection{Introduction}

Duvall \& Gizon (2000) and Gizon et al. (2003) revealed that supergranules (see Rieutord \& Rincon 2010, for a review) possess a statistically preferred sense of rotation that depends on solar latitude. In the northern hemisphere, supergranules tend to rotate clockwise, in the southern hemisphere anticlockwise. This is due to the Coriolis force acting on the divergent horizontal flows of supergranules. For supergranulation (lifetime $>1$ day), the Coriolis number is close to unity (see Gizon et al. 2010). As a consequence, the vorticity induced by the Coriolis force should be measurable by averaging the vorticity of many realizations of supergranules at a particular latitude.

For single realizations, Attie et al. (2009) detected strong vortices associated with supergranular inflow regions by applying a technique called balltracking. Komm et al. (2007) presented maps of vortical flows in quiet Sun convection using helioseismic ringdiagram analysis. With the same technique, Hindman et al. (2009) resolved the circular flow component associated with inflows into active regions; however, the spatial structure of such vortical flows has not yet been studied for many realizations. Knowledge of the flow structure of the average supergranule will help constrain models and simulations of turbulent convection that take rotation into account.

Here, we aim to spatially resolve the vertical component of flow vorticity associated with the average supergranule. We investigate both outflows from supergranule centers and inflows into the supergranular network. To measure the flow divergence and vorticity, we use two independent techniques: time-distance helioseismology (TD) and local correlation tracking (LCT) of granules. We use the TD method from Langfellner et al. (2014), where a measurement geometry that is particularly sensitive to the vertical component of flow vorticity was defined.

\subsubsection{Time-distance helioseismology}

Time-distance helioseismology makes use of waves travelling through the Sun (Duvall et al. 1993b). A wave travelling from the surface point $\mathbf{r}_{1}$ through the solar interior to another surface point $\mathbf{r}_{2}$ is sensitive to local physical conditions (e.g., the wave speed or density). A flow in the direction $\mathbf{r}_{2}-\mathbf{r}_{1}$ will increase the wave speed, thus reducing the travel time $\tau^{+}$from $\mathbf{r}_{1}$ to $\mathbf{r}_{2}$. A flow in the opposite direction will result in a longer travel time. The travel time is measured from the temporal cross-covariance, labeled $C$, of the observable $\phi$ obtained at the points $\mathbf{r}_{1}$ and $\mathbf{r}_{2}$ :

$$
C\left(\mathbf{r}_{1}, \mathbf{r}_{2}, t\right)=\frac{h_{t}}{T} \sum_{i=-N}^{N} \phi\left(\mathbf{r}_{1}, t_{i}\right) \phi\left(\mathbf{r}_{2}, t_{i}+t\right),
$$

where $h_{t}$ is the temporal cadence, $T=2(N+1) h_{t}$ is the observation time, and $t_{i}=$ $(-N,-N+1, \ldots, N) h_{t}$ are the times when $\phi$ is sampled. Typically, the observable $\phi$ is the Doppler line-of-sight velocity component.

The travel time can be obtained from $C$ by fitting a wavelet (Duvall et al. 1997) or by comparison with a reference cross-covariance and application of an appropriate weight function (Gizon \& Birch 2004). To distinguish the flow signal in the travel time from other perturbations (e.g., local sound speed changes), we use the travel-time difference

$$
\tau^{\mathrm{diff}}\left(\mathbf{r}_{1}, \mathbf{r}_{2}\right)=\tau^{+}\left(\mathbf{r}_{1}, \mathbf{r}_{2}\right)-\tau^{+}\left(\mathbf{r}_{2}, \mathbf{r}_{1}\right)
$$


Travel times that are especially sensitive to the horizontal flow divergence can be obtained by replacing $\mathbf{r}_{2}$ with an annulus around $\mathbf{r}_{1}$ (see Fig. 3.1 1). Averaging $\phi$ over the annulus yields the "outward-inward" travel time $\tau^{\text {oi }}$ (Duvall et al. 1996). To obtain travel times that measure the vertical component of the flow vorticity, we average $\tau^{\text {diff }}$ components along a closed contour in the anticlockwise direction (Langfellner et al. 2014). We choose the contour to be a regular polygon with $n$ points and edge length $\Delta$ in order to approximate an annulus (see Fig. $3.1 \mathrm{~b}$ ). The mean over the $\tau^{\text {diff }}$ components gives the vorticity-sensitive $\tau^{\text {ac }}$ travel time,

$$
\tau^{\mathrm{ac}}(\mathbf{r}, \Delta, n):=\frac{1}{n} \sum_{i=1}^{n} \tau^{\mathrm{diff}}\left(\mathbf{r}_{i}, \mathbf{r}_{i+1}\right),
$$

where we use the notation $\mathbf{r}_{n+1}=\mathbf{r}_{1}$.

\subsubsection{Local correlation tracking}

Local correlation tracking measures how structures in solar images are advected by background flows. For the tracer, it is common to use solar granulation observed in photospheric intensity images (November \& Simon 1988). The general procedure is as follows. Pairs of images are selected that observe the same granules but are separated by a time $\Delta t$. This time separation must be small compared to the lifetime of granules, i.e., $\Delta t \ll 10$ min. To obtain spatially resolved velocity maps, an output map grid is defined. For each grid point, subsets of the intensity images that are centered around the grid point are selected by applying a spatial window and multiplied by a Gaussian with a full width at half maximum (FWHM), typically of a few megameters. The subsets are then crosscorrelated in the two spatial image dimensions $x$ and $y$. The peak position $(\Delta x, \Delta y)$ of the cross-correlation yields the spatial shift. Since the measured shift is usually only a small fraction of a pixel, it must be obtained using an appropriate fitting procedure. Finally, the velocity components in the $x$ and $y$ directions are given by $v_{x}=\Delta x / \Delta t$ and $v_{y}=\Delta y / \Delta t$.

The LCT method has proven valuable when measuring flow patterns in the Sun. For instance, Brandt et al. (1988) and Simon et al. (1989) observed single vortex flows at granulation scale. Hathaway et al. (2013) detected giant convection cells with LCT of supergranules in Doppler velocity images. For a comparison of different LCT techniques, see Welsch et al. (2007).

\subsection{Observations and data processing}

The basis for our measurements of wave travel times and flow velocities from local correlation tracking are two independent observables. We use Doppler velocity images for the TD and intensity images for the LCT. Both observables are measured for the full solar disk by the Helioseismic and Magnetic Imager (HMI) on board the Solar Dynamics Observatory (SDO) (Schou et al.2012) and are available for the same periods of time. This allows a direct comparison of the two methods for looking at "the same Sun" but utilizing independent data.

We used 112 days of both SDO/HMI Dopplergrams and intensity images in the period from 1 May through 28 August 2010. Patches of approximate size $180 \times 180 \mathrm{Mm}^{2}$ were 
(a)

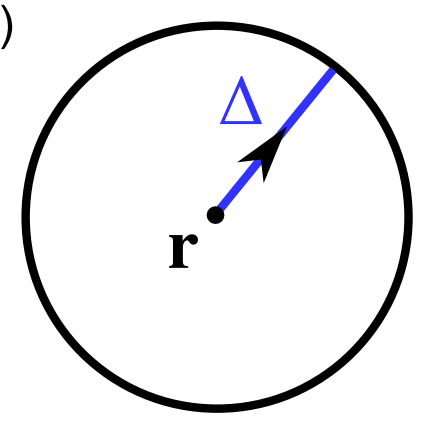

(c)

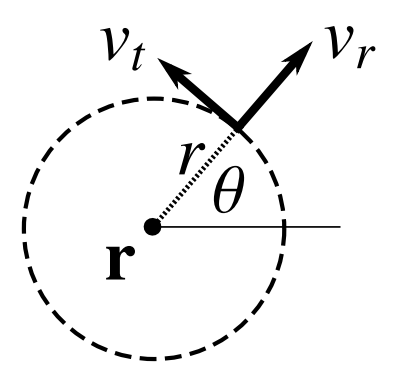

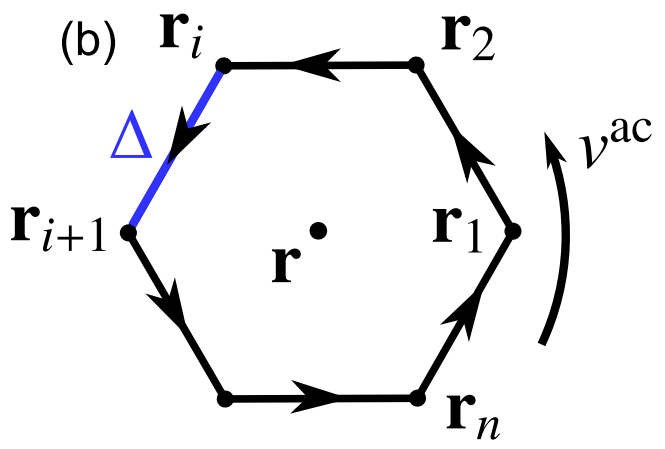

(d)

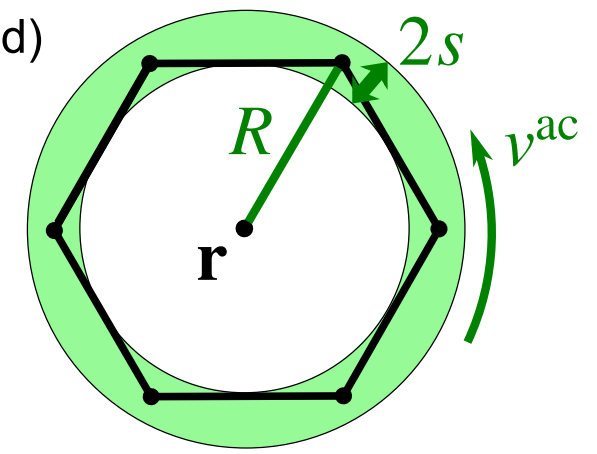

Figure 3.1: Measurement geometries for divergence- and vorticity-sensitive travel times and LCT velocities. a) The divergence-sensitive travel time $\tau^{\mathrm{oi}}$ is obtained by measuring the travel-time difference between a central point $\mathbf{r}$ and a surrounding annulus of radius $\Delta$. b) The vorticity-sensitive travel time $\tau^{\text {ac }}$ is obtained by measuring the travel-time differences $\tau^{\text {diff }}$ between adjacent points along a regular polygon surrounding $\mathbf{r}$. The polygon consists of $n$ points and has edges of length $\Delta$. The points are situated on a circle of radius $R=\Delta /[2 \sin (\pi / n)]$. The travel time $\tau^{\text {ac }}$ is the average over the components $\tau^{\text {diff }}$ (see Eq. (3.3)). We obtain circulation velocities $v^{\text {ac }}$ from multiplying $\tau^{\text {ac }}$ by a calibration factor. c) From LCT, we obtain the horizontal velocity components $v_{x}$ and $v_{y}$. Given a reference point $\mathbf{r}$, the LCT velocities can be expressed in 2D polar coordinates $(r, \theta)$ by the outward pointing radial velocity component $v_{r}$ and the anticlockwise pointing tangential velocity component $v_{t}$. d) Using LCT velocities, we approximate $v^{\text {ac }}$ by averaging the tangential velocity component $v_{t}$ over the annulus shaded in green. The annulus is defined by its radius $R$ and half-width $s$. We choose $R=\Delta$ for $n=6$ and $s=2 \mathrm{Mm}$. 
selected that are centered at solar latitudes from $-60^{\circ}$ to $60^{\circ}$ in steps of $20^{\circ}$. They were tracked for $24 \mathrm{~h}$ each at a rate consistent with the solar rotation rate from Snodgrass (1984) at the center of the patch. The data cubes cross the central meridian at approximately half the tracking time. They were remapped using Postel's projection with a spatial sampling of $0.5 \operatorname{arcsec} \mathrm{px}^{-1}\left(0.348 \mathrm{Mm} \mathrm{px}^{-1}\right)$. The temporal cadence is $45 \mathrm{~s}$. We divided each data cube into three $8 \mathrm{~h}$ datasets. The $x$ direction of the remapped images points to the west, the $y$ direction points to the north.

For further comparison, we also used Dopplergrams from the Michelson Doppler Imager (MDI) on board the Solar and Heliospheric Observatory (SOHO) spacecraft (Scherrer et al. 1995). We chose 59 days of images taken in the MDI full-disk mode that overlap in time with the HMI data (8 May through 11 July 2010). We tracked and remapped the MDI Dopplergrams in the same manner as for HMI, although with a coarser spatial sampling of $2.0 \operatorname{arcsec} \mathrm{px}^{-1}\left(1.4 \mathrm{Mm} \mathrm{px}^{-1}\right)$.

\subsubsection{Flow velocity maps from local correlation tracking}

For the LCT, we used our own code with the HMI photospheric intensity images as input. Our code is similar to the Fourier local correlation tracking (FLCT) code by Fisher \& Welsch (2008), but uses another procedure to measure the peak positions of the crosscorrelation (described later in this section).

We removed the temporal mean image for every dataset and chose an output grid with a sampling of $2.5 \operatorname{arcsec} \mathrm{px}^{-1}\left(1.7 \mathrm{Mm} \mathrm{px}^{-1}\right)$, thus five times coarser than the input images. The size of the image subsets used for the cross-correlation is adapted to the width of the Gaussian the subsets are multiplied by. We chose $\sigma=2 \mathrm{Mm}$ for the Gaussian and a diameter of $4 \sigma=8 \mathrm{Mm}$ for the subsets in both the $x$ and $y$ directions. The subsets are separated in time by $\Delta t=45 \mathrm{~s}$ (the cadence), which is sufficiently small compared to the granules' evolution timescale. We averaged the cross-correlations over the whole $8 \mathrm{~h}$ dataset.

To measure the peak position $(\Delta x, \Delta y)$ of the cross-correlation, we calculated (separately for $x$ and $y$ directions) the parameters of a parabola matching the cross-correlation at the maximum and the adjacent pixels. To improve the estimate of the peak position, we translated the cross-correlation by $(-\Delta x,-\Delta y)$ using Fourier interpolation and iterated the parabolic fit. We repeated this procedure four times in total. The measured shifts converge quickly, the maximum additional shift in a fifth iteration is of the order $10^{-5} \mathrm{px}$ at $60^{\circ}$ latitude (corresponding to $0.2 \mathrm{~m} \mathrm{~s}^{-1}$ or less), and the root mean square of the additional velocity shift is less than $0.02 \mathrm{~m} \mathrm{~s}^{-1}$. The measured peak position is the sum of the shifts measured in each step.

In Fig. 3.2 we compare the line-of-sight component $v_{\text {LOS }}$ of the LCT velocity with the velocity of an average Dopplergram, obtained by averaging Dopplergrams over the same time period as the LCT maps $(8 \mathrm{~h})$. Both images are for the same region at $40^{\circ}$ latitude around the central meridian. At this latitude, the average Dopplergrams are dominated by the horizontal flows that can be measured with LCT, but systematic effects like foreshortening are weak (see Appendix 3.10.1). We convolved the average Dopplergram with a Gaussian of width $\sigma / \sqrt{2} \approx 1.4 \mathrm{Mm}$ (FWHM roughly $3 \mathrm{Mm}$ ). This resembles the convolution of the intensity maps prior to computing the correlation of image subsets in the LCT. The chosen width maximizes the correlation coefficient between the average 
3 Paper II: Spatially resolved vertical vorticity in solar supergranulation using helioseismology and local correlation tracking

Dopplergram and the LCT image. In addition, we interpolated the average Dopplergram onto the coarser LCT grid and at each pixel subtracted the mean velocity over the map. To remove the residual rotation signal, we further subtracted a linear gradient in the $x$ direction that we obtained from a least-squares fit of $v_{x}$ averaged over $y$. The LCT line-of-sight velocity component was computed from $v_{x}$ and $v_{y}$. The $v_{y}$ map showed a linear gradient in the $y$ direction leading to an average velocity difference of about $-200 \mathrm{~m} \mathrm{~s}^{-1}$ between the bottom and the top of the map. This gradient is presumably due to the "shrinking Sun" effect, which has been discussed in Lisle \& Toomre (2004), although for LCT of Dopplergrams (a short description is also given in Appendix 3.10.1). The gradient (and the mean over the $v_{y}$ map) was removed before computing $v_{\text {LOS }}$.

The processed $v_{\text {LOS }}$ maps from direct Doppler data and LCT agree well (correlation coefficient 0.94 ). The scatter plot shows that on average the velocity values from LCT are slightly larger (by a factor 1.08) than from the Dopplergrams. This is different from what other authors have reported. De Rosa \& Toomre (1998) measured a slope of 0.89 and later (De Rosa \& Toomre 2004) 0.69 using their LCT code and SOHO/MDI Dopplergrams. Rieutord et al. (2001) and Verma et al. (2013) found that LCT underestimates the real velocities in convection simulations (however at smaller spatial scales than we are studying).

\subsubsection{Travel-time maps for horizontal divergence and vertical vortic- ity}

As input for the travel-time measurements, we used the HMI Dopplergrams. In Fourier space, we filtered the $8 \mathrm{~h}$ datasets to select either the f-mode or $\mathrm{p}_{1}$-mode ridge. The filters consist of a raised cosine function with a plateau region in frequency around the ridge maximum for every wavenumber $k$. Additionally, power for $k R_{\odot}<300$ and $k R_{\odot}>2600$ (f modes) and for $k R_{\odot}<180$ and $k R_{\odot}>1800$ ( $p_{1}$ modes) respectively is discarded. The symbol $R_{\odot}$ denotes the solar radius. The filter details are given in Appendix 3.8 .

We computed the cross-correlation $C$ from each filtered Doppler dataset in temporal Fourier space using

$$
C\left(\mathbf{r}_{1}, \mathbf{r}_{2}, \omega\right)=h_{\omega} \phi_{T}^{*}\left(\mathbf{r}_{1}, \omega\right) \phi_{T}\left(\mathbf{r}_{2}, \omega\right)
$$

where $\omega$ denotes the angular frequency, $h_{\omega}=2 \pi / T$ (with $T=8 \mathrm{~h}$ ) is the frequency resolution, and $\phi_{T}$ is the temporal Fourier transform of the filtered dataset (multiplied by the temporal window function).

For each dataset, we measured travel times $\tau^{\text {oi }}$ with an annulus radius of $10 \mathrm{Mm}$ and $\tau^{\text {ac }}$ with the parameters $\Delta=10 \mathrm{Mm}$ and $n=6$ (regular hexagon). We rotated the hexagon structure successively three times by an angle of $15^{\circ}$ to obtain four $\tau^{\text {ac }}$ measurements for the same dataset that are only weakly correlated (see Langfellner et al.|2014, for details). Averaging over these measurements yields a higher signal-to-noise ratio $(\mathrm{S} / \mathrm{N})$. We used the linearized travel-time definition by Gizon \& Birch (2004) and a sliding reference cross-covariance that we obtained by averaging $C$ over the entire map. 

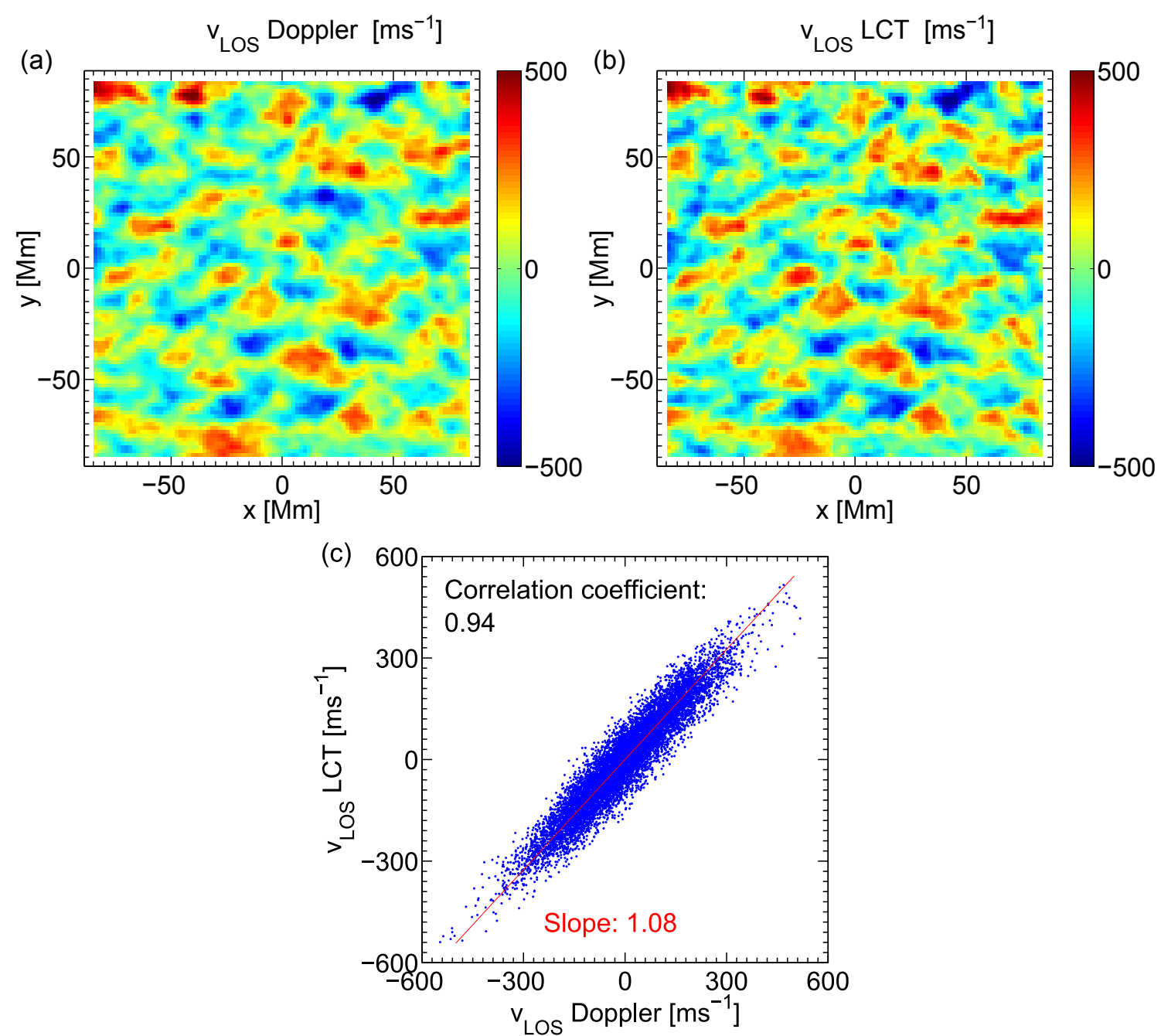

Figure 3.2: Comparison of line-of-sight velocity from two different data products at $40^{\circ}$ solar latitude on 6 June 2010. a) HMI Dopplergram averaged over $8 \mathrm{~h}$. The map was convolved with a Gaussian of $\sigma / \sqrt{2} \approx 1.4 \mathrm{Mm}$ and subsampled to match the coarser LCT resolution. The mean over the map and a linear function in the $x$ direction (parameters determined by a least-squares fit) were subtracted. b) LCT map from HMI intensity images, averaged over $8 \mathrm{~h}$. The line-of-sight velocity component was computed from the $v_{x}$ and $v_{y}$ components. For $v_{y}$, the mean over the map and a linear function in the $y$ direction were subtracted. c) Scatter plot of the two maps. The Pearson correlation coefficient is 0.94 . The red line shows the direction of largest scatter and crosses the origin. It is a best-fit line in the sense that it minimizes the sum of squared distances of the points perpendicular to the line (Pearson 1901). This is different from linear regression, where no error in the $x$ coordinate is assumed and only the sum of squared distances in the $y$ coordinate is minimized. The slope of the red line is 1.08 ; the error in the direction of lowest scatter is $33.9 \mathrm{~m} \mathrm{~s}^{-1}$. 


\subsection{Comparison of horizontal divergence and vertical vor- ticity from TD and LCT}

We now want to compare the measurements of horizontal divergence and vertical vorticity from TD and LCT for HMI. For TD, we use $\tau^{\mathrm{oi}}$ and $\tau^{\mathrm{ac}}$ as divergence- and vorticitysensitive quantities. For LCT, we can directly compute $\operatorname{div}_{h}=\partial_{x} v_{x}+\partial_{y} v_{y}$ and $\omega_{z}=$ $\partial_{x} v_{y}-\partial_{y} v_{x}$ from the $v_{x}$ and $v_{y}$ maps. To compute the derivatives of the LCT velocities, we apply Savitzky-Golay filters (Savitzky \& Golay 1964) for a polynomial of degree three and a window length of 15 pixels (about $5 \mathrm{Mm}$, with a FWHM of about $3 \mathrm{Mm}$ of the smoothing kernel). The Savitzky-Golay filters smooth out variations in the derivatives on spatial scales below the LCT resolution.

In the case of vorticity, we can also attempt a more direct comparison of TD and LCT. Consider the horizontal velocity field in 2D polar coordinates around $\mathbf{r}$ (see Fig. 3.1 c). Instead of using $v_{x}$ and $v_{y}$ for LCT, we can study the velocity component in the radial (divergent) direction, $v_{r}$, and the component in the tangential (anticlockwise) direction, $v_{t}$. The travel time $\tau^{\text {ac }}$ essentially measures $v_{t}$ averaged over the closed contour. The traveltime $\tau^{\text {ac }}$ is built up of point-to-point components $\tau^{\text {diff }}$ that capture the flow component that is parallel to the line connecting the two measurement points. The velocity magnitude that corresponds to the travel time $\tau^{\text {diff }}$ can roughly be estimated by calibration measurements using a uniform flow (Appendix 3.9). We use this calibration to convert $\tau^{\text {ac }}$ travel times into flow velocities and call the result $v^{\text {ac }}$. We note, however, that convective flows are highly turbulent, and thus a conversion factor obtained from uniform flows has to be treated with caution. Additionally, the conversion factor is sensitive to the details of the ridge filter (Appendix 3.10.3). We also note that because no inversion is applied, the velocities $v^{\text {ac }}$ represent an average over a depth range given by travel-time sensitivity kernels. For $\mathrm{f}$ modes, the range is from the surface to a depth of about $2 \mathrm{Mm}$, with a maximum of sensitivity near the surface, and for $\mathrm{p}_{1}$ modes from the surface to roughly $3 \mathrm{Mm}$, with one maximum near the surface and another one at a depth of about $2 \mathrm{Mm}$ (see, e.g., Birch \& Gizon 2007). With LCT, we approximate $v^{\text {ac }}$ by averaging $v_{t}$ over a hard-edge annulus with radius $R=10 \mathrm{Mm}$ and half-width $s=2 \mathrm{Mm}$ (see Fig. 3.1 d). The annulus width roughly corresponds to the width of travel-time sensitivity kernels (see, e.g., Jackiewicz et al. 2007).

For the divergence-sensitive measurements, this comparison is not possible without an inversion of the $\tau^{\mathrm{oi}}$ maps. Therefore, we limit our comparison to TD $\tau^{\mathrm{oi}}$ and LCT $\operatorname{div}_{h}$ in the following.

\subsubsection{Spatial power spectra of horizontal divergence and vertical vor- ticity}

From the TD $\tau^{\mathrm{oi}}$ and $\tau^{\mathrm{ac}}$ maps as well as the LCT $\operatorname{div}_{h}$ and $\omega_{z}$ maps, we calculated the spatial power spectra and averaged them over azimuth. The result for HMI is shown in Fig. 3.3. We rescaled the amplitude of the LCT power in order to show it together with the travel-time power.

For the divergence, the TD and LCT powers show a similar behavior at larger scales (except for $k R_{\odot}=25$, which corresponds to the map size). However, all three curves peak 
at different scales $-\mathrm{f}$ modes at $k R_{\odot}=120, \mathrm{p}_{1}$ modes at $k R_{\odot}=100$ and LCT at $k R_{\odot}=150$. The comparison with the curves for the TD noise model (Gizon \& Birch 2004) shows that the highest $\mathrm{S} / \mathrm{N}$ for the TD $\tau^{\mathrm{oi}}$ occurs at supergranulation scale, with $\mathrm{p}_{1}$ modes probing slightly larger scales than $\mathrm{f}$ modes. For LCT, no noise model is available that we know of. Thus it remains unclear if the peak of the power coincides with the peak of the S/N. For small scales $\left(k R_{\odot}\right.$ larger than 300$)$ the LCT power vanishes quickly, whereas the TD power reaches a noise plateau (f at $k R_{\odot}=500, \mathrm{p}_{1}$ at $k R_{\odot}=300$ ).

In the case of vorticity, the curves for TD and LCT look similar at large scales, although the power for LCT $\omega_{z}$ drops more quickly toward larger scales than for TD $\tau^{\mathrm{ac}}$. Compared to the divergence case, the peak positions are slightly shifted toward larger scales. However, the comparison with the TD noise model reveals that the $\mathrm{S} / \mathrm{N}$ does not have a peak at supergranulation scale but continues to increase toward larger scales (cf. Langfellner et al. 2014). At mid scales, the LCT power drops off only slowly, whereas the TD power quickly reaches the noise level (f at $k R_{\odot}=250, \mathrm{p}_{1}$ at $k R_{\odot}=200$ ). It is not clear if the considerably larger power of LCT $\omega_{z}$ at mid scales $\left(150<k R_{\odot}<500\right)$ is due to real flows or noise. At smaller scales, both TD curves behave more erratically. This happens, however, in a regime of almost pure noise. LCT power drops off quickly beyond $k R_{\odot}=400$.

\subsubsection{Maps of horizontal divergence and vertical vorticity}

For comparing maps of horizontal divergence and vertical vorticity, one point to consider is the different spatial sampling for TD and LCT maps. To correct for this, we interpolate the velocity maps derived from LCT onto the finer travel-time grid. In order to compare the maps on different spatial scales, we apply different band-pass filters to the individual maps in Fourier space. The individual filters are centered around $k R_{\odot}$ values of 50 through 400 in steps of 50. Each filter is one in a plateau region of width 50, centered around these values. Adjacent to both sides of the plateau are raised cosine flanks that make the filter smoothly reach zero within a $k R_{\odot}$ range of 50. Additionally, we employ a high-pass filter for $k R_{\odot}>400$. From all maps, we subtract the respective mean map over all 336 datasets prior to filtering.

Example $8 \mathrm{~h}$ maps for $\tau^{\mathrm{oi}}$ and $v^{\mathrm{ac}}$ from $\mathrm{f}$-mode travel times as well as $\operatorname{div}_{h}$ and $\omega_{z}$ from LCT are depicted in Fig. 3.4. The maps are filtered around $k R_{\odot}=100$. Note that for the sake of an easier comparison, we plotted $-\tau^{\mathrm{oi}}$ rather than $\tau^{\mathrm{oi}}$. For the flow divergence, all three maps are highly correlated. The average correlation coefficients over all 336 maps are 0.96 between LCT $\operatorname{div}_{h}$ and $-\tau^{\mathrm{oi}}$ for $\mathrm{f}$ modes and 0.92 between LCT $\operatorname{div}_{h}$ and $-\tau^{\mathrm{oi}}$ for $\mathrm{p}_{1}$ modes.

In the case of flow vorticity, the agreement of the LCT and TD maps is weaker than for the divergence. The average correlation coefficient over all 336 maps is 0.68 between LCT $\omega_{z}$ and f-mode $v^{\text {ac }}$ and 0.51 between LCT $\omega_{z}$ and $\mathrm{p}_{1}$-mode $v^{\text {ac }}$ (not shown). When comparing LCT $v^{\text {ac }}$ instead of $\omega_{z}$ with TD $v^{\text {ac }}$, the correlation coefficients are noticably higher $\left(0.75\right.$ for $f$ modes and 0.57 for $\mathrm{p}_{1}$ modes). The flow magnitudes are roughly comparable.

Table 3.1 shows the correlation coefficients between LCT and TD averaged over all datasets for all filters and including $\mathrm{p}_{1}$ modes. The error in the correlation coefficients is less than 0.01 . Note that the edges $(12 \mathrm{Mm})$ were removed from the maps before the cor- 

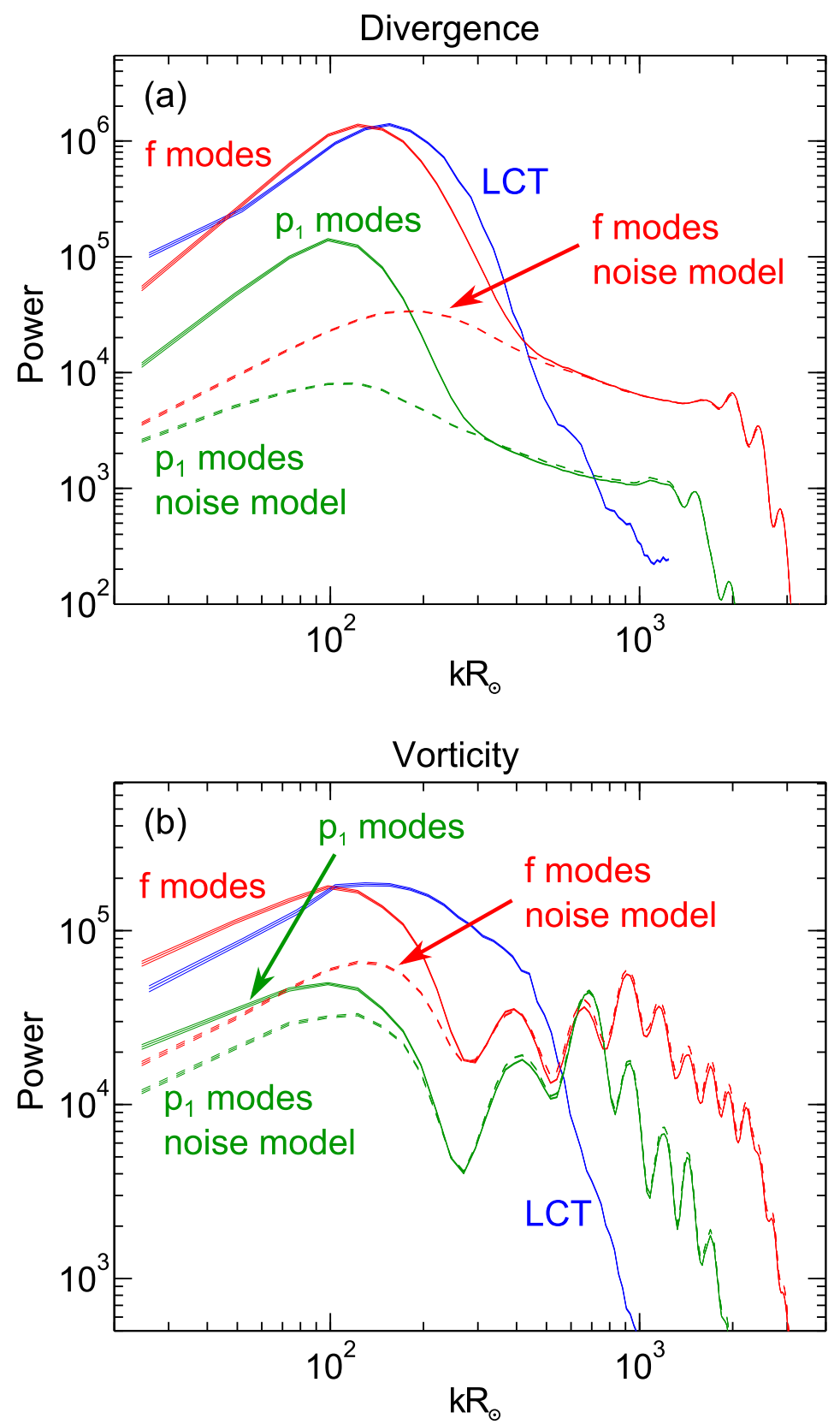

Figure 3.3: Power spectra (averaged over azimuth of wavevector $\mathbf{k}$ and 336 datasets) of TD travel-time maps, and LCT $\operatorname{div}_{h}$ and $\omega_{z}$ maps computed from HMI Dopplergrams and intensity images at the solar equator near disk center (distributed symmetrically between $7^{\circ}$ east and west of the central meridian). a) Divergence-sensitive travel times $\tau^{\mathrm{oi}}$ for $\mathrm{f}$ modes and $\mathrm{p}_{1}$ modes as well as LCT $\operatorname{div}_{h}$. b) Vorticity-sensitive travel times $\tau^{\text {ac }}$ for $\mathrm{f}$ modes and $\mathrm{p}_{1}$ modes as well as LCT $\omega_{z}$. The amplitudes of LCT $\operatorname{div}_{h}$ and $\omega_{z}$ power were rescaled to match the range of the travel-time power. The dashed lines represent noise models for the $f$ and $p_{1}$ modes based on Gizon \& Birch (2004). The thickness of the lines denotes the $1 \sigma$ error. 

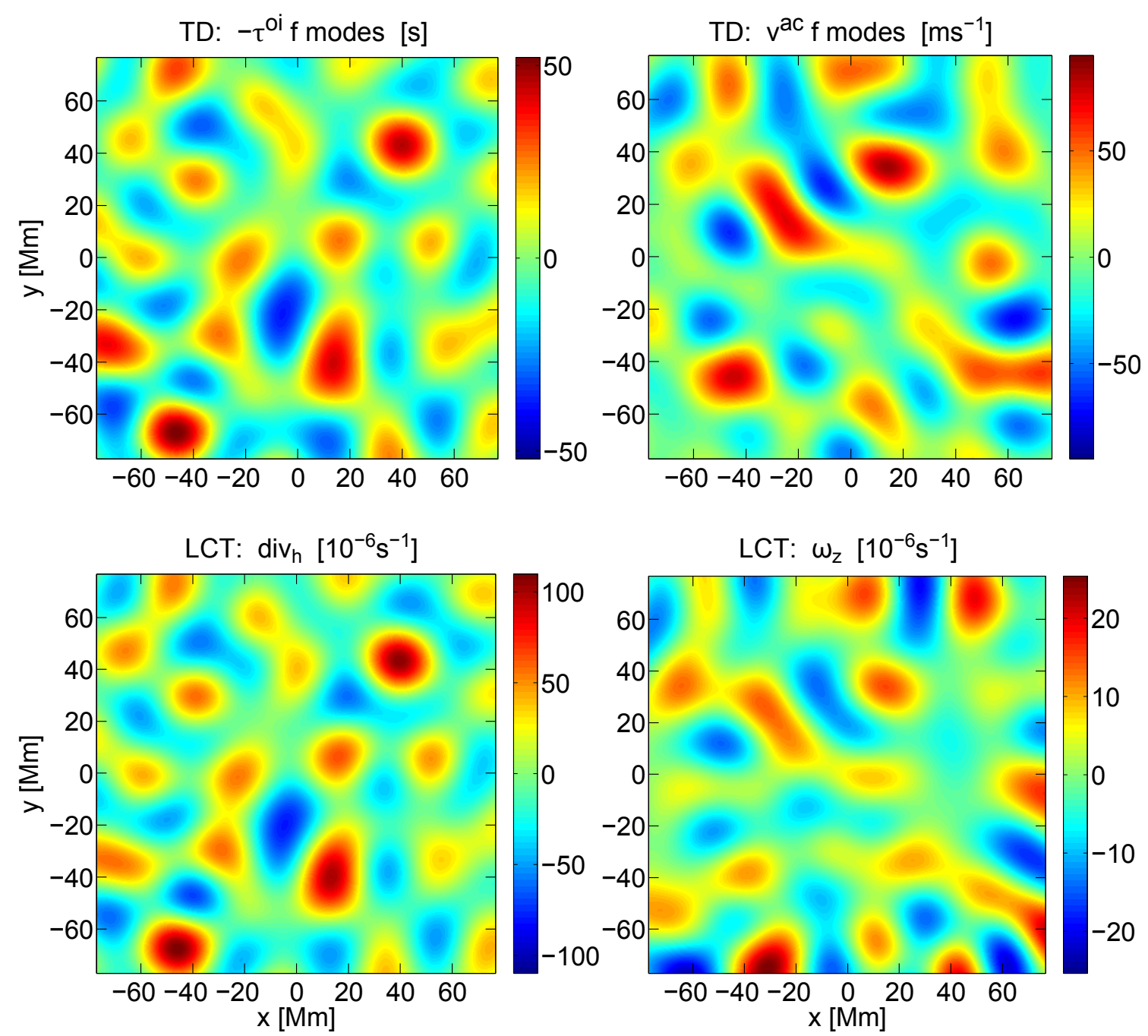

Figure 3.4: Comparison of TD and LCT maps at the equator. All maps are based on $8 \mathrm{~h}$ of HMI images (intensity and Doppler velocity) taken on 1 May 2010 and have been band-pass filtered around $k R_{\odot}=100$. The color bar limits are set to the maximum absolute value of the corresponding map and symmetrized around zero. Left column: Divergencesensitive travel times $\tau^{\mathrm{oi}}$ for f modes as well as LCT horizontal divergence $\operatorname{div}_{h}$. Right column: Circulation velocities $v^{\text {ac }}$ for TD as well as LCT vertical vorticity $\omega_{z}$. The TD $\tau^{\text {ac }}$ maps were converted into velocity maps by pointwise multiplication with a constant factor $-5.62 \mathrm{~m} \mathrm{~s}^{-2}$ (f modes) and $-11.1 \mathrm{~m} \mathrm{~s}^{-2}$ ( $\mathrm{p}_{1}$ modes), see Appendix 3.9 for details. 
3 Paper II: Spatially resolved vertical vorticity in solar supergranulation using helioseismology and local correlation tracking

Table 3.1: Correlation between LCT maps and TD travel-time maps derived from HMI intensity images and Dopplergrams.

\begin{tabular}{cc|ccc}
\hline \hline \multirow{2}{*}{$\begin{array}{c}\text { Modes } \\
\text { (TD) }\end{array}$} & $k R_{\odot}$ & \multicolumn{3}{|c}{ Correlation coeff. between LCT and TD } \\
LCT div $d_{h}$ & LCT $\omega_{z}$ & LCT $v^{\text {ac }}$ \\
TD $-\tau^{\text {oi }}$ & TD $-\tau^{\text {ac }}$ & TD $-\tau^{\text {ac }}$ \\
\hline $\mathrm{f}$ & 50 & 0.93 & 0.70 & 0.77 \\
& 100 & 0.96 & 0.68 & 0.75 \\
& 150 & 0.96 & 0.63 & 0.68 \\
& 200 & 0.94 & 0.53 & 0.57 \\
& 250 & 0.89 & 0.31 & 0.30 \\
& 300 & 0.78 & -0.01 & 0.14 \\
& 350 & 0.58 & -0.09 & 0.23 \\
& 400 & 0.31 & -0.06 & 0.23 \\
& $>400$ & 0.02 & 0.00 & 0.03 \\
\hline $\mathrm{p}_{1}$ & 50 & 0.90 & 0.53 & 0.59 \\
& 100 & 0.92 & 0.51 & 0.57 \\
& 150 & 0.89 & 0.44 & 0.50 \\
& 200 & 0.83 & 0.33 & 0.38 \\
& 250 & 0.66 & 0.13 & 0.19 \\
& 300 & 0.36 & -0.06 & 0.11 \\
& 350 & 0.15 & -0.05 & 0.11 \\
& 400 & 0.04 & -0.02 & 0.08 \\
& $>400$ & 0.00 & 0.00 & 0.01 \\
\hline
\end{tabular}

relation coefficients were computed. For the flow divergence, the correlation coefficients are almost constantly high for smaller $k R_{\odot}$ values. In the range $k R_{\odot}=300-400$, the correlation coefficient between LCT $\operatorname{div}_{h}$ and $-\tau^{\text {oi }}$ for f modes rapidly decreases from 0.78 to 0.31 . For LCT and $\mathrm{p}_{1}$ modes, the correlation coefficient decreases from 0.83 to 0.15 from $k R_{\odot}=200-350$. For the high-pass filters, the LCT and TD maps are completely uncorrelated.

In the case of vorticity, the correlation decreases rapidly for both $\mathrm{f}$ and $\mathrm{p}_{1}$ modes at $k R_{\odot}=200$. Again, the LCT and TD maps are uncorrelated for large $k R_{\odot}$. The correlation coefficients for LCT $v^{\text {ac }}$ are significantly higher than for LCT $\omega_{z}$.

The dependence of the correlation coefficients on spatial scale conceptually agrees well with the power spectra in Fig. 3.3. There is a high correlation on large scales where the observed TD travel-time power clearly exceeds the power of the TD noise model. On the other hand, the very low correlation on smaller scales reflects that the power of TD observations and noise model are almost equal.

Qualitatively, the correlation coefficients are comparable with the value 0.89 from De Rosa et al. (2000) who obtained travel-time and LCT velocity maps from SOHO/MDI Dopplergrams and smoothed the divergence maps by convolving with a Gaussian with FWHM 6.2 Mm. 


\subsection{Net vortical flows in the average supergranule}

The major goal of this paper is to spatially resolve the vorticity of the average supergranule at different solar latitudes. In the following, we describe the averaging process and show average divergence and vorticity maps.

\subsubsection{Obtaining maps of the average supergranule}

To construct the average supergranule, we started by identifying the location of supergranule outflows and inflows in f-mode $\tau^{\mathrm{oi}}$ maps from HMI and MDI. We smoothed the maps by removing power for $k R_{\odot}>300$ and applied an image segmentation algorithm (Hirzberger et al. 2008). The coordinates of the individual supergranules were used to align maps of various data products. For each identified position, we translated a copy of the map to move the corresponding supergranule to the map center. These translated maps were then averaged. At each latitude, we averaged over roughly 3000 supergranules in total for HMI (1500 supergranules for MDI). Supergranules closer than $8 \mathrm{Mm}$ to the map edges were discarded.

We produced maps for the average supergranule outflow and inflow from $\tau^{\mathrm{oi}}$ and $\tau^{\mathrm{ac}}$ travel-time maps as well as LCT $v_{x}$ and $v_{y}$ maps. Prior to the averaging process, the LCT maps were spatially interpolated onto the (finer) travel-time grid. For all maps, we subtracted the mean map over all 336 HMI datasets (177 datasets in the case of MDI). This removes signal that does not change with time (or changes only slowly), including differential rotation. Additionally, we removed power for $k R_{\odot}>300$ by applying a lowpass filter in Fourier space.

The resulting average $\tau^{\mathrm{ac}}$ maps were converted into $v^{\mathrm{ac}}$ maps. From the LCT $v_{x}$ and $v_{y}$ maps for the average supergranule, we computed $\operatorname{div}_{h}, \omega_{z}$, and $v^{\text {ac }}$. The Savitzky-Golay filters that we employed to compute the spatial derivatives smooth out step artefacts from the image alignment process, yet preserve the signal down to the resolution limit of the LCT.

We corrected the $v^{\text {ac }}$ and $\omega_{z}$ maps for geometrical center-to-limb systematics (unless stated otherwise). We measured these effects using HMI and MDI observations west and east of the disk center, at relative longitudes corresponding to the latitudes of the regular observations. The idea is that any difference (beyond the noise background) between maps at disk center and a location west or east from disk center is due to geometrical center-to-limb systematics. These systematics only depend on the distance to the disk center. Therefore, our raw measurements of $v^{\text {ac }}$ and $\omega_{z}$ that we obtained north and south of the equator should be affected by the systematics in the same way as measurements west and east of the disk center. We corrected the raw data by subtracting the $v^{\text {ac }}$ and $\omega_{z}$ maps west and east of the disk center. This approach is analogous to Zhao et al. (2013) who used the method to correct measurements of the meridional circulation. Figure 3.5 illustrates the correction process for $v^{\text {ac }}$ maps at $60^{\circ}$ latitude. The correction is particularly important for LCT at high latitudes. We note that the measured center-to-limb systematics at lower latitudes (up to $40^{\circ}$ north and south) are much weaker and only lead to a mild correction of the $v^{\text {ac }}$ and $\omega_{z}$ maps. A further discussion of the center-to-limb systematics can be found in Appendix 3.10 .1 


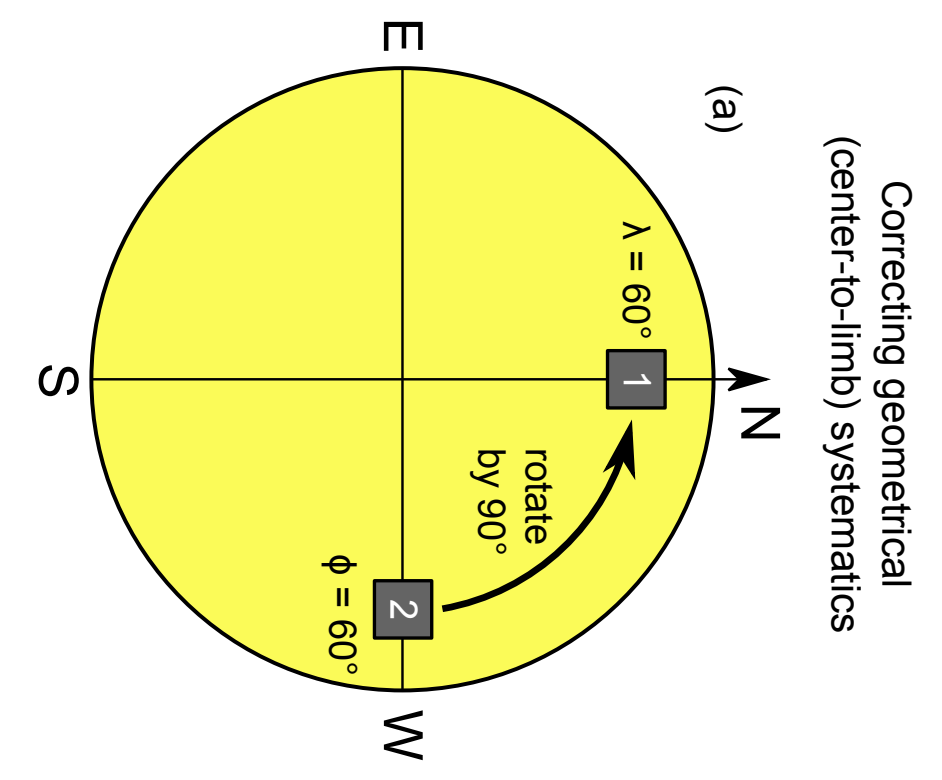

य

商

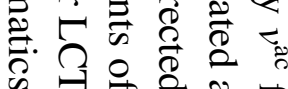

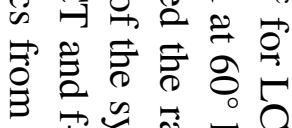

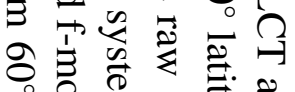
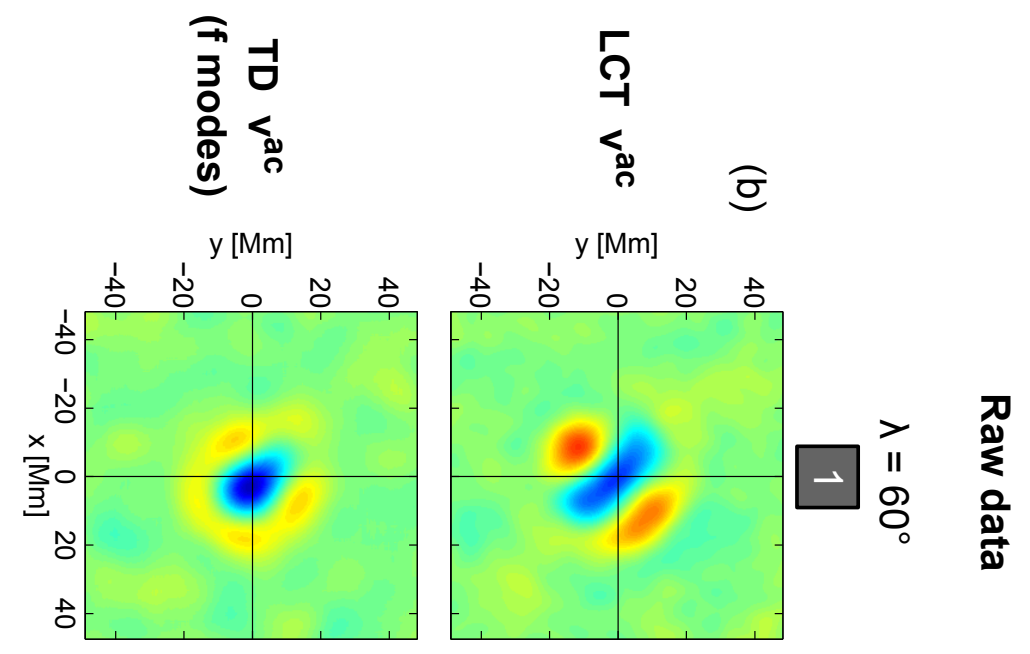

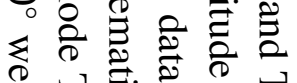

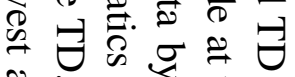

卷完帛

ஓ ซ

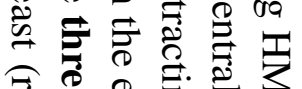

ชิ

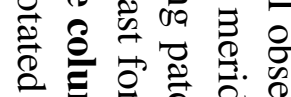

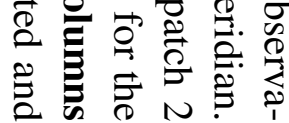
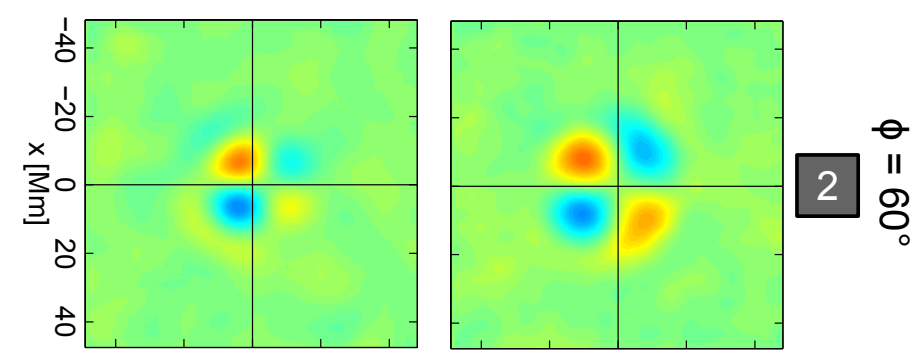

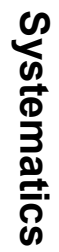
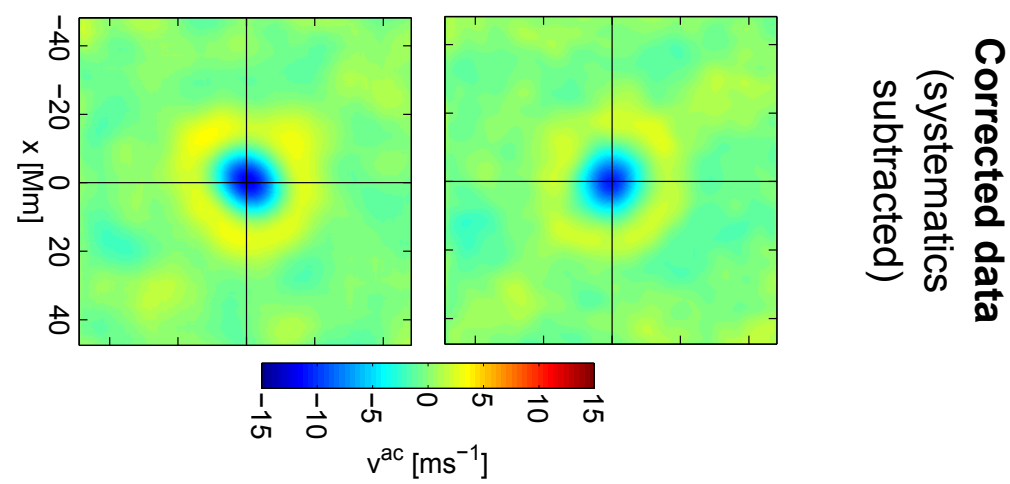


\subsubsection{Latitudinal dependence of the vertical vorticity in outflow re- gions}

Figure 3.6 shows the circulation velocity $v^{\text {ac }}$ in the average supergranule outflow region for LCT and f-mode TD for latitudes from $-60^{\circ}$ to $60^{\circ}$, in steps of $20^{\circ}$. For comparison, the left column shows the horizontal divergence $\operatorname{div}_{h}$ from LCT. At all latitudes, there is a peak of positive divergence at the origin. All divergence peaks are surrounded by rings of negative divergence. This suggests that on average every supergranule outflow region is isotropically surrounded by inflow regions. The strength of the divergence peak slightly decreases toward higher latitudes. Furthermore, the divergence peaks are slightly shifted toward the equator at high latitudes (by about $0.7 \mathrm{Mm}$ at $\pm 60^{\circ}$ ). These effects are presumably due to center-to-limb systematics.

The $v^{\text {ac }}$ maps (center and right columns) show negative peaks (clockwise motion) in the northern hemisphere and positive peaks (anticlockwise motion) in the southern hemisphere. The peaks are surrounded by rings of opposite sign, as for the divergence maps. There is a remarkable agreement between LCT and TD in both shape and strength of the peak structures. At the solar equator, no peak and ring structures are visible; however, we note that the LCT and TD $v^{\mathrm{ac}}$ maps at the equator are still correlated. This shows that the "noise" background is due to real flows rather than measurement noise that is dependent on the technique.

To study the latitudinal dependence of the observed and corrected signal in more detail, we plot in Fig. 3.7 a the peak velocity $v^{\text {ac }}$ from Fig. 3.6, including $\mathrm{p}_{1}$-mode TD, as a function of solar latitude (lines). The peak velocity shows an overall decrease from south to north, with a zero-crossing at the equator. The curves are antisymmetric with respect to the origin. The peak velocities have similar values at a given latitude, with f-mode velocities appearing slightly stronger than LCT and $\mathrm{p}_{1}$-mode velocities (in this order). The highest velocities are slightly above $10 \mathrm{~m} \mathrm{~s}^{-1}$. Figure $3.7 \mathrm{p}$ shows the peak magnitude in maps of the vertical vorticity $\omega_{z}$, as measured from LCT. The overall appearance is similar to the circulation velocities $v^{\text {ac }}$. The highest absolute vorticity value is about $5 \times 10^{-6} \mathrm{~s}^{-1}$.

Figure 3.8 a shows cuts through $y=0$ for the maps of LCT and TD $v^{\text {ac }}$ (including $\mathrm{p}_{1}$ modes) at $40^{\circ}$ latitude. We use this latitude because the $\mathrm{S} / \mathrm{N}$ in the $v^{\text {ac }}$ and $\omega_{z}$ peaks is high compared to other latitudes, whereas the measurements are only mildly affected by center-to-limb systematics. The velocity magnitudes and shapes of the curves are comparable for the three cases. For the LCT and f-mode curves, an asymmetry in the west-east direction is visible. This means that the ring structures surrounding the peaks in the $v^{\text {ac }}$ maps are stronger in the west than in the east. The FWHM is about $13 \mathrm{Mm}$ in all cases. The peaks are very slightly shifted eastwards. However, this east shift does not appear to be a general feature at all latitudes. Mostly, the shifts are consistent with random fluctuations. Partly, the shifts might also be due to other effects, for instance an incomplete removal of center-to-limb systematics.

For comparison, the FWHM of the $\tau^{\text {oi }}$ peak structure is about $13 \mathrm{Mm}$ for $\mathrm{p}_{1}$ modes, compared to about $11 \mathrm{Mm}$ for f-mode $\tau^{\text {oi }}$. The horizontal divergence $\operatorname{div}_{h}$ from LCT at $40^{\circ}$ latitude peaks at about $170 \times 10^{-6} \mathrm{~s}^{-1}$ with a FWHM of about $10 \mathrm{Mm}$.

From the $v^{\text {ac }}$ peak velocities, we can estimate the average vorticity $\left\langle\omega_{z}\right\rangle_{A}$ over the circular area $A$ of radius $R=10 \mathrm{Mm}$ that is enclosed by the $\tau^{\text {ac }}$ measurement contour (see 
3 Paper II: Spatially resolved vertical vorticity in solar supergranulation using helioseismology and local correlation tracking

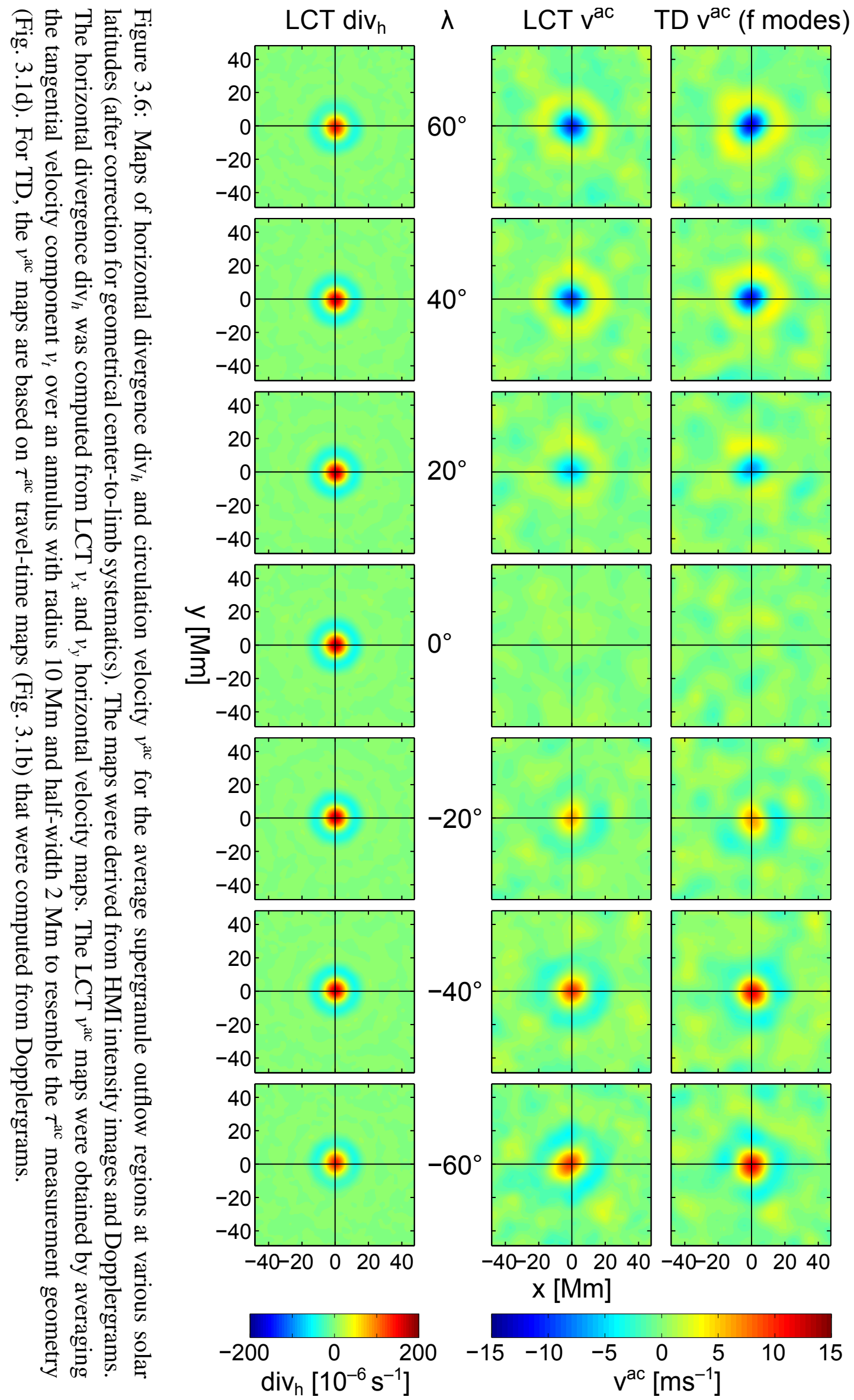



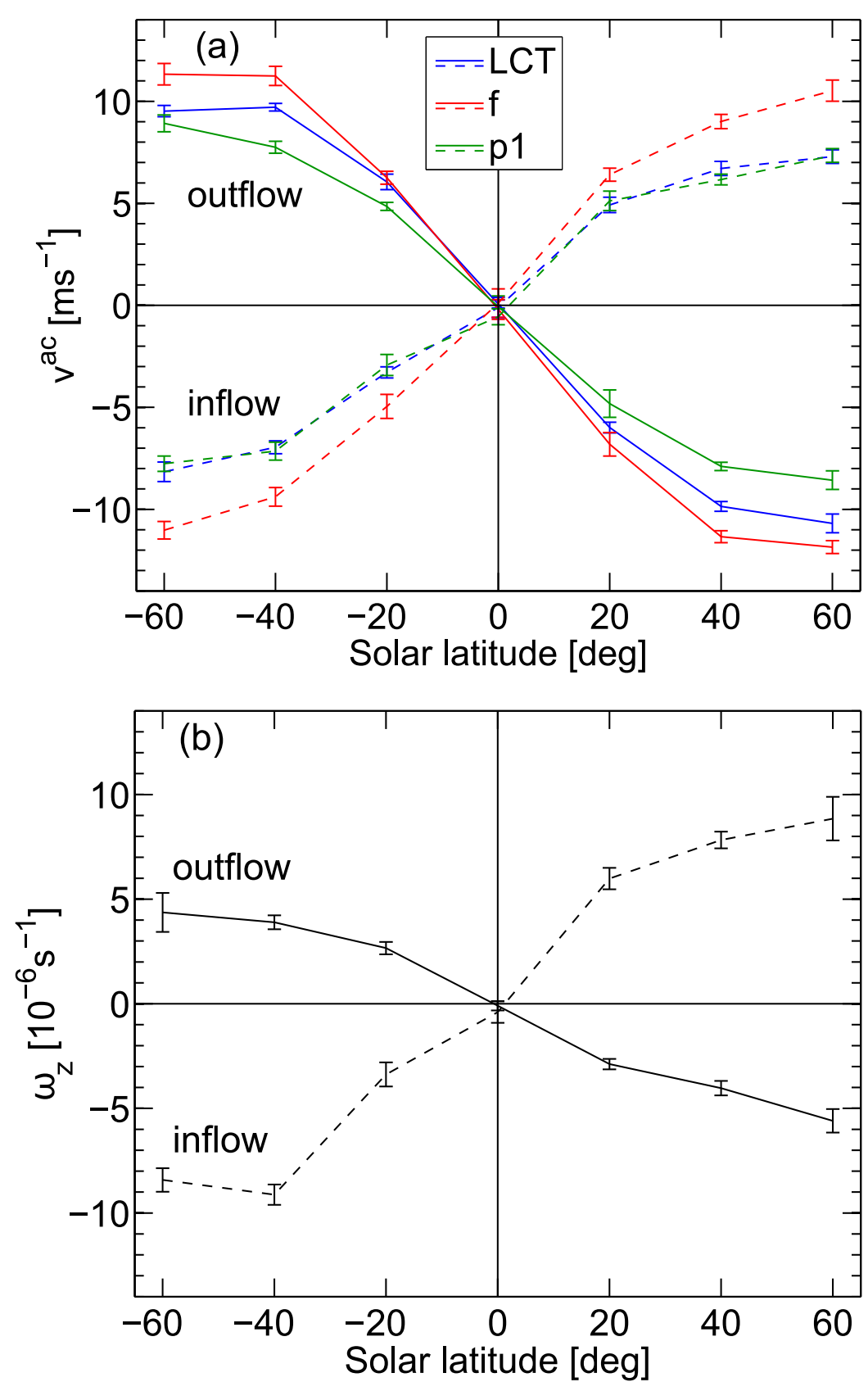

Figure 3.7: Peak $v^{\text {ac }}$ and $\omega_{z}$ values for the average supergranule at different solar latitudes. a) Circulation velocity $v^{\text {ac }}$ for LCT, f modes and $\mathrm{p}_{1}$ modes. b) Vertical component of flow vorticity $\omega_{z}$ obtained from LCT. Solid lines are for the average supergranule outflow region, dashed lines for the average supergranule inflow region. At $0^{\circ}$ latitude, the values at the map center are shown instead of the peak values. The error bars were computed by dividing the 336 datasets into eight parts and measuring the variance of $v^{\text {ac }}$ and $\omega_{z}$ at the peak positions over the eight parts. 
Fig. 3.1). The average vorticity is given by

$$
\left\langle\omega_{z}\right\rangle_{A}=\frac{\Gamma}{A} \approx \frac{2 v^{\mathrm{ac}}}{R},
$$

where $\Gamma$ is the flow circulation along the $\tau^{\text {ac }}$ measurement contour that we approximated with $\Gamma \approx 2 \pi R v^{\text {ac }}$. By taking the $v^{\text {ac }}$ peak values, we obtain $\left\langle\omega_{z}\right\rangle_{A} \approx-2.4 \times 10^{-6} \mathrm{~s}^{-1}$ for the f modes, $\left\langle\omega_{z}\right\rangle_{A} \approx-1.6 \times 10^{-6} \mathrm{~s}^{-1}$ for the $\mathrm{p}_{1}$ modes, and $\left\langle\omega_{z}\right\rangle_{A} \approx-2.0 \times 10^{-6} \mathrm{~s}^{-1}$ for LCT. Thus the average vorticity in the circular region is roughly half the peak vorticity at $40^{\circ}$ latitude.

\subsubsection{Inflow regions}

So far we have discussed vortical flows around supergranule outflow centers. It is interesting though to compare the magnitude and profile of these flows with the average inflow regions, which have a different geometrical structure (connected network instead of isolated cells). Analogously to Fig. 3.6 for the outflows, Fig. 3.9 shows maps of $\operatorname{div}_{h}$ and $v^{\mathrm{ac}}$ around the average supergranule inflow center. As for the outflows, the $v^{\mathrm{ac}}$ maps from TD and LCT agree very well at all analyzed latitudes. The peaks in the $v^{\text {ac }}$ maps have the opposite sign compared to the outflows. This indicates that flows are preferentially in the clockwise (anticlockwise) direction in the average supergranular outflow region and anticlockwise (clockwise) in the average inflow region in the northern (southern) hemisphere. Cuts through $y=0$ of the $v^{\text {ac }}$ maps at $40^{\circ}$ latitude are shown in Fig. $3.8 \mathrm{~b}$. The $v^{\text {ac }}$ curves have the same shape as the corresponding curves for the average outflow center (with a FWHM of 14 to $16 \mathrm{Mm}$ ) but the peak flow magnitude is reduced and the sign is switched. As in the case of the outflows, the ring structures are stronger on the west side than on the east side.

The horizontal flow divergence $\operatorname{div}_{h}$ in the average inflow is similar to the average outflow (about the same FWHM) but with reversed signs and reduced magnitude. The peak divergence is about $-120 \times 10^{-6} \mathrm{~s}^{-1}$ at $40^{\circ}$ latitude with a FWHM of about $10 \mathrm{Mm}$. As in the case of the outflows, there is a systematic decrease in peak magnitude and a slight equatorward shift of the $\operatorname{div}_{h}$ peak at high latitudes.

The latitude dependence of the $v^{\text {ac }}$ peak values for the average supergranule inflow region (dashed lines in Fig. 3.7a) is almost mirror-symmetric to the outflow regions. The values are slightly smaller compared to the average outflow, with a ratio inflow/outflow of $-0.87 \pm 0.03$ for the $f$ modes, $-0.85 \pm 0.06$ for the $\mathrm{p}_{1}$ modes, and $-0.72 \pm 0.05$ for the LCT $v^{\text {ac }}$. In the case of $\omega_{z}$ (Fig. 3.7b), on the other hand, the ratio between the average inflow and outflow center is $-1.8 \pm 0.2$.

From the peak values of $v^{\text {ac }}$, we can estimate the average vorticity $\left\langle\omega_{z}\right\rangle_{A}$ over the circular area $A$ of radius $R=10 \mathrm{Mm}$ in the same way as for the outflow regions. We obtain $\left\langle\omega_{z}\right\rangle_{A} \approx 1.8 \times 10^{-6} \mathrm{~s}^{-1}$ for the f modes, $\left\langle\omega_{z}\right\rangle_{A} \approx 1.2 \times 10^{-6} \mathrm{~s}^{-1}$ for the $\mathrm{p}_{1}$ modes, and $\left\langle\omega_{z}\right\rangle_{A} \approx 1.4 \times 10^{-6} \mathrm{~s}^{-1}$ for LCT. The peak vorticity at $40^{\circ}$ latitude is therefore larger by a factor of about five compared to the vorticity averaged over the circular area.

\subsubsection{Dependence of the vertical vorticity on horizontal divergence}

The detection of net tangential flows in the average supergranule raises the question of how much the magnitudes of $v^{\text {ac }}$ and $\omega_{z}$ depend on the selection of supergranules. As a 

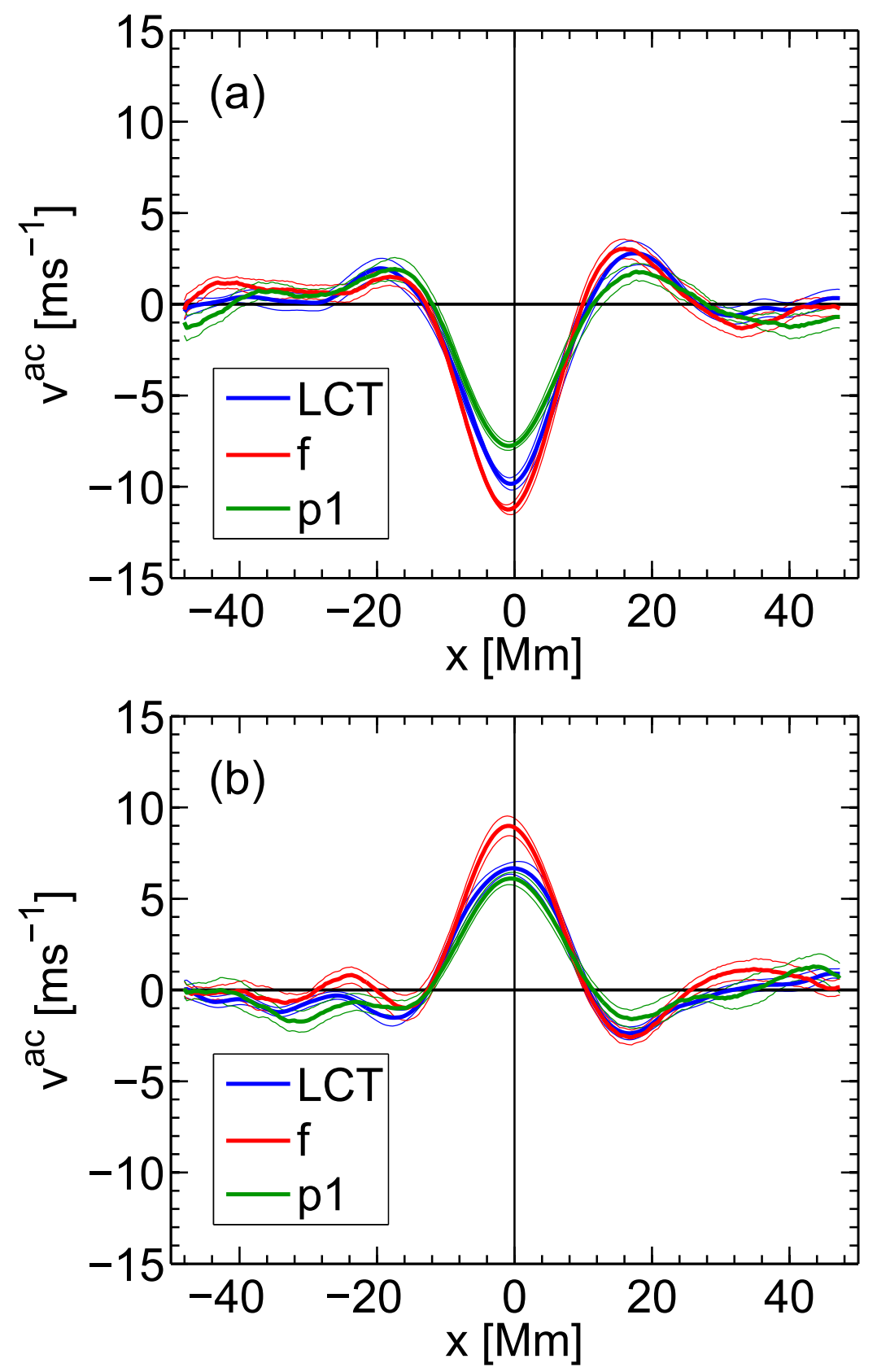

Figure 3.8: a) Cuts through the maps of the circulation velocity $v^{\text {ac }}$ for the average supergranule outflow region at $40^{\circ}$ latitude (shown in Fig. 3.6), at $y=0$. The TD and LCT maps were derived from HMI Dopplergrams and intensity images. The thin lines denote estimates of the variability of the data as obtained from dividing the 336 datasets into eight parts. The $1 \sigma$ level is shown. b) As a), but for the average supergranule inflow region at $40^{\circ}$ latitude.

test, we sort the identified supergranules at $40^{\circ}$ latitude from HMI with respect to their divergence strength, as measured by the peak f-mode $\tau^{\mathrm{oi}}$ of each supergranule. The sorted supergranules are assigned to four bins, which each contain roughly the same number of supergranules. The boundaries of the bins for f-mode $\tau^{\mathrm{oi}}$ are about $-96.9,-53.8,-42.1$, 
3 Paper II: Spatially resolved vertical vorticity in solar supergranulation using helioseismology and local correlation tracking

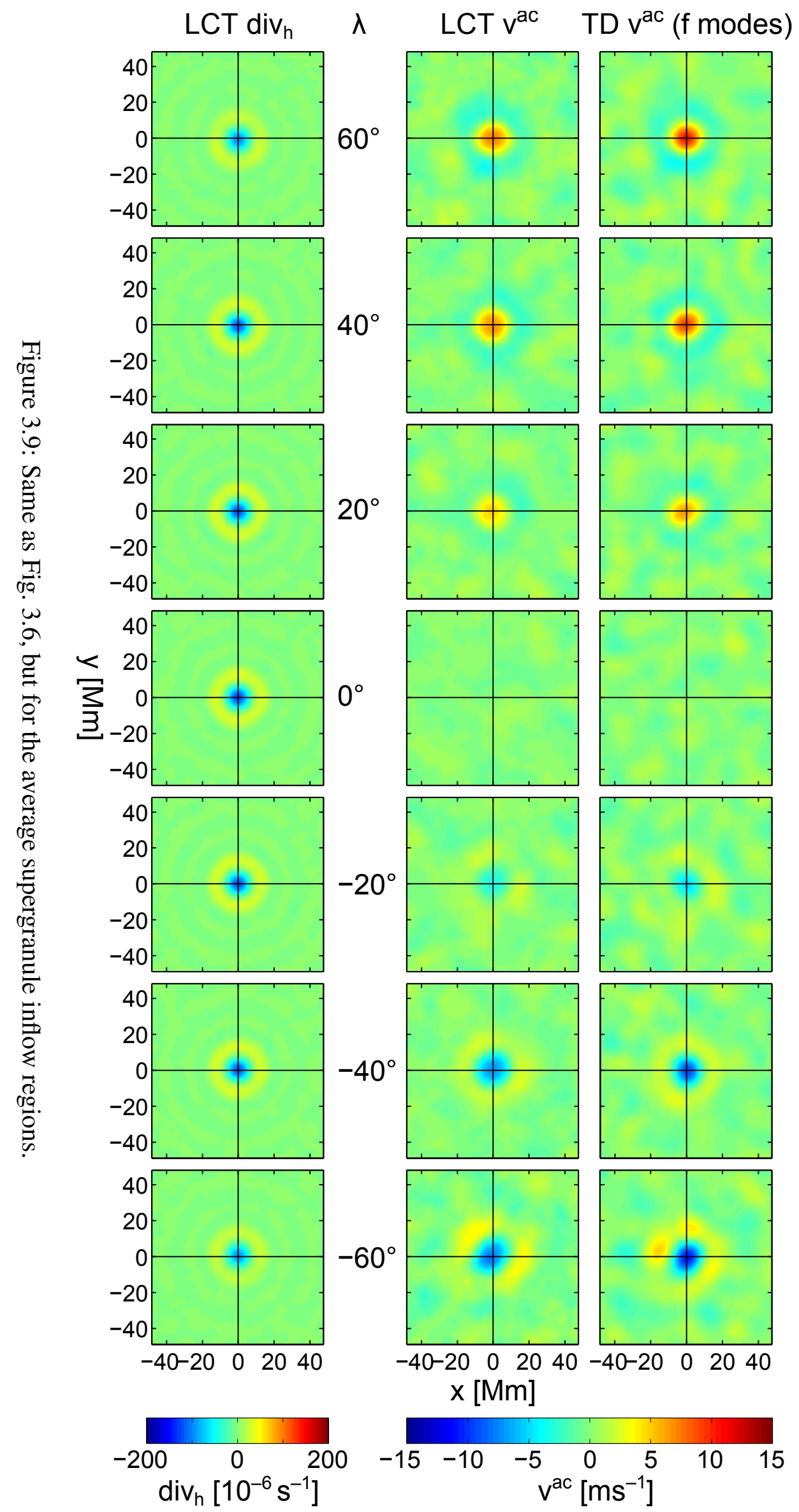



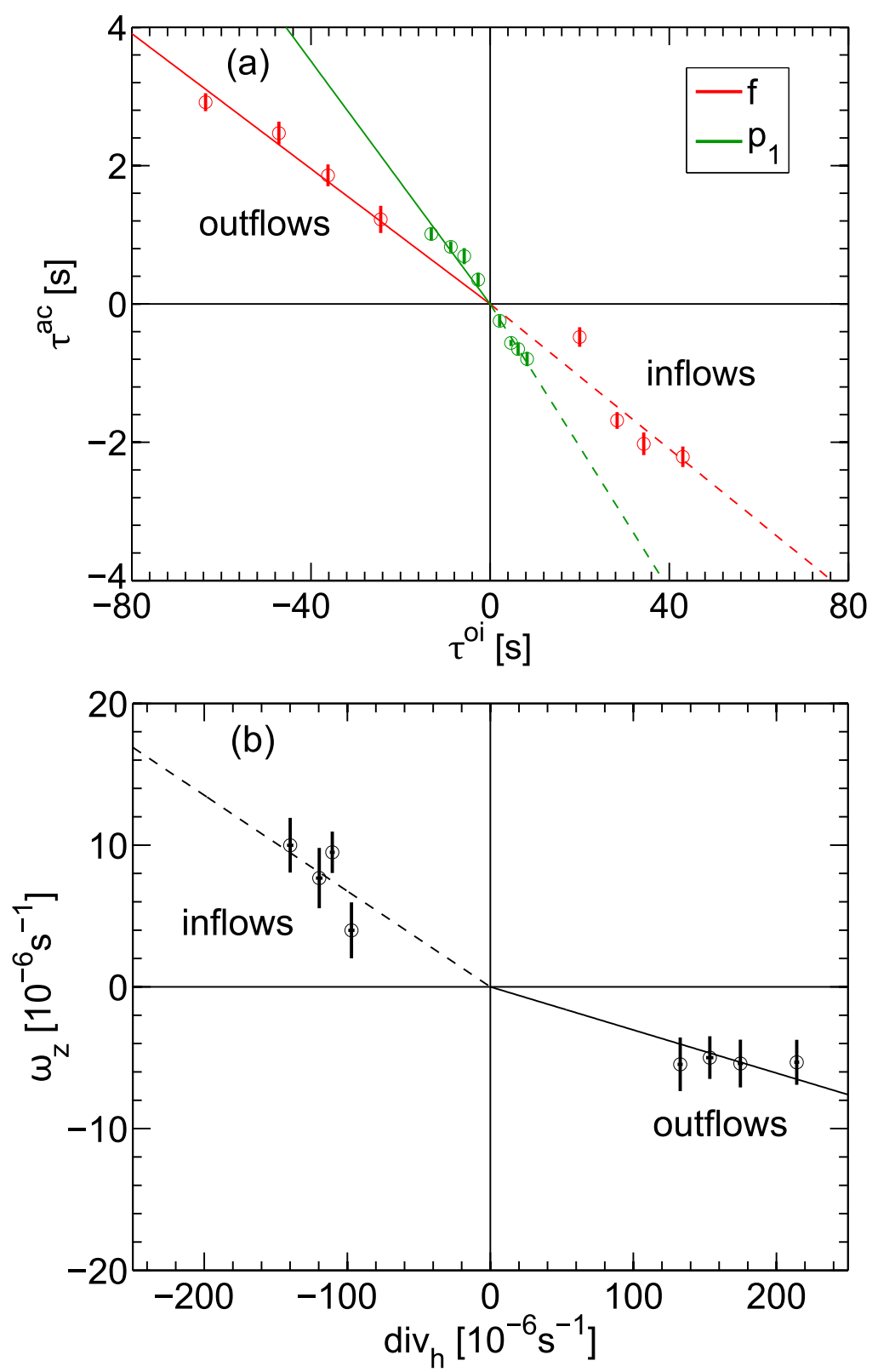

Figure 3.10: Vorticity as a function of divergence for the average supergranule at $40^{\circ}$ latitude from HMI data. a) Vorticity travel times $\tau^{\text {ac }}$ versus divergence travel times $\tau^{\text {oi }}$ for TD $\mathrm{f}$ modes and $\mathrm{p}_{1}$ modes. The upper left quadrant shows the values for the average outflows, the lower right quadrant for the average inflows. The solid and the dashed lines show least-squares fits of a linear function through the origin for outflows and inflows, respectively. The error bars were obtained by dividing the 336 datasets into eight parts. b) As a) but the peak $\omega_{z}$ versus the peak $\operatorname{div}_{h}$ from LCT is shown. We note that the quadrants depicting outflows and inflows are flipped compared to the travel times in a).

-31.5 , and $-16.0 \mathrm{~s}$ for the outflows and $67.5,38.3,32.1,26.0$, and $11.1 \mathrm{~s}$ for the inflows. We note that a simple scatter plot would be very noisy because the $v^{\text {ac }}$ and $\omega_{z}$ maps are dominated by turbulence.

For each bin, we computed the peak TD $\tau^{\mathrm{oi}}$ and $\tau^{\text {ac }}$ as well as LCT $\operatorname{div}_{h}$ and $\omega_{z}$ in the 
3 Paper II: Spatially resolved vertical vorticity in solar supergranulation using helioseismology and local correlation tracking

same way as for all identified supergranules that we discussed in the previous sections, but without the correction for center-to-limb systematics. In Fig. 3.10 a, we plot the peak $\tau^{\text {ac }}$ as a function of the peak $\tau^{\mathrm{oi}}$ from $\mathrm{f}$ modes and $\mathrm{p}_{1}$ modes both for outflows and inflows. The magnitude of $\tau^{\text {ac }}$ clearly increases with $\tau^{\mathrm{oi}}$. The ratio $\tau^{\mathrm{ac}} / \tau^{\mathrm{oi}}$ is roughly constant. Only the f-mode bin for the weakest inflows deviates substantially from this behavior. Figure $3.10 \mathrm{p}$ shows the peak $\omega_{z}$ versus the peak $\operatorname{div}_{h}$ from LCT. In this case, the relationship is less clear, considering the large vertical error bars. A constant ratio $\omega_{z} / \mathrm{div}_{h}$ is (at least by eye) consistent with the measurements. However, for outflows $\omega_{z}$ might also be constant. We note that the fit lines for the travel times in Fig. 3.10 have almost the same slopes for outflows and inflows, whereas in the case of LCT $\operatorname{div}_{h}$ and $\omega_{z}$ the slope for the inflows is much steeper than for the outflows. This is consistent with Fig. 3.7, where $\omega_{z}$ was shown to be twice as strong in the inflows as in the outflows, whereas the velocities $v^{\text {ac }}$ are of similar magnitude (not just for TD, but also for LCT). As discussed in Sects. 3.4.2 and 3.4.3, the velocities $v^{\text {ac }}$ do not directly measure the vorticity at a given position, but rather a spatial average.

In general, we can conclude that a selection bias in favor of stronger or weaker supergranules probably does not affect the measured ratio of vertical vorticity to horizontal divergence.

\subsubsection{Comparison of SDO/HMI and SOHO/MDI}

While the results for the average supergranule were obtained using different methods (TD and LCT) and image types (Dopplergrams and intensity images), they are all based on the same instrument, HMI. It is thus useful to compare the HMI results to $v^{\text {ac }}$ maps that have been measured from independent MDI data. Since MDI cannot sufficiently resolve granules at higher latitudes to successfully perform LCT, however, we only discuss TD.

In contrast to HMI, the correction for geometric center-to-limb systematics is not sufficient for MDI. For example, for f-mode TD at $40^{\circ}$ latitude, the central peak structure appears elongated (see Fig. 3.15p. Nevertheless, the $v^{\text {ac }}$ values at the origin are remarkably similar for HMI and MDI. At the average outflow, we measure $(-11.1 \pm 0.4) \mathrm{m} \mathrm{s}^{-1}$ (HMI) versus $(-10.1 \pm 0.8) \mathrm{m} \mathrm{s}^{-1}$ (MDI) for f modes and $(-7.7 \pm 0.3) \mathrm{m} \mathrm{s}^{-1}$ compared to $(-6.5 \pm 0.8) \mathrm{m} \mathrm{s}^{-1}$ for $\mathrm{p}_{1}$ modes.

For inflows, the MDI $v^{\text {ac }}$ maps compare to HMI in the same manner, with MDI being slightly weaker than HMI. The flow magnitudes for HMI and MDI at the origin after correction are $(8.9 \pm 0.4) \mathrm{m} \mathrm{s}^{-1}$ versus $(7.2 \pm 1.0) \mathrm{m} \mathrm{s}^{-1}$ for $\mathrm{f}$ modes and $(6.1 \pm 0.3) \mathrm{m} \mathrm{s}^{-1}$ compared to $(4.2 \pm 0.5) \mathrm{m} \mathrm{s}^{-1}$ for $\mathrm{p}_{1}$ modes. We note that the noise background is stronger for MDI. This is, however, not surprising, since only about half the number of Dopplergrams (compared to HMI) have been used to produce these maps.

The latitude dependence of $v^{\text {ac }}$ at the origin for MDI is qualitatively comparable with HMI (see Fig. 3.11). We measure a zero-crossing and sign change of $v^{\text {ac }}$ at the equator, both for the average supergranule outflow and inflow regions. However, the $v^{\text {ac }}$ magnitudes are systematically smaller for MDI. This difference increases farther away from the equator. It is especially dramatic for $\mathrm{f}$ modes at $\pm 60^{\circ}$ latitude. Whereas $v^{\text {ac }}$ reaches values between 10 and $12 \mathrm{~m} \mathrm{~s}^{-1}$ at these latitudes in HMI, for MDI the velocity magnitudes are below $5 \mathrm{~m} \mathrm{~s}^{-1}$. This is probably connected to the lower spatial resolution of MDI, which results in a larger impact of geometrical foreshortening effects at high latitudes compared 


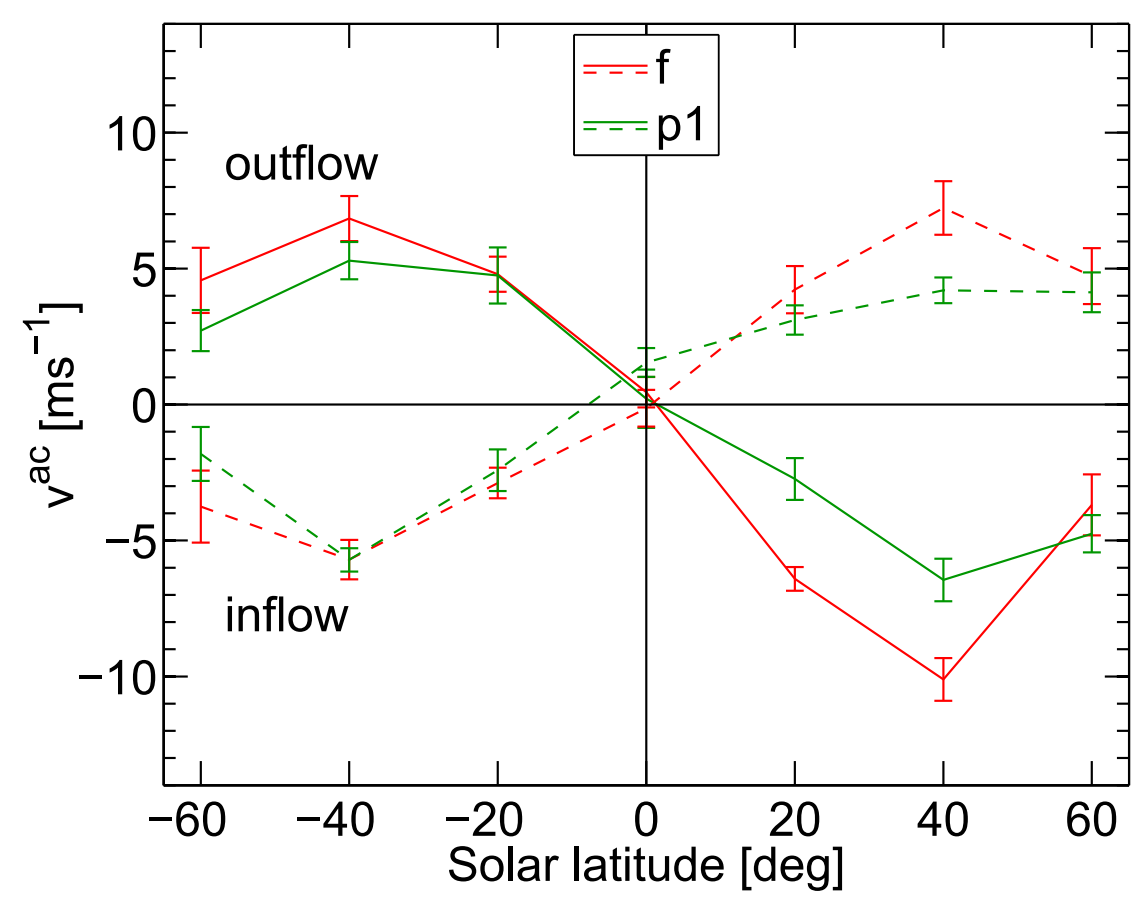

Figure 3.11: Velocities $v^{\text {ac }}$ for the average supergranule measured at the origin at different solar latitudes from MDI TD (f modes and $\mathrm{p}_{1}$ modes). Solid lines are for the average supergranule outflow region, dashed lines for the average supergranule inflow region. The error bars were computed by dividing the 177 datasets into eight parts.

to HMI.

While MDI clearly does not perform as well as HMI, the agreement with HMI at the origin gives reason to believe that MDI $v^{\text {ac }}$ measurements are still useful. This would be especially interesting for long-term studies of the solar cycle dependence since continuous data reaching back to 1996 could be used.

\subsection{Differences between outflow and inflow regions}

The differences between the average supergranule outflow and inflow regions as measured from LCT in HMI data are summarized in Fig. 3.12. The arrows show the horizontal velocity magnitudes and directions at $40^{\circ}$ latitude. The flows are dominated by the radial velocity component. For direct comparison, the filled contours give the vertical vorticity $\omega_{z}$ of the flows. In the average outflow region (Fig. 3.12 ), the vorticity shows a broad plateau region (FWHM about $13 \mathrm{Mm}$ ). The region of negative vorticity is surrounded by a ring of positive vorticity with a diameter of about $30 \mathrm{Mm}$.

In contrast, the vorticity in the average inflow region (Fig. $3.12 \mathrm{p}$ ) falls off rapidly from its narrow center (FWHM $8 \mathrm{Mm}$ ). We note that the FWHM of the vorticity peak is smaller than for the divergence peak (about $10 \mathrm{Mm}$ ) but still larger than the FWHM of the LCT correlation measurements (roughly $3 \mathrm{Mm}$ ). The peak vorticity magnitude is about twice the value of the outflow region (about $8 \times 10^{-6} \mathrm{~s}^{-1}$ anticlockwise compared to $4 \times 10^{-6} \mathrm{~s}^{-1}$ clockwise). As it is for the average outflow region, the central vorticity structure in the inflow region is surrounded by a ring of vorticity with opposite sign. The 


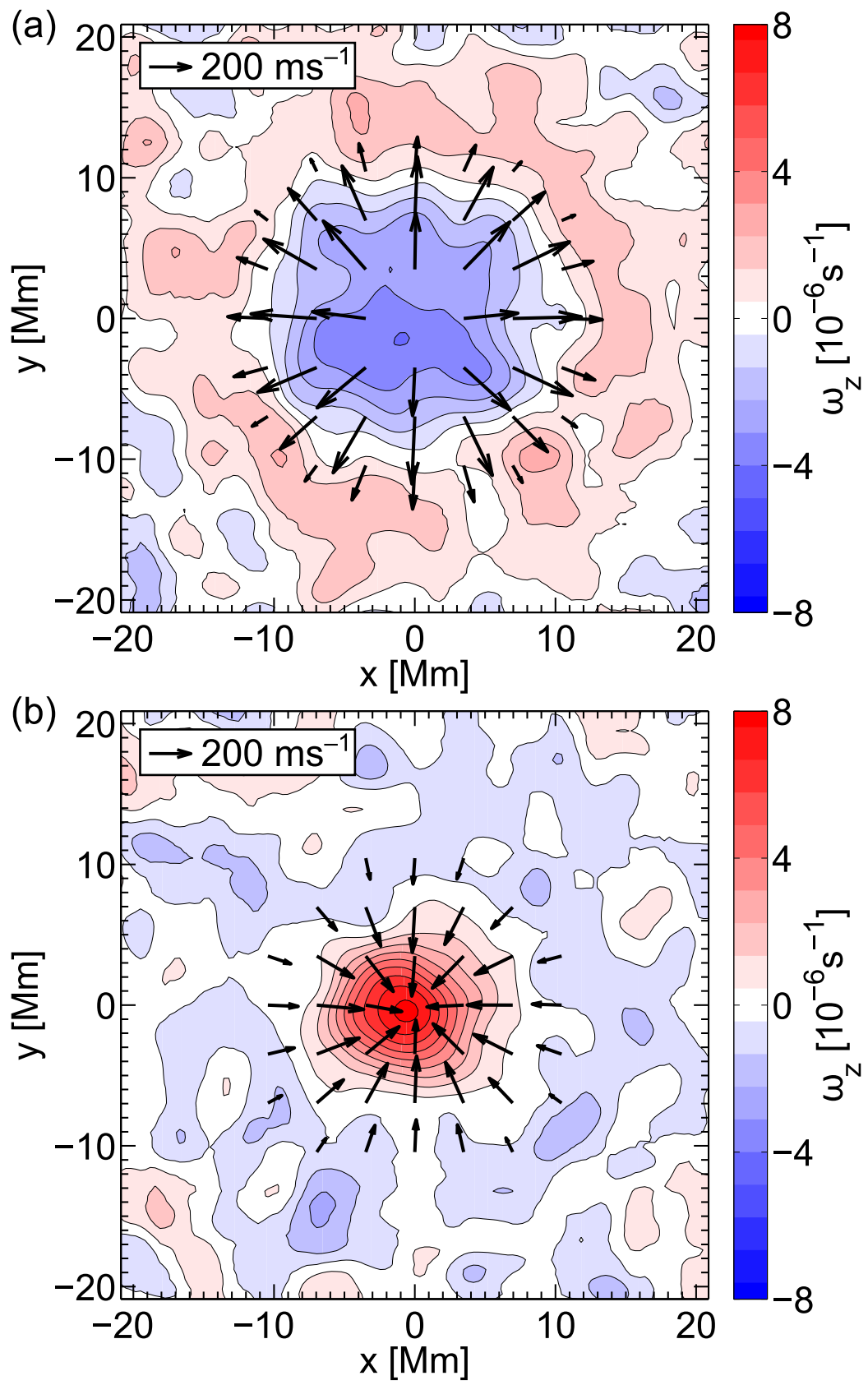

Figure 3.12: LCT horizontal velocity (black arrows) and vertical vorticity $\omega_{z}$ (filled contours) for the average supergranule at solar latitude $40^{\circ}$. a) Average outflow region. b) Average inflow region. Arrows for velocity magnitudes less than $60 \mathrm{~m} \mathrm{~s}^{-1}$ are omitted.

vorticity magnitude in the ring appears to be smaller than in Fig. 3.12 a.

These differences in the vortex structures of outflow and inflow regions are visible at all latitudes (except at the equator, where we measure no net vorticity). The FWHM of the peak structures as well as the ratio of the peak vorticities (between outflow and inflow regions) are constant over the entire observed latitude range. Such differences do not appear in maps of the horizontal divergence $\operatorname{div}_{h}$ (the FWHM is about $10 \mathrm{Mm}$ in both outflow and inflow regions). 
Differences in the vorticity strength between regions of divergent and convergent flows have also been reported by other authors who studied the statistics of vortices in solar convection. Wang et al. (1995) found, on granular scales, the root mean square of $\omega_{z}$ to be slightly higher in inflow regions. Pötzi \& Brandt (2007) observed that vortices are strongly connected to sinks at mesogranular scales. Concentration of fluid vorticity in inflows has also been found in simulations of solar convection (e.g., Stein \& Nordlund 1998, on granulation scale). However, the authors did not find any preferred sign of $\omega_{z}$. The increased vorticity strength in inflows might be a manifestation of the "bathtub effect" (Nordlund 1985). In that scenario, initially weak vorticity becomes amplified in inflows due to angular momentum conservation. In the downflows that are associated with the horizontal inflows because of mass conservation, the vortex diameter is reduced since the density rapidly increases with depth. This further enhances the vorticity.

\subsection{Radial and tangential velocities versus radial distance}

We now look in greater detail at the isotropic part of the horizontal flow profile of the average supergranule. Figs. $3.13 \mathrm{a}$ and $\mathrm{b}$ show the azimuthal averages of $v_{r}$ and $v_{t}$ around both the average supergranule outflow and inflow centers as a function of horizontal distance $r$ to the outflow/inflow center at $40^{\circ}$ latitude. In both cases, the magnitude of $v_{r}$ increases from the outflow/inflow center until it reaches a peak velocity (which we call $v_{r}^{\max }$ ) of slightly more than $300 \mathrm{~m} \mathrm{~s}^{-1}$ and $-200 \mathrm{~m} \mathrm{~s}^{-1}$, respectively at $r=7 \mathrm{Mm}$. The flow magnitudes then decrease and $v_{r}$ switches sign at a distance of about $14 \mathrm{Mm}$, marking the edge of the average inflow/outflow region. In general, the $v_{r}$ curves for outflow and inflow regions are similar except for the difference in flow magnitude.

The tangential velocity $v_{t}$, on the other hand, exhibits similar peak velocities for outflow and inflow regions (both $\left|v_{t}^{\max }\right| \approx 12 \mathrm{~m} \mathrm{~s}^{-1}$ ) but has opposite signs and reaches these peaks at different distances. The peak magnitude $v_{t}^{\max }$ is about 26 times smaller than $v_{r}^{\max }$ in the outflow region and 18 times smaller in the inflow region. In the outflow region, the peak is located at $r=9 \mathrm{Mm}$, whereas it lies at $r=5 \mathrm{Mm}$ around the average inflow center. Despite the different peak locations, $v_{t}$ switches sign at a distance of about $17 \mathrm{Mm}$ around the outflow and the inflow centers.

The different peak locations of $v_{t}$ possibly explain why the magnitude ratio of $v^{\text {ac }}$ between the average supergranule inflow and outflow region is smaller than one. The $v^{\text {ac }}$ measurements are especially sensitive to $v_{t}$ at $r=10 \mathrm{Mm}$ (the annulus radius). At this distance, we have $v_{t}=10 \mathrm{~m} \mathrm{~s}^{-1}$ around outflow centers, but $v_{t}=-7 \mathrm{~m} \mathrm{~s}^{-1}$ around inflow centers, yielding a factor of -0.7 . This agrees well with the ratio of the slopes for LCT $v^{\text {ac }}$ in Fig. 3.7 that we discussed in Sect. 3.4.3

Measuring the peak values of $v_{r}$ and $v_{t}$ at all latitudes except the equator leads to the following approximate relations:

$$
\begin{array}{ll}
v_{t}^{\max }=(-0.059 \pm 0.001) \frac{\Omega(\lambda) \sin \lambda}{\Omega_{0}} v_{r}^{\max } & \text { for outflows, } \\
v_{t}^{\max }=(-0.089 \pm 0.002) \frac{\Omega(\lambda) \sin \lambda}{\Omega_{0}} v_{r}^{\max } & \text { for inflows }
\end{array}
$$

where we used the differential rotation model from Snodgrass (1984) to compute $\Omega(\lambda)$, and $\Omega_{0}$ denotes the rotation rate at the equator. The coefficients $b_{\text {out }}:=-0.059 \pm 0.001$ and 
3 Paper II: Spatially resolved vertical vorticity in solar supergranulation using helioseismology and local correlation tracking

$b_{\text {in }}:=-0.089 \pm 0.002$ are remarkably constant over the whole latitude range from $-60^{\circ}$ to $60^{\circ}$, although $v_{r}^{\max }$ decreases from the equator $\left(335 \mathrm{~m} \mathrm{~s}^{-1}\right.$ for outflows and $-237 \mathrm{~m} \mathrm{~s}^{-1}$ for inflows) toward high latitudes (e.g., $272 \mathrm{~m} \mathrm{~s}^{-1}$ for outflows and $-188 \mathrm{~m} \mathrm{~s}^{-1}$ for inflows at $60^{\circ}$ north). The same trend is observed for measuring $v_{r}^{\max }$ west and east off disk center (e.g., $272 \mathrm{~m} \mathrm{~s}^{-1}$ for outflows and $-187 \mathrm{~m} \mathrm{~s}^{-1}$ for inflows at $60^{\circ}$ west), suggesting that the decrease is a systematic center-to-limb effect. Since $b_{\text {out }}$ and $b_{\text {in }}$ are not affected, $v_{t}^{\max }$ is likely to suffer from the same systematic decrease as $v_{r}^{\max }$.

We can use Eqs. (3.6) and (3.7) to predict $v_{t}^{\max }$ for supergranules in the polar regions. Assuming $b_{\text {out }}$ and $b_{\text {in }}$ are independent of latitude even beyond $\lambda= \pm 60^{\circ}$ and employing $v_{r}^{\max }$ from the equator where center-to-limb effects are small, the average supergranule at the north pole should rotate with $v_{t}^{\max }=\left(-20 \mathrm{~m} \mathrm{~s}^{-1}\right) \times \Omega\left(90^{\circ}\right) / \Omega_{0}$ for outflows and $v_{t}^{\max }=\left(21 \mathrm{~m} \mathrm{~s}^{-1}\right) \times \Omega\left(90^{\circ}\right) / \Omega_{0}$ for inflows. At the south pole, merely the sign of $v_{t}^{\max }$ should change.

Various authors have proposed models to describe vortex flows (e.g., Taylor 1918, Veronis 1959, Simon \& Weiss 1997), introducing the (turbulent) kinematic viscosity $\eta$ as a parameter that influences the tangential velocity component $v_{t}(r)$. In the Veronis model, the tangential flow is given by

$$
v_{t}=-\frac{2 l^{2}}{\pi^{2}\left(4+l^{2} / d^{2}\right)} \frac{\Omega(\lambda) \sin \lambda}{\eta} v_{r},
$$

where $l$ and $d$ are the horizontal size and depth of the supergranule, respectively. This relationship is consistent with the measurements in Eqs. (3.6) and (3.7). We note, though, that the Veronis convection model does not include turbulence (beyond $\eta$ ) or stratification.

Taylor (1918) presented a simple model that describes the decay of a narrow isolated vortex with $v_{r}=0$ due to fluid viscosity. In this case, the tangential velocity component is

$$
v_{t}(r)=\frac{a r}{\eta t^{2}} \exp \left(-r^{2} / 4 \eta t\right)
$$

with a constant $a$ and the "age" of the vortex $t$. A least-squares fit of Eq. (3.9) to our measured curve $v_{t}(r)$ describes the $v_{t}$ profile for the average inflow surprisingly well. We can use $4 \eta t \approx 7.8 \mathrm{Mm}$ from the fit to obtain a crude estimate of the turbulent viscosity. By identifying the vortex age $t$ with the supergranule lifetime ( $\sim 1$ day), we get $\eta \sim(1$ day $/ t) \times$ $180 \mathrm{~km}^{2} \mathrm{~s}^{-1}$. This is similar to values from the literature. For example, Duvall \& Gizon (2000) and Simon \& Weiss (1997) obtained $\eta=250 \mathrm{~km}^{2} \mathrm{~s}^{-1}$ using helioseismology and local correlation tracking of granules, respectively. The order of magnitude of our estimate for $\eta$ also agrees with previous measurements of the diffusion coefficient of small magnetic elements (Jafarzadeh et al. 2014, and references therein).

\subsection{Summary}

\subsubsection{Validation}

We have successfully measured the horizontal divergence and vertical vorticity of nearsurface flows in the Sun using different techniques (TD and LCT), as well as different instruments (HMI and MDI). Horizontal flow velocities from LCT compare well with 

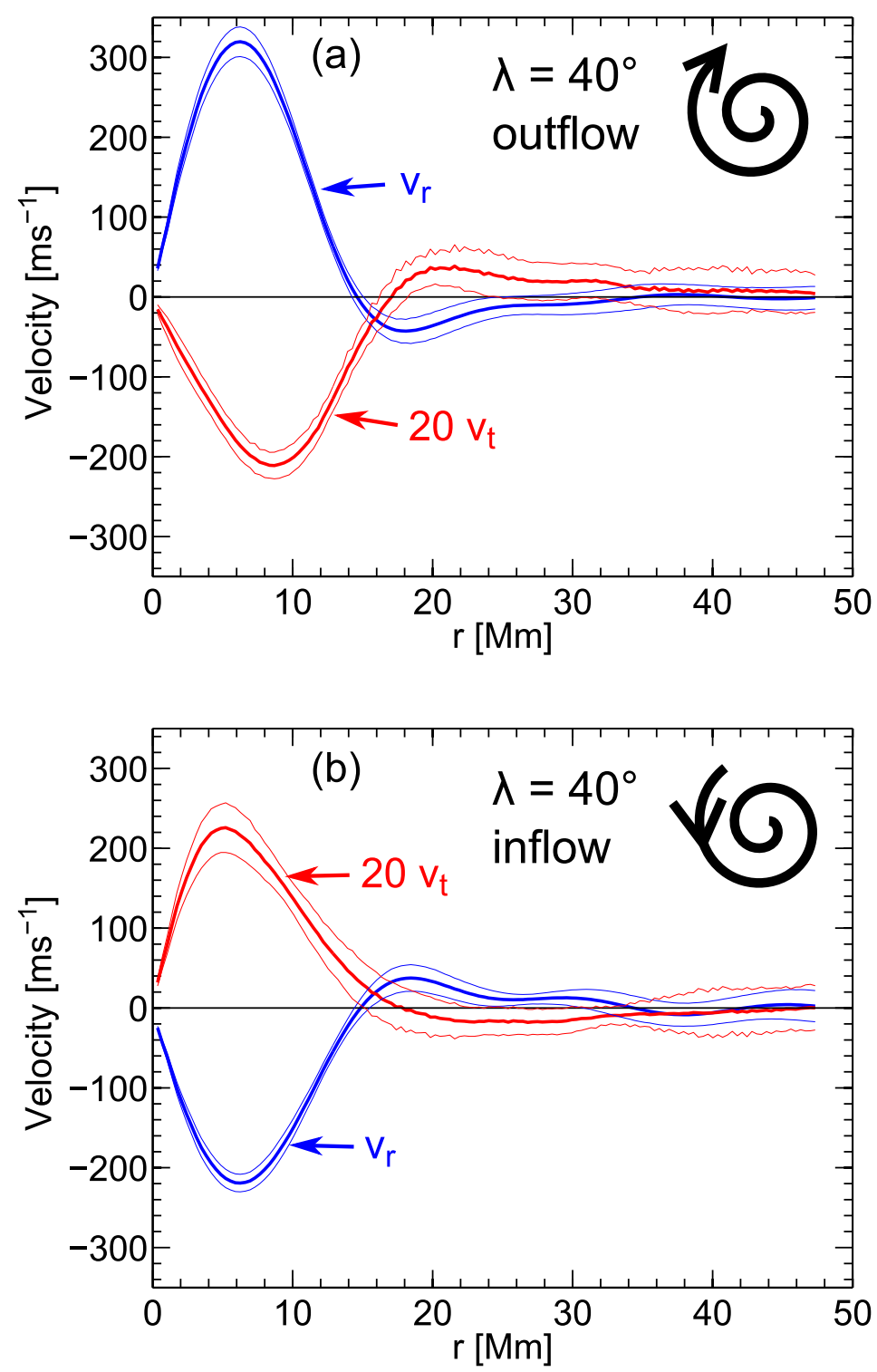

Figure 3.13: Azimuthally averaged horizontal velocities around the average supergranule outflow and inflow centers at solar latitude $40^{\circ}$. The measurements were obtained from LCT of granules in HMI intensity images. a) Horizontal velocities $v_{r}$ and $v_{t}$ around the average supergranule outflow center. The thin lines mark an estimate of the variability of the data as measured by dividing the 336 datasets into eight parts. For $v_{r}$, the $10 \sigma$ level is shown, for $v_{t}$ the $3 \sigma$ level. b) As a), but around the average supergranule inflow center. 
3 Paper II: Spatially resolved vertical vorticity in solar supergranulation using helioseismology and local correlation tracking

line-of-sight Dopplergrams (correlation coefficient 0.94). Horizontal divergence maps from TD and LCT are in excellent agreement for $8 \mathrm{~h}$ averaging (correlation coefficient 0.96 for $75 \lesssim k R_{\odot} \lesssim 175$ ). Vertical vorticity measurements from TD and LCT are highly correlated at large spatial scales (correlation coefficient larger than 0.7 for $k R_{\odot} \leq 100$ ).

We studied the average properties of supergranules by averaging over 3000 of them in latitude strips from $-60^{\circ}$ to $60^{\circ}$. The vertical vorticity maps as measured from HMI TD and HMI LCT for the average supergranule agree at low and mid latitudes. Above $\pm 40^{\circ}$ latitude, however, the LCT and TD results are different owing to geometrical centerto-limb systematic errors. After correcting for these errors using measurements at the equator away from the central meridian (cf. Zhao et al.2013), TD and LCT results agree well. For MDI, the TD maps are dominated by systematic errors even at low latitudes. Therefore, HMI is a significant improvement over MDI.

\subsubsection{Scientific results: spatial maps of vertical vorticity}

Our findings can be summarized as follows. The root mean square of the vertical vorticity in a map of size $\sim 180 \times 180 \mathrm{Mm}^{2}$ at the equator and $8 \mathrm{~h}$ averaging is about $15 \times 10^{-6} \mathrm{~s}^{-1}$ after low-pass filtering (power at scales $k R_{\odot}<300$ ).

After averaging over several thousand supergranules, the average outflow and inflow regions possess a net vertical vorticity (except at the equator). The latitudinal dependence of the vorticity magnitude is consistent with the action of the Coriolis force: $\omega_{z}(\lambda) \propto \Omega(\lambda) \sin \lambda / \Omega_{0}$. In the northern hemisphere, horizontal outflows are associated with clockwise motion, whereas inflows are associated with anticlockwise motion. In the southern hemisphere, the sense of rotation is reversed. This resembles the behavior of high and low pressure areas in the Earth's weather system (e.g., hurricanes).

Vortices in the average supergranular inflow regions are stronger and more localized than in outflow regions. For example, at $40^{\circ}$ latitude the vertical vorticity is $8 \times 10^{-6} \mathrm{~s}^{-1}$ anticlockwise in inflows versus $4 \times 10^{-6} \mathrm{~s}^{-1}$ clockwise in outflows, whereas the FWHM is $8 \mathrm{Mm}$ versus $13 \mathrm{Mm}$. The maximum tangential velocity in the average vortex is about $12 \mathrm{~m} \mathrm{~s}^{-1}$ at $\pm 40^{\circ}$ latitude, which is about 26 and 18 times smaller than the maximum radial flow component for outflow and inflow regions, respectively.

We have demonstrated the ability of TD and LCT to characterize rotating convection near the solar surface. This information can be used in the future to constrain models of turbulent transport mechanisms in the solar convection zone (see, e.g., Rüdiger et al. 2014). The azimuthally averaged velocity components $v_{r}$ and $v_{t}$ for supergranular outflows and inflows at various latitudes are available at the CDS.

\section{Acknowledgements}

JL, LG, and ACB designed research. JL performed research, analyzed data, and wrote the paper. JL and LG acknowledge research funding by Deutsche Forschungsgemeinschaft (DFG) under grant SFB 963/1 "Astrophysical flow instabilities and turbulence" (Project A1). The HMI data used are courtesy of NASA/SDO and the HMI science team. SOHO is a project of international cooperation between ESA and NASA. The data were processed at the German Data Center for SDO (GDC-SDO), funded by the German Aerospace Cen- 
ter (DLR). We thank J. Schou, T. L. Duvall Jr., and R. Cameron for useful discussions. We are grateful to R. Burston and H. Schunker for providing help with the data processing, especially the tracking and mapping. We used the workflow management system Pegasus (funded by The National Science Foundation under OCI SI2-SSI program grant \#1148515 and the OCI SDCI program grant \#0722019).

\subsection{Appendix: Ridge filters}

Prior to the travel-time measurements, the wavefield that is present in the Dopplergrams is filtered to select single ridges (the f modes or the $\mathrm{p}_{1}$ modes). The goal is to capture as much of the ridge power as possible, even if the waves are Doppler-shifted by flows. At the same time, we want to prevent power from neighboring ridges from leaking in and select as little background power as possible.

To construct the filter, we first measure the power spectra of the Dopplergrams at the equator and averaged over 60 days (59 days) of data in the case of HMI (MDI). After further azimuthal averaging, we identify the frequency $\omega_{\text {mode }}$ where the ridge maximum is located as a function of wavenumber $k$.

The filter is constructed for each $k$ as a plateau of width $2 \omega_{\delta}$ centered around the ridge maximum $\omega_{\text {mode }}$. The lower and upper boundaries of the plateau we call $\omega_{b}$ and $\omega_{c}$. Next to the plateau, we add a transition region of width $\omega_{\text {slope }}$, which consists of a raised cosine function that guides the filter from one to zero, symmetrically around $\omega_{\text {mode }}$. The lower and upper limits of the filter we call $\omega_{a}$ and $\omega_{d}$, respectively.

The plateau half-width $\omega_{\delta}$ consists of the following terms

$$
\omega_{\delta}(k)=\frac{\omega_{\Gamma}(k)}{2}+\omega_{v}(k)+\omega_{\text {const }}
$$

where $\omega_{\Gamma}(\mathrm{k})$ is the FWHM of the ridge (measured from the average power spectra), $\omega_{v}(k)=a k v_{\max }$ is the Doppler shift due to a hypothetical flow of magnitude $v_{\max }$ multiplied by a scale factor $a$, and $\omega_{\text {const }}$ is a constant term of small magnitude that broadens the filter predominantly at small wavenumbers.

The width of the transition region relative to the plateau width is

$$
\omega_{\text {slope }}=j \omega_{\delta}
$$

where $j$ is a unitless factor.

In addition, we restrict the filter to a range of wavenumbers. Above and below a $k$ interval, the filters are set to zero. The $k$ limits of the interval are chosen such that the ridge power is roughly twice the background power. Because $\omega_{\text {mode }}$ is a function of wavenumber, these limits can also be expressed as frequencies $\omega_{\min }$ and $\omega_{\max }$.

Table 3.2 lists the filter parameters we chose for the f-mode and $\mathrm{p}_{1}$-mode ridge filters that we use throughout the paper. We note that we use the same filters for all latitudes and longitudes. For the $p_{1}$ modes, we also list an alternative filter that we use to discuss the impact of the filter details on the travel-time measurements (see Appendix 3.10.3). 
3 Paper II: Spatially resolved vertical vorticity in solar supergranulation using helioseismology and local correlation tracking

Table 3.2: Parameters of the ridge filters that are used for the travel-time measurements in this paper (see text for details).

\begin{tabular}{cccc}
\hline \hline Parameter & \multicolumn{3}{c}{ Selected ridge } \\
& f modes & $\begin{array}{c}\mathrm{p}_{1} \text { modes } \\
\text { (regular) }\end{array}$ & $\begin{array}{c}\mathrm{p}_{1} \text { modes } \\
\text { (alternative) }\end{array}$ \\
\hline$\omega_{\min } / 2 \pi$ & $1.75 \mathrm{mHz}$ & $1.90 \mathrm{mHz}$ & $1.90 \mathrm{mHz}$ \\
$\omega_{\max } / 2 \pi$ & $5.00 \mathrm{mHz}$ & $5.40 \mathrm{mHz}$ & $5.00 \mathrm{mHz}$ \\
$\omega_{\text {const }} / 2 \pi$ & $0.025 \mathrm{mHz}$ & $0.025 \mathrm{mHz}$ & $0.030 \mathrm{mHz}$ \\
$v_{\max }$ & $500 \mathrm{~m} \mathrm{~s}^{-1}$ & $500 \mathrm{~m} \mathrm{~s}^{-1}$ & $500 \mathrm{~m} \mathrm{~s}^{-1}$ \\
$a$ & 1.0 & 1.0 & 2.0 \\
$j$ & 1.0 & 1.0 & 0.6 \\
\hline$\omega_{a} / 2 \pi$ & $2.50 \mathrm{mHz}$ & $3.10 \mathrm{mHz}$ & $3.03 \mathrm{mHz}$ \\
$\omega_{b} / 2 \pi$ & $2.67 \mathrm{mHz}$ & $3.29 \mathrm{mHz}$ & $3.20 \mathrm{mHz}$ \\
$\omega_{\operatorname{mode}} / 2 \pi$ & $2.84 \mathrm{mHz}$ & $3.48 \mathrm{mHz}$ & $3.48 \mathrm{mHz}$ \\
$\omega_{c} / 2 \pi$ & $3.01 \mathrm{mHz}$ & $3.67 \mathrm{mHz}$ & $3.77 \mathrm{mHz}$ \\
$\omega_{d} / 2 \pi$ & $3.18 \mathrm{mHz}$ & $3.86 \mathrm{mHz}$ & $3.94 \mathrm{mHz}$ \\
\hline
\end{tabular}

Notes. The lower part of the table gives the filter limits at $k R_{\odot}=800$. The filter limits for HMI and MDI are equivalent.

\subsection{Appendix: Conversion of travel times into flow veloc- ities}

Point-to-point travel times $\tau^{\mathrm{diff}}\left(\mathbf{r}_{1}, \mathbf{r}_{2}\right)$ are sensitive to flows in the direction of $\mathbf{r}_{1}-\mathbf{r}_{2}$. If the flow structure is known, travel times $\tau^{\text {diff }}$ can be predicted with the knowledge of sensitivity kernels. Conversely, the velocity field can be obtained from measured travel times by an inversion. Such inversions are, however, delicate, as they are, in general, ill-posed problems. A simple way to obtain rough estimates of the flow velocity while avoiding inversions is the multiplication of the travel times by a constant conversion factor. Such a conversion factor can be calculated by artificially adding the signature of a uniform flow of known magnitude and direction to Dopplergrams. The magnitude of the measured travel time divided by the input flow speed yields the conversion factor. In the following, we describe this process.

First, we create data cubes $\phi_{v}(\mathbf{r}, t)$ that have Doppler-shifted power spectra to mimic the effect of a flow $\mathbf{v}$ independent of position $\mathbf{r}$ and time $t$. The data cubes are based on the noise model by Gizon \& Birch (2004), so signatures from flows others than $\mathbf{v}$ are not present. Following the noise model, we construct in Fourier space $\phi_{v}(\mathbf{k}, \omega)=$ $\sqrt{\mathcal{P}_{v}(\mathbf{k}, \omega)} \mathcal{N}_{0,1}(\mathbf{k}, \omega)$. Here $\mathbf{k}$ is the horizontal wave vector; $\mathcal{P}_{v}$ is a Doppler-shifted power spectrum; and, at each $(\mathbf{k}, \omega), \mathcal{N}_{0,1}$ are independent complex Gaussian random variables with zero mean and unit variance. Employing $\mathcal{N}_{0,1}$ ensures that the values $\phi_{v}(\mathbf{k}, \omega)$ are uncorrelated, which means that there is no signal from wave scattering. We use $\mathcal{P}_{v}(\mathbf{k}, \omega)=$ $\mathcal{P}_{0}(\mathbf{k}, \omega-\delta \omega)$ based on an average power spectrum $\mathcal{P}_{0}$ that was measured from 60 days of HMI Dopplergrams (and 59 days of MDI Dopplergrams) at the solar equator. The 
quantity $\delta \omega=\mathbf{k} \cdot \mathbf{v}$ is the frequency shift due to a background flow $\mathbf{v}=\left(v_{x}, 0\right)$ that we add. We construct $8 \mathrm{~h}$ datasets $\phi_{v}(\mathbf{r}, t)$ for $v_{x}$ in the range between -1000 and $1000 \mathrm{~m} \mathrm{~s}^{-1}$ in steps of $100 \mathrm{~m} \mathrm{~s}^{-1}$. For each velocity value, we compute 10 realizations.

As a consistency check, we apply a second method for adding an artificial velocity signal to the HMI Dopplergram datasets. This procedure consists of tracking at an offset rate. The tracking parameters from Snodgrass (1984) are modified by a constant corresponding to a $v_{x}$ velocity of $-100 \mathrm{~m} \mathrm{~s}^{-1}$ and $100 \mathrm{~m} \mathrm{~s}^{-1}$, respectively. The tracking and mapping procedure is as for the regular HMI observations. We produce 112 such datacubes for each $v_{x}$ value at the solar equator.

For both methods, the $8 \mathrm{~h}$ datasets are ridge-filtered like the normally tracked Doppler observations (f modes and $\mathrm{p}_{1}$ modes). We measure travel times $\tau^{\text {diff }}$ in the $x$ direction with the pairs of measurement points separated by $10 \mathrm{Mm}$. This distance matches the separation in the $\tau^{\mathrm{ac}}$ measurements. The reference cross-covariance $C^{\text {ref }}$ is taken from the regularly tracked HMI (MDI) observations averaged over 60 days (59 days) of data at the solar equator. This ensures that the artificial flow signal is captured by the travel-time measurements.

The resulting $\tau^{\text {diff }}$ values averaged over maps and datasets are shown for HMI in Fig. 3.14. For both $\mathrm{f}$ and $\mathrm{p}_{1}$ modes, the travel times from offset tracking are systematically larger than for the Doppler-shifted power spectra by about 10 to $15 \%$. In general, the travel-time magnitudes are larger for the $\mathrm{f}$ modes than for the $\mathrm{p}_{1}$ modes for the same input velocity value. The relation between input velocity $v_{x}$ and output travel time $\tau^{\text {diff }}$ is linear only in a limited velocity range. Whereas this range spans from roughly $-700 \mathrm{~m} \mathrm{~s}^{-1}$ to $700 \mathrm{~m} \mathrm{~s}^{-1}$ for the $\mathrm{p}_{1}$ modes, it only reaches from $-200 \mathrm{~m} \mathrm{~s}^{-1}$ to $200 \mathrm{~m} \mathrm{~s}^{-1}$ for the $\mathrm{f}$ modes. For velocity magnitudes larger than $700 \mathrm{~m} \mathrm{~s}^{-1}$, the measured f-mode travel times even decrease. However, the supergranular motions that we analyze reach typical velocities of $\sim 300 \mathrm{~m} \mathrm{~s}^{-1}$, which is well below that regime.

We applied a least-squares fit to a polynomial of degree three to the $\tau^{\mathrm{diff}}$ measurements from Doppler-shifted cubes (pink curve):

$$
\tau_{x}^{\mathrm{diff}}\left(v_{x}\right)=h_{3} v_{x}^{3}+h_{2} v_{x}^{2}+h_{1} v_{x}+h_{0} .
$$

The linear term of the polynomial is shown for HMI as the red curve in Fig. 3.14. For the actual conversion, only the linear coefficient $h_{1}$ is used. We obtain $h_{1}=-0.178 \mathrm{~s}^{2} \mathrm{~m}^{-1}$ for the $f$ modes and $h_{1}=-0.090 \mathrm{~s}^{2} \mathrm{~m}^{-1}$ for the $\mathrm{p}_{1}$ modes. For comparison, the coefficients $h_{1}$ are listed for different distances in Table 3.3. The table also contains the coefficients for MDI. We convert travel times into velocities by multiplying the travel times by $1 / h_{1}$. The velocities obtained from converting $\tau^{\text {ac }}$ maps we call $v^{\text {ac }}$.

\subsection{Appendix: Systematic errors}

\subsubsection{Center-to-limb systematics}

At high latitudes, the original $v^{\text {ac }}$ and LCT $\omega_{z}$ maps for the average supergranule show strong deviations from the azimuthally symmetric peak-ring structures that are visible at low latitudes. Considering that the magnitude of $\tau^{\text {ac }}$ and $\omega_{z}$ is much smaller than the magnitude of $\tau^{\mathrm{oi}}$ and $\operatorname{div}_{h}$ at any latitude, it is possible that even a small anisotropy in 

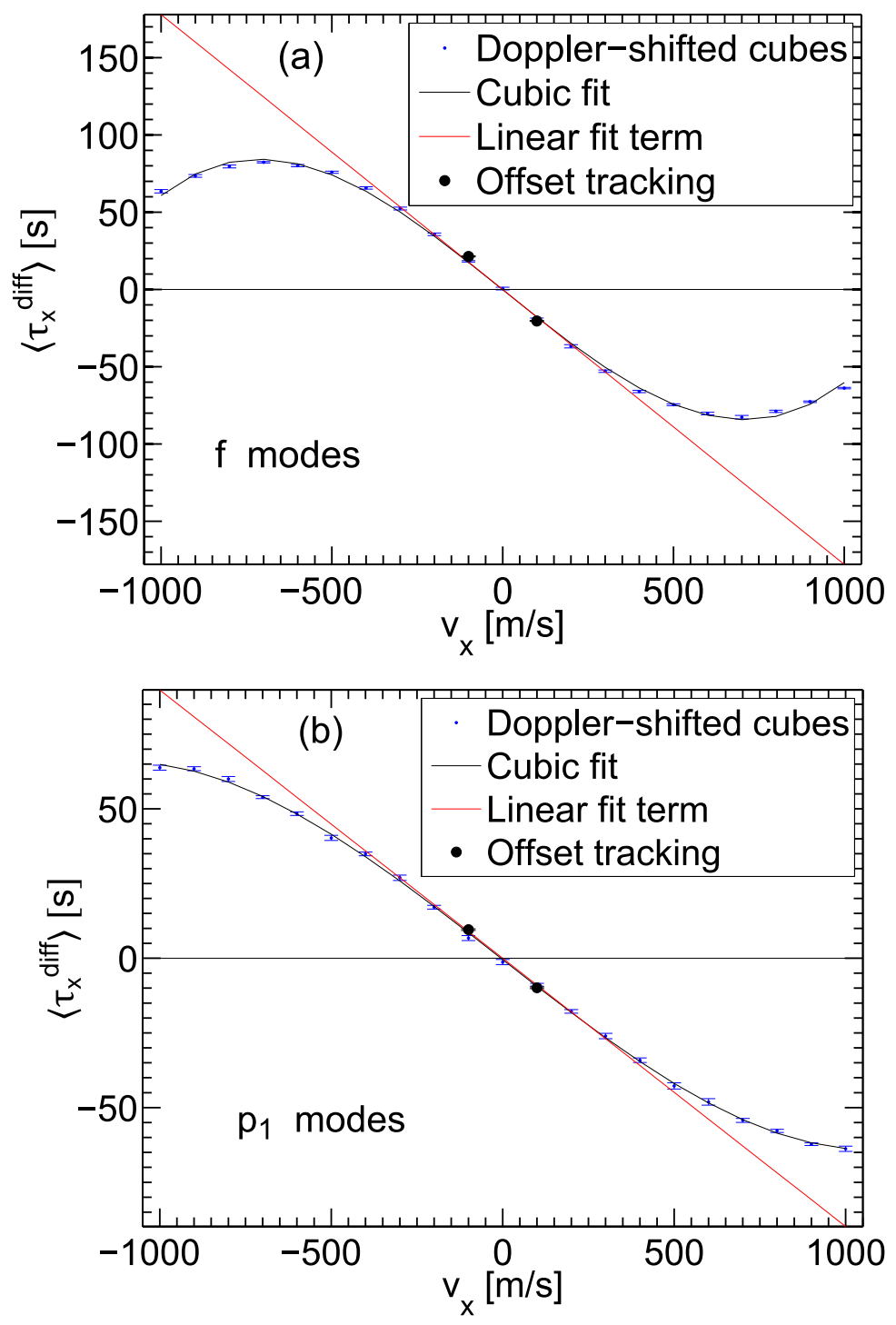

Figure 3.14: Point-to-point travel times from HMI Dopplergrams with artificial velocity signal. The point separation is $10 \mathrm{Mm}$ in the east-west direction. a) $\mathrm{f}$ modes. b) $\mathrm{p}_{1}$ modes. The blue dots give the travel times from Dopplergram series that were constructed using the noise model by Gizon \& Birch (2004). We applied a least-squares fit with a polynomial of degree three to the resulting data (black curves). The red curves show the linear term of the fit. For comparison, the black filled circles show travel times from HMI Dopplergrams that were tracked at an offset rate. 
Table 3.3: Coefficients $h_{1}$ of the cubic polynomial defined in Eq. (3.12) obtained from a least-squares fit.

\begin{tabular}{cccc}
\hline \hline Instrument & Distance & \multicolumn{2}{c}{$\begin{array}{c}\text { Value of coefficient } h_{1}\left[\mathrm{~s}^{2} \mathrm{~m}^{-1}\right] \text { for } \\
\text { f modes }\end{array}$} \\
& & $\mathrm{p}_{1}$ modes \\
\hline HMI & $5 \mathrm{Mm}$ & $-9.61 \times 10^{-2}$ & $-4.48 \times 10^{-2}$ \\
& $10 \mathrm{Mm}$ & $-1.78 \times 10^{-1}$ & $-8.98 \times 10^{-2}$ \\
& $15 \mathrm{Mm}$ & $-2.20 \times 10^{-1}$ & $-1.39 \times 10^{-1}$ \\
& $20 \mathrm{Mm}$ & $-2.15 \times 10^{-1}$ & $-1.71 \times 10^{-1}$ \\
\hline MDI & $5 \mathrm{Mm}$ & $-7.34 \times 10^{-2}$ & $-4.66 \times 10^{-2}$ \\
& $10 \mathrm{Mm}$ & $-1.58 \times 10^{-1}$ & $-8.91 \times 10^{-2}$ \\
& $15 \mathrm{Mm}$ & $-1.97 \times 10^{-1}$ & $-1.12 \times 10^{-1}$ \\
& $20 \mathrm{Mm}$ & $-2.43 \times 10^{-1}$ & $-1.58 \times 10^{-1}$ \\
\hline
\end{tabular}

Notes. The coefficient $h_{1}$ for a distance of $10 \mathrm{Mm}$ is used to convert measured travel times into flow velocities. For comparison, the coefficients for other distances are also given.

the divergent flow component of the average supergranule is picked up by the $v^{\mathrm{ac}}$ and $\omega_{z}$ measurements and added to the signal from the tangential flow component that we want to measure. Such anisotropies can arise from various origins. Among them are geometrical effects that depend on the distance to the disk center.

For TD measurements, the sensitivity kernels depend on the distance to the limb. At $60^{\circ}$ off disk center, $\tau^{\text {diff }}$ sensitivity kernels for measurements in the direction along the limb differ strongly from kernels for measurements in the center-to-limb direction (see, e.g., Jackiewicz et al. 2007, for a discussion). Additionally, there is a gradient of the root mean square travel time in the center-to-limb direction.

In the case of LCT, the shrinking Sun effect causes large-scale gradients of the horizontal velocity (of several hundred meters per second) pointing toward disk center (Lisle $\&$ Toomre 2004). This effect is presumably caused by insufficient resolution of the granules. Although HMI intensity and Doppler images have a pixel size of about $350 \mathrm{~km}$ at disk center, the point spread function has a FWHM of about twice that value. In Dopplergrams, the hot, bright, and broad upflows in the granule cores cause stronger blueshifts than the redshifts from the cooler, darker, and narrow downflows. Because of the insufficient resolution, the granules appear blueshifted as a whole. This blueshift adds to the blueshift of granules that move toward the observer (i.e., toward disk center), giving them a stronger signal in the Dopplergram. Lisle \& Toomre argue that LCT of Dopplergrams gives more weight to these granules than to those granules that move away from the observer. However, it is not clear what causes the shrinking Sun effect in LCT of intensity images. Fortunately, the shrinking Sun effect appears to be a predominantly large-scale and time-independent effect, so it can easily be removed from LCT velocity maps by subtracting a mean image.

Another problem is the foreshortening. Far away from the disk center, the granules are not as well resolved in the center-to-limb direction as in the perpendicular horizontal direction. This introduces a dependence of the measurement sensitivity on angle. We measure at $\pm 60^{\circ}$ latitude that the radial flow component $v_{r}$ of the average supergranule 
3 Paper II: Spatially resolved vertical vorticity in solar supergranulation using helioseismology and local correlation tracking
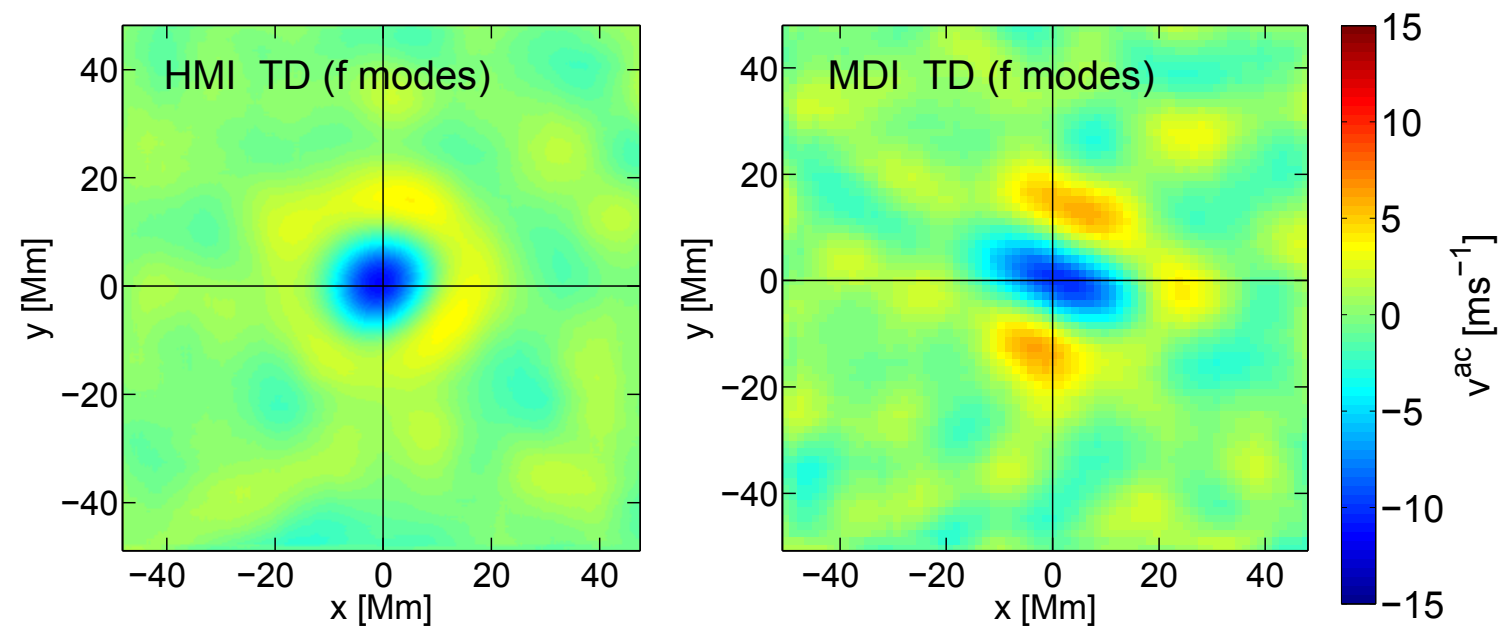

Figure 3.15: Circulation velocities $v^{\text {ac }}$ of the average supergranule outflow region at solar latitude $40^{\circ}$ derived from HMI and MDI Dopplergrams (after the correction for centerto-limb systematics). The velocity maps were obtained by applying the respective conversion factors from Appendix 3.9 to the travel times $\tau^{\text {ac }}$. The limits of the colorscale are arbitrarily set to $\pm 15 \mathrm{~m} \mathrm{~s}^{-1}$.

is weaker by 15 to $20 \%$ in the center-to-limb direction compared to the perpendicular direction. This corresponds to a maximum velocity difference of about $50 \mathrm{~m} \mathrm{~s}^{-1}$ for outflows and $30 \mathrm{~m} \mathrm{~s}^{-1}$ for inflows. At $40^{\circ}$ latitude, in contrast, this difference is less than $2 \%\left(6 \mathrm{~m} \mathrm{~s}^{-1}\right)$.

\subsubsection{MDI instrumental systematics}

Whereas for HMI the removal of geometrical center-to-limb effects results in similar $v^{\text {ac }}$ peak structures in the supergranule outflow regions in the whole latitude range from $-60^{\circ}$ to $60^{\circ}$, for MDI the peak structures appear asymmetric and distorted even after the correction. An example for f-mode TD at $40^{\circ}$ latitude is shown in Fig. 3.15. Even at disk center where geometrical effects should not play a role, there are visible systematic features (that do not appear for HMI, cf. Fig. 3.6). This is probably due to instrumental effects that are specific to MDI (see, e.g., Korzennik et al. 2004, for a discussion of instrumental errors in MDI).

\subsubsection{Selection of filter and $\tau^{\text {ac }}$ geometry parameters}

We note that the $v^{\text {ac }}$ velocity results for TD depend on the details of the ridge filter as well as the geometry parameters $(\Delta, n)$ of the $\tau^{\text {ac }}$ measurements.

To give an idea of this, we construct an alternative $\mathrm{p}_{1}$ ridge filter with slightly different width parameters (see Appendix 3.8). Additionally, we select four other combinations $(\Delta, n)$ of $\tau^{\text {ac }}$ measurements that preserve the annulus radius $R$, so that $R$ is within $(10.0 \pm$ $0.5) \mathrm{Mm}$ for all the combinations $(\Delta, n)$. As we did for the standard combination $(\Delta=$ $10 \mathrm{Mm}, n=6$ ), we use four different angles $\beta$ for each additional combination.

For all these combinations and both the standard and modified $\mathrm{p}_{1}$ filters, we calcu- 

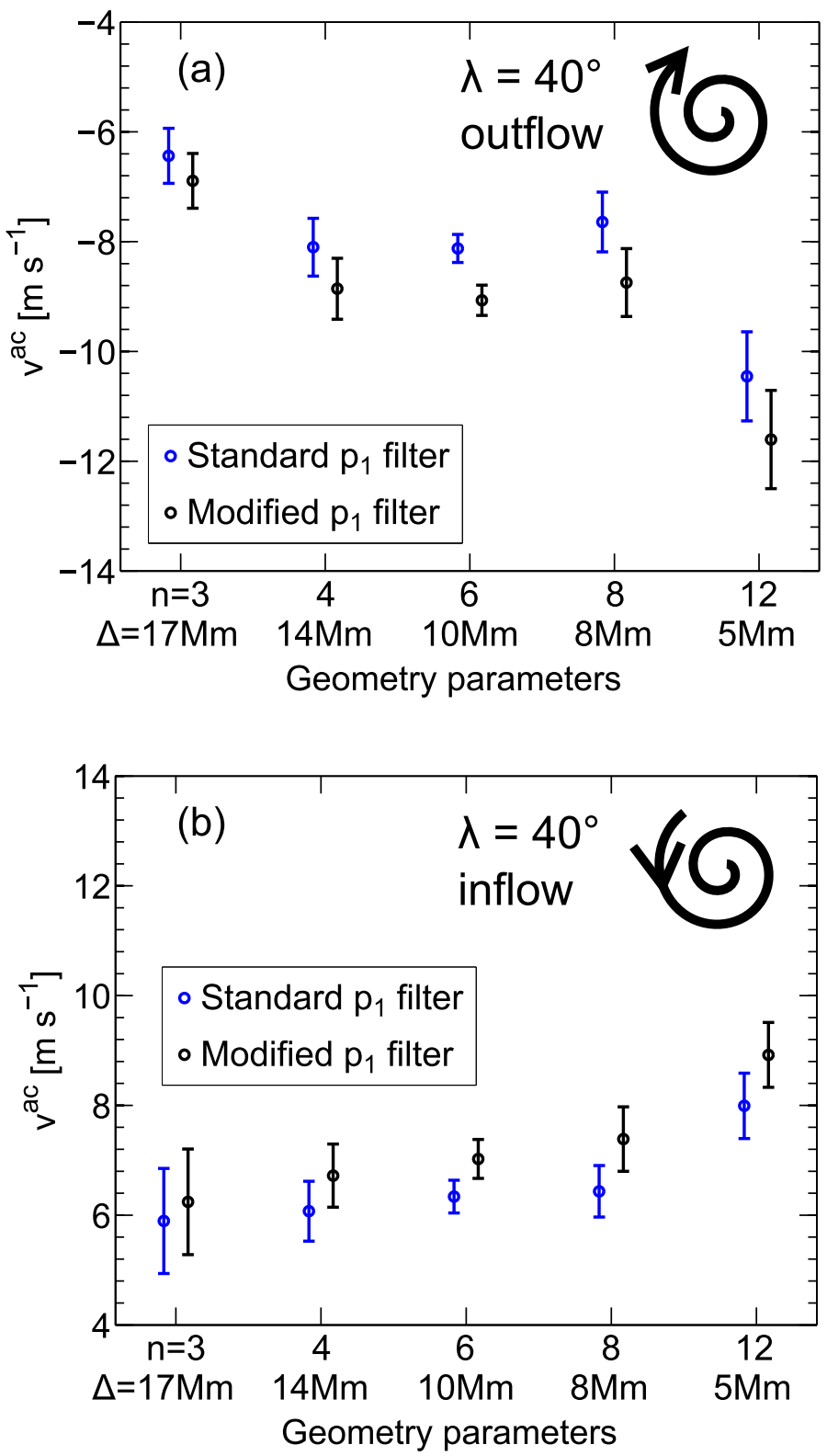

Figure 3.16: Peak $v^{\text {ac }}$ values for $\mathrm{p}_{1}$ modes using different parameter combinations $(\Delta, n)$ for the average supergranule at solar latitude $40^{\circ}$. a) In the average outflow region. b) In the average inflow region. The blue symbols give the results for the $\mathrm{p}_{1}$ ridge filter that has been used throughout this paper. For the results in black, an alternative $\mathrm{p}_{1}$ ridge filter with slightly different parameters was used (see text for details). The error bars were computed as in Fig. 3.7. The annulus radii corresponding to the various combinations $(\Delta, n)$ are all within $(10.0 \pm 0.5) \mathrm{Mm}$. 
3 Paper II: Spatially resolved vertical vorticity in solar supergranulation using helioseismology and local correlation tracking

lated $v^{\text {ac }}$ for the average supergranule at $40^{\circ}$ latitude. The resulting peak velocities are shown in Fig. 3.16 for both inflow and outflow regions. We did not apply the center-tolimb correction since it only has a weak influence on the peak velocity magnitude at $40^{\circ}$ latitude.

Evidently, the modified $\mathrm{p}_{1}$ filter results in systematically larger $v^{\mathrm{ac}}$ amplitudes. The difference with respect to the standard filter increases with decreasing $\Delta$. For $\Delta=10 \mathrm{Mm}$ and $n=6$, it is about $10 \%$. This is qualitatively in line with Duvall \& Hanasoge (2013). Using phase-speed filters, Duvall \& Hanasoge observed that the strength of the traveltime signal from supergranulation is strongly dependent on the filter width. This shows that one should be careful when comparing absolute velocities from TD and LCT. For more reliable velocity values, an inversion of $\tau^{\mathrm{oi}}$ and $\tau^{\mathrm{ac}}$ maps would be needed.

The comparison of different combinations $(\Delta, n)$ for the same filter shows that for $n=$ 4,6 , and 8 the $v^{\text {ac }}$ amplitudes are similar, so selecting the combination $(\Delta=10 \mathrm{Mm}, n=$ 6 ), as we did for most of this work, appears justified. Decreasing $\Delta$ to about $5 \mathrm{Mm}$ changes the peak $v^{\text {ac }}$ values. A possible reason is that $\Delta$ in this case becomes comparable to the wavelength of the oscillations, so it is harder to distinguish between flows in opposite directions. For small $n$, on the other hand, the measurement geometry deviates strongly from a circular contour. This might explain the deviations in $v^{\text {ac }}$ for $n=3$. 


\title{
4 Paper III: Anisotropy of the solar network magnetic field around the average supergranule
}

\begin{abstract}
Supergranules in the quiet Sun are outlined by a web-like structure of enhanced magnetic field strength, the so-called magnetic network. We aim to map the magnetic network field around the average supergranule near disk center. We use observations of the line-of-sight component of the magnetic field from the Helioseismic and Magnetic Imager (HMI) onboard the Solar Dynamics Observatory (SDO). The average supergranule is constructed by coaligning and averaging over 3000 individual supergranules. We determine the positions of the supergranules with an image segmentation algorithm that we apply to maps of the horizontal flow divergence measured using time-distance helioseismology. In the center of the average supergranule, the magnetic (intranetwork) field is weaker by about 2.2 Gauss than the background value (3.5 Gauss), whereas it is enhanced in the surrounding ring of horizontal inflows (by about 0.6 Gauss on average). We find that this network field is significantly stronger west (prograde) of the average supergranule than in the east (by about 0.3 Gauss). With time-distance helioseismology, we find a similar anisotropy. The observed anisotropy of the magnetic field adds to the mysterious dynamical properties of solar supergranulation. 1$]$
\end{abstract}

\subsection{Introduction}

Solar supergranules are surrounded by the network magnetic field that can be observed, for instance, in Ca II K emission lines in the solar chromosphere (e.g., Simon \& Leighton 1964). The network field is built up through the advection of magnetic field by supergranular flows (e.g., Rieutord \& Rincon 2010). Beyond this, however, not much is known about the dynamical interaction of supergranulation and the network field, and the dynamics of supergranulation itself is not understood (e.g., Gizon et al. 2003, Rieutord \& Rincon 2010).

\footnotetext{
${ }^{1}$ This chapter reproduces the article Anisotropy of the solar network magnetic field around the average supergranule by J. Langfellner, L. Gizon, and A. C. Birch, published in Astronomy \& Astrophysics 579, L7 (2015), DOI 10.1051/0004-6361/201526422. Reproduced with permission from Astronomy \& Astrophysics, (C) ESO. Contributions: JL, LG, and ACB designed research. JL performed research, analyzed data, and wrote the paper.
} 
4 Paper III: Anisotropy of the solar network magnetic field around the average supergranule

In this letter, we present photospheric maps of the magnetic field of the average supergranule using data from the Helioseismic and Magnetic Imager (HMI) (Schou et al.2012) onboard the Solar Dynamics Observatory (SDO) at full resolution (about $1 \mathrm{arcsec}$ ). The average supergranule is constructed as an ensemble average of individual supergranules that are identified in maps of the horizontal flow divergence from time-distance helioseismology (Duvall \& Gizon 2000).

\subsection{Observations and data processing}

We tracked $12^{\circ} \times 12^{\circ}$ patches of HMI line-of-sight magnetograms near disk center from 1 May through 28 August 2010, using a tracking rotation rate of $14.33^{\circ}$ per day. The patches were remapped using Postel's projection and a spatial sampling of $0.348 \mathrm{Mm}$. The temporal cadence is $45 \mathrm{~s}$. The $24 \mathrm{~h}$ datacubes are centered around the central meridian. Each $24 \mathrm{~h}$ datacube is divided into three $8 \mathrm{~h}$ chunks. The line-of-sight magnetic field $B_{\mathrm{LOS}}$ near disk center consists almost solely of the vertical magnetic field component.

In the same way and for the same patches, we tracked and remapped HMI line-of-sight Dopplergrams. We apply f-mode time-distance helioseismology to the $8 \mathrm{~h}$ datacubes to infer the horizontal divergence of the flow field (Langfellner et al. 2014, 2015). This is achieved by computing the temporal cross-correlation between each point and a surrounding annulus (10 Mm radius) and measuring the outward minus inward travel times. Additionally, we computed the average of inward and outward travel times, i.e. the mean travel times. The mean travel times are known to be sensitive to the magnetic field (Duvall et al. 2006).

From the divergence maps, we identified the supergranule boundaries using the image segmentation algorithm from Hirzberger et al. (2008). The lefthand panel of Fig. 4.1 shows an example $8 \mathrm{~h}$ divergence map with magnetic field contours overlaid and also shows the centers of supergranules (at maximum divergence). Following Duvall \& Birch (2010) and Langfellner et al. (2015), we construct an average supergranule by shifting and averaging over all the individual supergranules (about 3000 ). In the righthand panel of Fig. 4.1, we show the horizontal divergence of the average supergranule. The average outflow is surrounded by a ring of inflows with a radius of $15 \mathrm{Mm}$.

\subsection{Results}

\subsubsection{Magnetic field of the average supergranule near disk center}

By using the coordinates of the supergranules determined in the divergence maps, we construct a magnetic field map for the average supergranule (left panel of Fig. 4.2). The quantity we average over is the absolute value of $B_{\mathrm{LOS}}$, where the absolute value has been taken after averaging $B_{\mathrm{LOS}}$ over the length of a datacube $(8 \mathrm{~h})$. We use $B$ to denote this quantity. The rapidly varying small-scale magnetic field is substantially suppressed in this analysis. The spatially averaged $B$ has a value of $\bar{B}=3.52$ Gauss. In the center of the average supergranule, the magnetic field is weaker than $\bar{B}$ by about 2.2 Gauss. In the inflow region, on the other hand, the (network) magnetic field is stronger than the average value by up to roughly 1 Gauss. Surprisingly, the network field of the average 

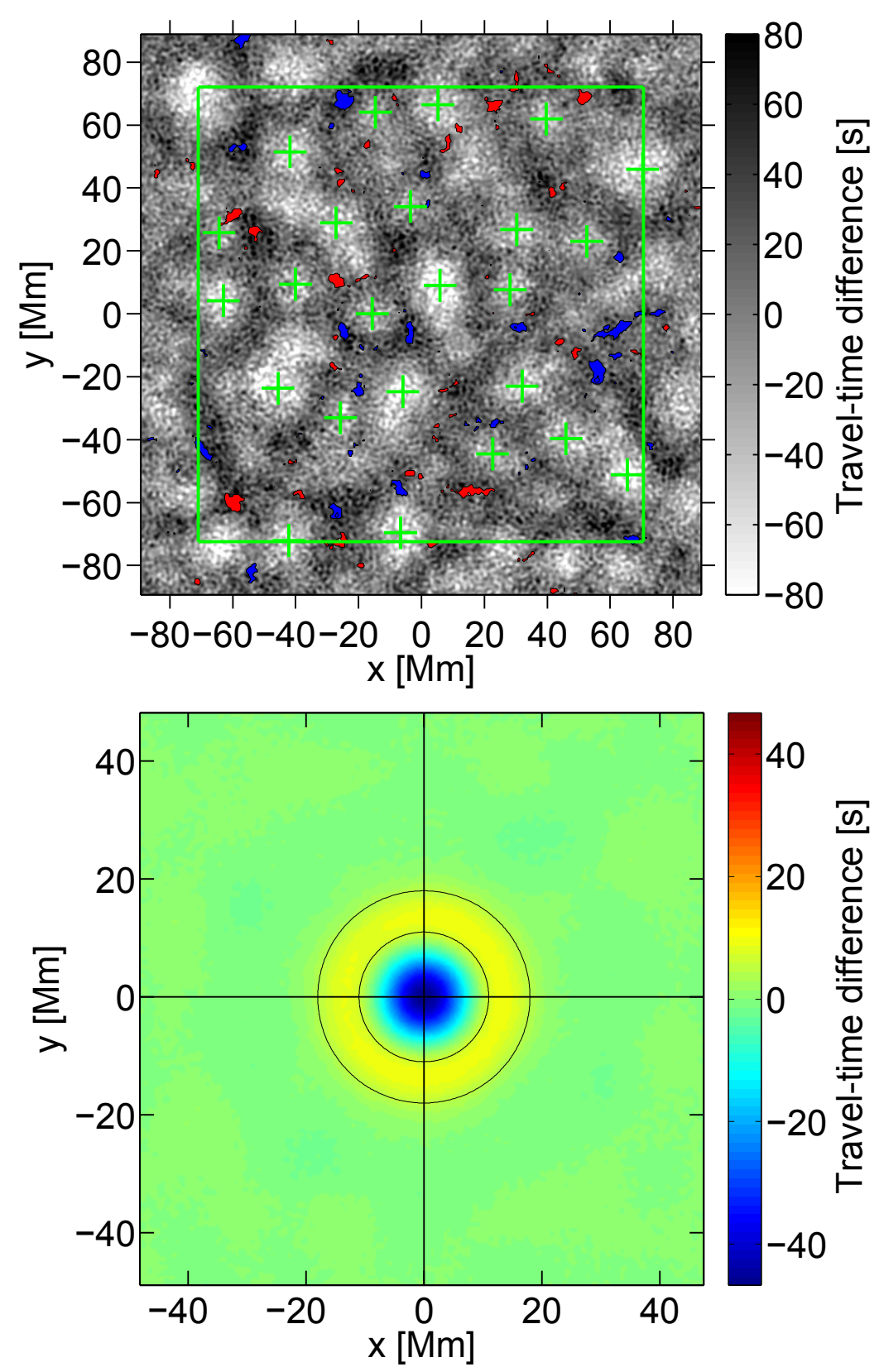

Figure 4.1: Top: Positions (green crosses) of centers of supergranules identified near disk center on 2 May 2010. Supergranules that are outside of the green frame have been discarded. The grayscale image shows the f-mode travel-time differences (white is for outflows, black for inflows) using $8 \mathrm{~h}$ of SDO/HMI observations. The colorbar is truncated in the range between -80 and $80 \mathrm{~s}$. The filled contours denote areas where the HMI line-of-sight magnetic field averaged over $8 \mathrm{~h}$ exceeds 20 Gauss in absolute value (red is positive field strength, blue is negative). Bottom: Travel-time map for the average supergranule, where negative (positive) values correspond to horizontal outflows (inflows). The black circles have radii of 11 and $18 \mathrm{Mm}$.

supergranule is stronger in the west (in the prograde direction) than in the east. This is a statistically highly significant result (as we show later). 
4 Paper III: Anisotropy of the solar network magnetic field around the average supergranule
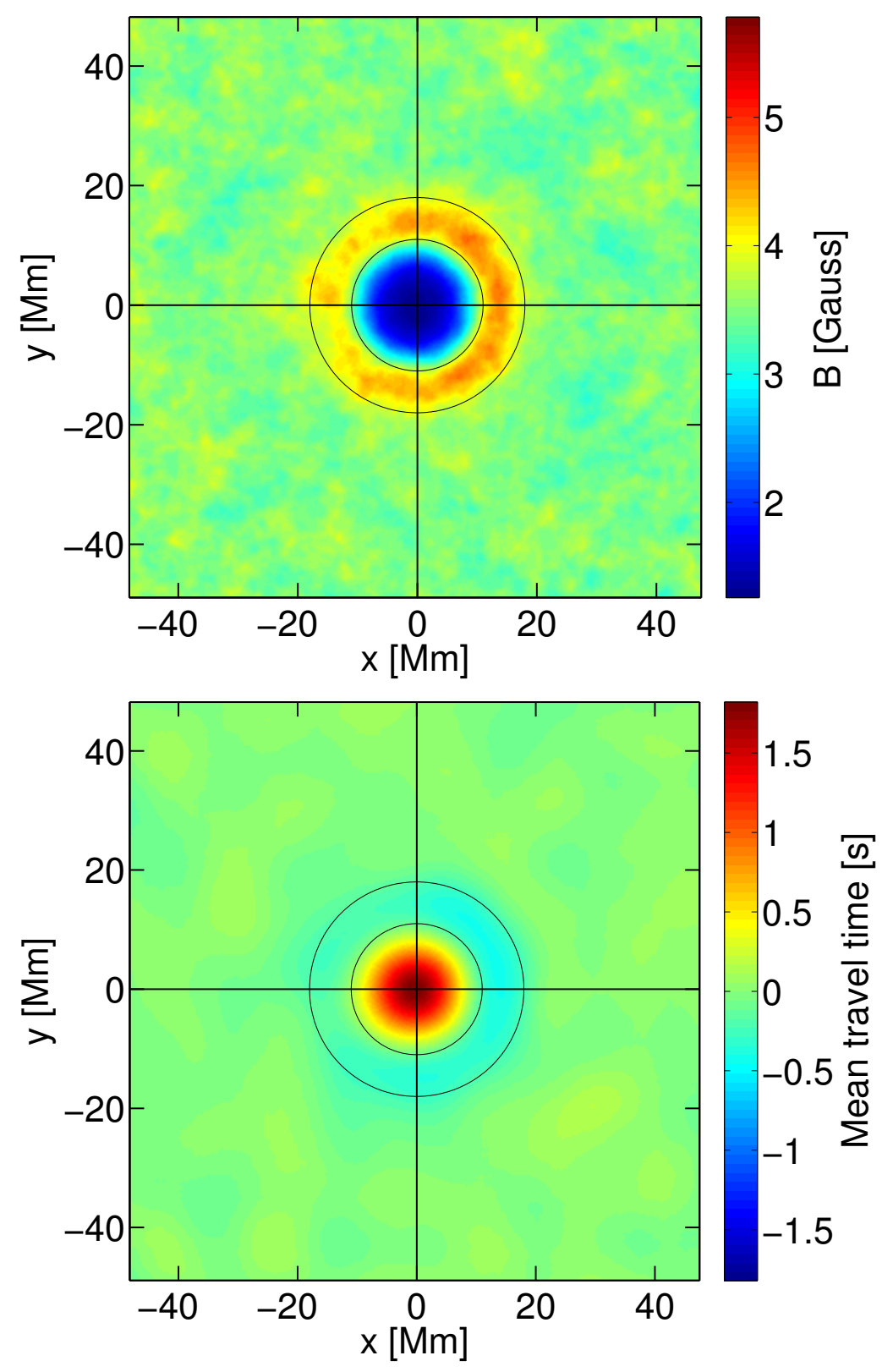

Figure 4.2: Line-of-sight magnetic field and mean travel times of the average supergranule near disk center, as measured from HMI. The black circles are centered on the origin and have radii of 11 and $18 \mathrm{Mm}$. Top: Absolute line-of-sight magnetic field $B$. The absolute value was taken after averaging $B_{\mathrm{LOS}}$ over $8 \mathrm{~h}$. The colorbar is symmetrized around the spatial average (3.52 Gauss). Bottom: Mean travel times for $\mathrm{f}$ modes (surface gravity waves). These travel times are the mean of outward and inward travel times measured between a central point and a surrounding annulus of radius $10 \mathrm{Mm}$ and are sensitive to the magnetic field. The colorbar is symmetrized around zero (which is the mean value by construction).

A similar anisotropy is observed in the mean travel times (right panel) with a larger amplitude. In the center of the supergranule, the mean travel times are longer than in 

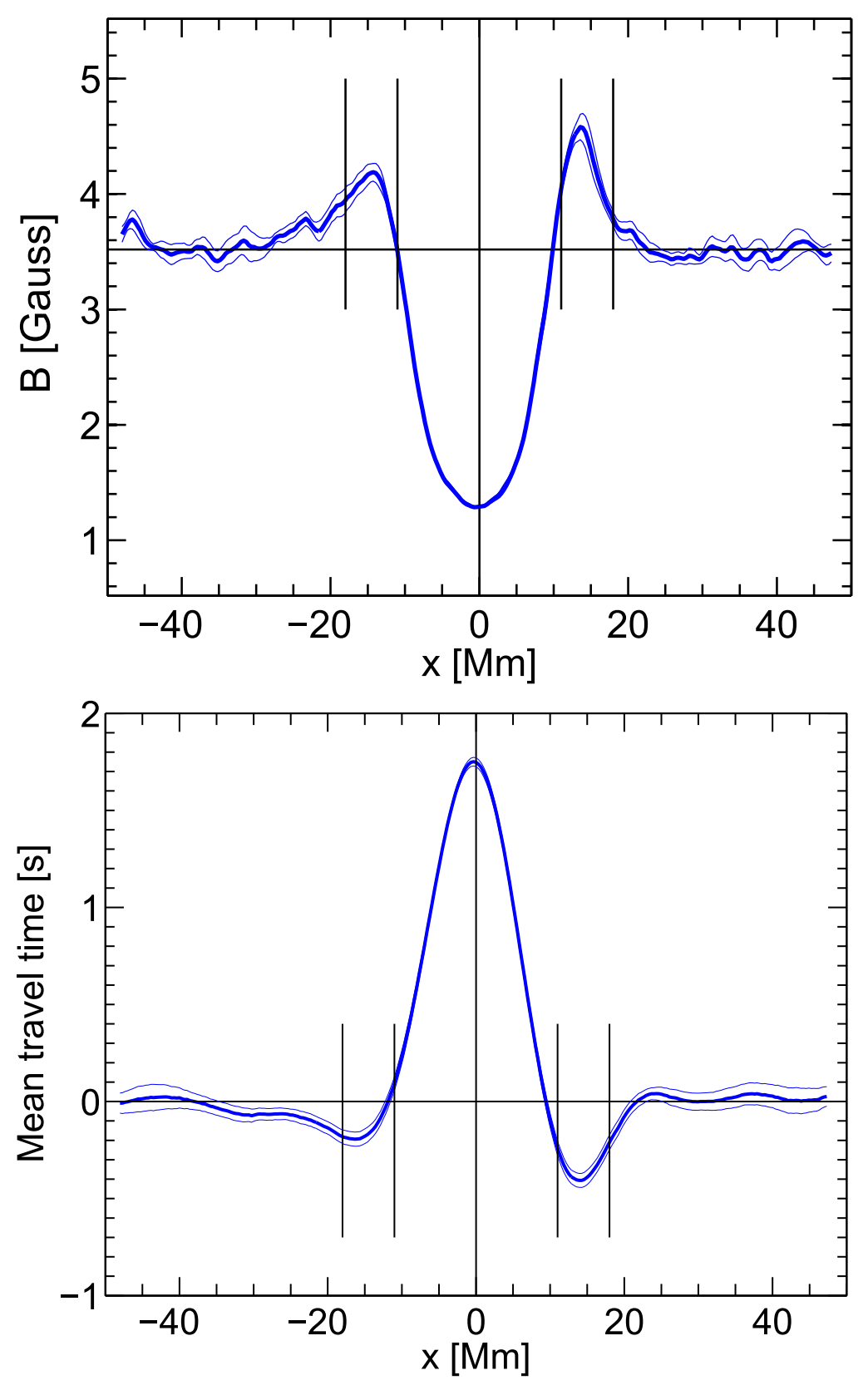

Figure 4.3: Cuts along $x$ axis (averaged over a strip $|y|<2.5 \mathrm{Mm}$ ) of the panels in Fig. 4.2. The thin blue lines give the $1 \sigma$ bounds, and the vertical lines indicate the position of the ring (network) shown in the previous plots. Top: Line-of-sight magnetic field, $B$. The horizontal black line shows the spatial average (3.52 Gauss). Bottom: Mean travel times for $f$ modes.

the inflow region where the magnetic field is stronger. In comparison to the divergence map in Fig. 4.1, the central peak of the mean travel time is slightly shifted to the east. The peak amplitude of the mean travel times (presumably caused by magnetic field, e.g., Duvall et al. 2006) is smaller by a factor of 25 compared to the peak in the travel-time differences (mostly caused by radial outflows with a peak velocity of about $300 \mathrm{~m} \mathrm{~s}^{-1}$, Langfellner et al. 2015). 
Figure 4.3 shows plots along the $x$-axis through the maps from Fig. 4.2, after averaging over a band $|y|<2.5 \mathrm{Mm}$. The dip of the magnetic field in the center of the supergranule is fairly flat compared to a Gaussian profile and has a full width at half maximum (FWHM) of about $16 \mathrm{Mm}$. On the west side of the surrounding ring, the field is about 0.3 to 0.4 Gauss stronger than on the east side. (This difference corresponds to more than $3 \sigma$.) The maximum field is attained at $x= \pm 13 \mathrm{Mm}$. For the mean travel times, the FWHM of the central peak is about $12 \mathrm{Mm}$ and more Gaussian in shape. The zero-crossing positions are found at $x=9$ and $x=-12 \mathrm{Mm}$. Furthermore, the minima are at different distances (13 and $15 \mathrm{Mm}$ ). The mean travel time on the west side has about twice the magnitude than on the east side.

Our findings can be compared to Duvall \& Birch (2010), who measured the absolute line-of-sight magnetic field of the average supergranule using data from the Michelson Doppler Imager (MDI) (Scherrer et al. 1995) onboard the Solar and Heliospheric Observatory (SOHO), albeit for the azimuthally averaged $B$. Their profile of $B$ as a function of the distance from the center of the average supergranule agrees with our measurements, but there are differences in the details of the curves. Duvall \& Birch measured a small bump in the central dip (perhaps not significant) and a maximum located at a distance of about $18 \mathrm{Mm}$ to the supergranule center, thus farther away than in our measurements $(13 \mathrm{Mm})$. This is probably due to a different selection of supergranulation sizes. Their magnetic field is about 2 Gauss in the dip and has a maximum of 5.5 Gauss. Their average field is roughly 4 Gauss, close to our value of 3.52 Gauss. Duvall \& Birch averaged only over $4 \mathrm{~h}$ per map, though, which could explain their stronger average field.

\subsubsection{Measuring the anisotropy of the network field}

To analyze the anisotropy of the network field in more detail, we study $B$ in the network as a function of azimuthal angle $\psi$, where $\psi=0^{\circ}$ points west and $\psi=90^{\circ}$ points north. This is accomplished by averaging $B$ between the circles with radii 11 and $18 \mathrm{Mm}$ shown in Fig. 4.2 over azimuthal segments of width $10^{\circ}$. We call the result $B_{\text {network}}$. We apply the same procedure to the mean travel times.

The azimuthal dependence of $B_{\text {network }}$ is shown in Fig. 4.4, As already seen in Fig. 4.2, $B_{\text {network }}$ is significantly larger near $\psi=0^{\circ}$ (in the west direction) than near $\psi=180^{\circ}$ (east direction). We find that the azimuthal dependence of $B_{\text {network }}$ can be described by

$$
B_{\text {network }} \approx 3.52+0.63(1+0.23 \cos \psi) \text { Gauss. }
$$

This means that $B_{\text {network }}$ varies from 4.0 Gauss in the east to 4.3 Gauss in the west. For the mean travel times (right panel of Fig. 4.4), the situation is similar, but the variations have opposite signs:

$$
\delta \tau_{\text {network }} \approx-0.25(1+0.40 \cos \psi) \mathrm{s} .
$$

One may ask whether the anisotropy in the mean travel times is caused by instrumental errors. We believe that this is unlikely. First, the HMI instrument shows no astigmatism for any practical purposes (in particular, the power of solar oscillations is isotropic at disk center). Second, we find that the anisotropy in the travel times around the average supergranule is the same for patches along the equator but centered at longitudes $\pm 20^{\circ}$, $\pm 40^{\circ}$, and $\pm 60^{\circ}$ away from the central meridian, averaged over east and west. 

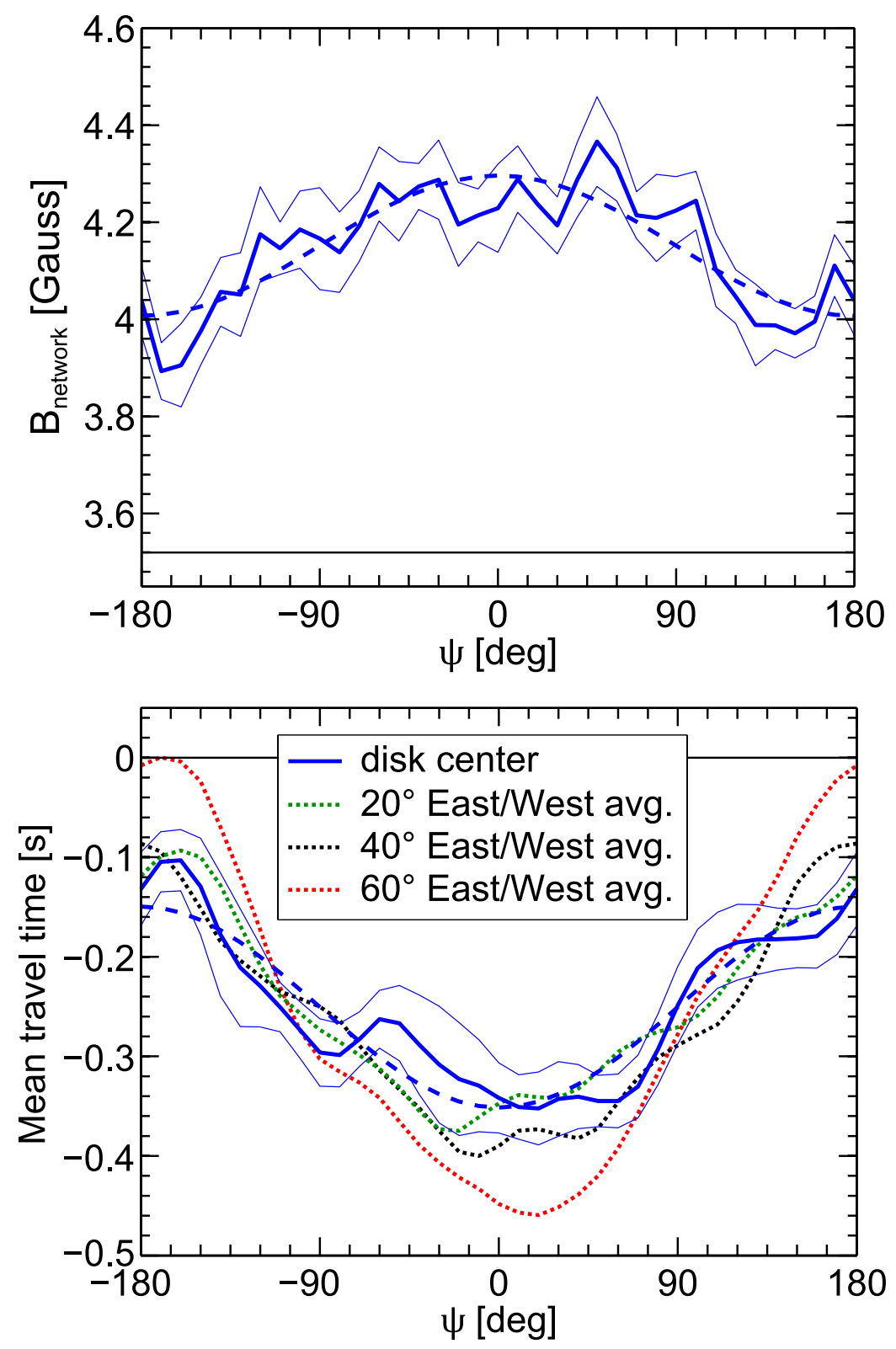

Figure 4.4: Top: Magnetic field $B_{\text {network }}$ around the average supergranule near disk center as a function of azimuthal angle $\psi$. To obtain $B_{\text {network }}$, the magnetic field $B$ was averaged over segments (width $10^{\circ}$ in $\psi$ ) of a concentric ring around the center of the average supergranule. The inner and outer limits of the ring are 11 and $18 \mathrm{Mm}$ (circles in Fig. 4.2). The thin blue lines give the $1 \sigma$ bounds. The dashed line is a least-squares fit to a cosine of the form specified by Eq. (4.1). The maximum of the cosine is fixed at $\psi=0^{\circ}$ (west direction). For comparison, the horizontal black line shows the background field $\bar{B}=$ 3.52 Gauss. Bottom: As above, but for f-mode mean travel times. In addition to the result at disk center, the mean travel times at other positions along the equator are shown (using patches centered at longitudes $\pm 20^{\circ}, \pm 40^{\circ}$, and $\pm 60^{\circ}$ away from the central meridian, averaged over east and west). The dashed line represents the cosine function given by Eq. (4.2). By construction, the mean travel time in the background is zero. 
Because it is known that f-mode travel times are reduced in magnetic regions (e.g., Gizon 2006, Duvall et al. 2006), the two results for $B_{\text {network }}$ and $\delta \tau_{\text {network }}$ appear to be consistent. We note that the relative variations of the travel times $(0.40)$ are larger than the relative variations of the magnetic field (0.23). Furthermore, by considering all the points in the average-supergranule maps, we find a one-to-one relationship between $\delta \tau$ and $B$. By choosing the center of the average supergranule as the reference point with $B_{\min }=1.3$ Gauss and $\delta \tau_{\max }=1.8 \mathrm{~s}$, this relationship can be described by

$$
\delta \tau \sim \delta \tau_{\max }-\left(1.2 \mathrm{~s} \mathrm{Gauss}^{-1 / 2}\right)\left(B-B_{\min }\right)^{1 / 2} .
$$

This suggests that the magnetic field anisotropy leaves its signature in the travel-time perturbations. However, it is not excluded that an anisotropy in the flows could also affect the travel times.

\subsection{Conclusion}

Using data from SDO/HMI, we have measured the line-of-sight component of the magnetic field of the average supergranule near disk center (ensemble average over about 3000 supergranules). We detected an unexpected anisotropy of the network field that surrounds the average supergranule. The magnetic field is stronger west of the average supergranule. Similarly, the mean travel time is decreased west of the average supergranule.

The measured anisotropy of the magnetic field is but one result in a series of puzzling observations associated with supergranulation. Other puzzling observations include, for example, the finding that the supergranulation pattern rotates faster around the Sun than magnetic features (e.g., Snodgrass \& Ulrich 1990, Meunier \& Roudier 2007) and the discovery of wavelike properties of supergranulation (Gizon et al. 2003, Schou 2003). How these phenomena are related to the discovery in this paper is unclear.

\section{Acknowledgements}

JL, LG, and ACB designed the research. JL performed research, analyzed data, and wrote the paper. We thank T. Duvall and J. Schou for useful discussions. JL and LG acknowledge research funding by Deutsche Forschungsgemeinschaft (DFG) under grant SFB 963/1 "Astrophysical flow instabilities and turbulence" (Project A1). The HMI data used are courtesy of NASA/SDO and the HMI science team. The data were processed at the German Data Center for SDO (GDC-SDO), funded by the German Aerospace Center (DLR). Support is acknowledged from the SPACEINN and SOLARNET projects of the European Union. We are grateful to R. Burston and H. Schunker for providing help with the data processing, especially the tracking and mapping. We used the workflow management system Pegasus funded by The National Science Foundation under OCI SI2-SSI program grant \#1148515 and the OCI SDCI program grant \#0722019. 


\section{Discussion}

In this thesis, we developed new measurement techniques to probe vortical flows in the solar interior on supergranulation scale and larger. We obtained vorticity maps of the average supergranule, which reveal the effects of rotation on convective flows. At $40^{\circ}$ latitude, the amplitude of the tangential flow component is roughly $10 \mathrm{~m} \mathrm{~s}^{-1}$, corresponding to $5 \%$ of the outflow amplitude. The latitudinal dependence of the vorticity amplitude (proportional to $\Omega(\lambda) \sin \lambda$ ) points to the Coriolis force as the physical explanation.

In the following, we expand the discussion of the flow velocity and vorticity profiles in Sect. 3.6 and compare these measurements to some simple models.

\subsection{Toy models for vortical flows}

We start by constructing a simplistic toy model for the tangential flow component $v_{t}$ that only considers advection and the Coriolis force. We choose a cylindrical coordinate system with the origin placed on the center of the supergranular outflow and refer to $r$ as the horizontal distance of a point to the origin (cf. Fig. 3.1k). Furthermore, we neglect the vertical direction, and assume that the average supergranular outflow profile $v_{r}(r)$ is stationary and isotropic in the horizontal plane, and dominates over the tangential flow component $\left(v_{r} \gg v_{t}\right)$. The momentum equation for the tangential flow component $v_{t}$ is then given by a balance of advection and Coriolis acceleration, $a_{c}=-2 v_{r} \Omega(\lambda) \sin \lambda$ :

$$
\frac{D}{D t} v_{t}=a_{c}
$$

where $D / D t$ is the material derivative. This corresponds to a test particle being advected by the supergranular outflow and being accelerated by the Coriolis force. Assuming the boundary condition $v_{t}(r=0)=0$, the solution to this equation is

$$
v_{t}(r)=-r \Omega(\lambda) \sin \lambda,
$$

and thus is independent of $v_{r}$. This linear profile is plotted with the measured profiles of $v_{t}$ and $v_{r}$ from LCT in Fig. B.5 in the Appendix. Surprisingly, the slope from this extremely simple toy model matches the measured slope closely near the center of the average outflow, up to $r \approx 5 \mathrm{Mm}$, for all analyzed latitudes (from $-60^{\circ}$ to $60^{\circ}$ ).

Note that the linear $v_{t}$ profile implies that the vorticity is constant and given by

$$
\omega_{z}=-2 \Omega(\lambda) \sin \lambda
$$

in the toy model. Thus the vorticity is only determined by the local solar rotation rate. For $\lambda=40^{\circ}$, this relation gives $\omega_{z} \approx 3 \times 10^{-6} \mathrm{~s}^{-1}$. This is in line with the observed vorticity plateau (Fig. 3.12a). 
For the average inflow, the same model gives a diverging $v_{t}$ as $r \rightarrow 0$ (if the fluid has some $v_{t}(R)$ at some distance $R$ that does not exactly match Eq. 5.2), which is not observed ( $v_{t}$ approaches zero). However, this mismatch is not surprising because a strong gradient in $v_{t}$ would be moderated by the turbulent viscosity, a quantity that has been neglected in the toy model. As we discussed in Sect. 3.6, a model of a vortex that decays due to turbulent viscosity is indeed able to describe the $v_{t}$ profile of the average inflow region quite well.

While the toy model is able to reproduce the measured tangential velocity and vertical vorticity in the innermost part of the average supergranular outflow, and also might provide an idea for why the $v_{t}$ profiles look different in outflows and inflows, it is also worth looking at models that include additional physical ingredients.

A more sophisticated model of convective flows that includes viscosity was provided by Veronis (1959) and used by Duvall \& Gizon (2000). In this model, convection cells are approximated as hexagonal cells, with the outflow center in the middle of the hexagon and the inflow centers at the vertices. This pattern is assumed to be periodic. As outlined in Sect. 3.6, the Veronis model provides a close relationship between $v_{t}$ and $v_{r}$. Choosing the direction that contains both the outflow and inflow centers, the profiles for $v_{r}$ and $v_{t}$ are given by

$$
\begin{aligned}
& v_{r}(r)=v_{0} \sin \left(\frac{\pi}{L} r\right) \cos \left(\frac{\pi}{3 L} r\right), \\
& v_{t}(r)=v_{0 t} \sin \left(\frac{\pi}{L} r\right) \cos \left(\frac{\pi}{3 L} r\right) \quad \text { with } \quad v_{0 t}=-v_{0} \frac{2 l^{2}}{\pi^{2}\left(4+l^{2} / d^{2}\right)} \frac{\Omega(\lambda) \sin \lambda}{\eta},
\end{aligned}
$$

with the edge length $L$ of the hexagon and some velocity scale $v_{0}$ (roughly the maximum outflow speed). The quantity $l=\sqrt{3} L$ is the spatial periodicity length of the hexagonal grid, $d$ is the depth of the supergranule, and $\eta$ is the kinematic viscosity. Fits of these functions (with the free parameters $v_{0}, v_{0 t}$, and $L$ ) to the measured $v_{r}$ and $v_{t}$ profiles at $40^{\circ}$ latitude (cf. Fig. 3.13) are shown in Fig. 5.1. We arbitrarily restricted the fit range to a maximum distance $r=15 \mathrm{Mm}$. Compared to the toy model shown before, the Veronis model is able to follow the measured profiles of $v_{r}$ and $v_{t}$ more closely. However, the close fit of the model to the measured profiles in the outflows is only possible because individual values for $L$ for $v_{r}$ and $v_{t}$ were allowed. The different extrema positions of $v_{r}$ and $v_{t}$ cannot be explained with the Veronis model. For the inflows, the fit of the model follows the measured $v_{t}$ profile only roughly.

One reason for this could be that the underlying symmetries that are assumed in the Veronis model are not fulfilled by the individual supergranular outflows and inflows that were selected to construct the average supergranular outflows and inflows. In the future, it might be instructive to specifically search for (the presumably small subset of) supergranules that are surrounded by six other supergranules and compare again to the Veronis model.

Another model, which considers the balance between an inflow and outward diffusion due to a kinematic viscosity (but does not contain rotation), was provided by Simon \& Weiss (1997). The tangential velocity component in this model is given by

$$
v_{t}(r)=\frac{\Gamma}{2 \pi r}\left[1-\exp \left(-\frac{r^{2}}{r_{0}^{2}}\right)\right],
$$



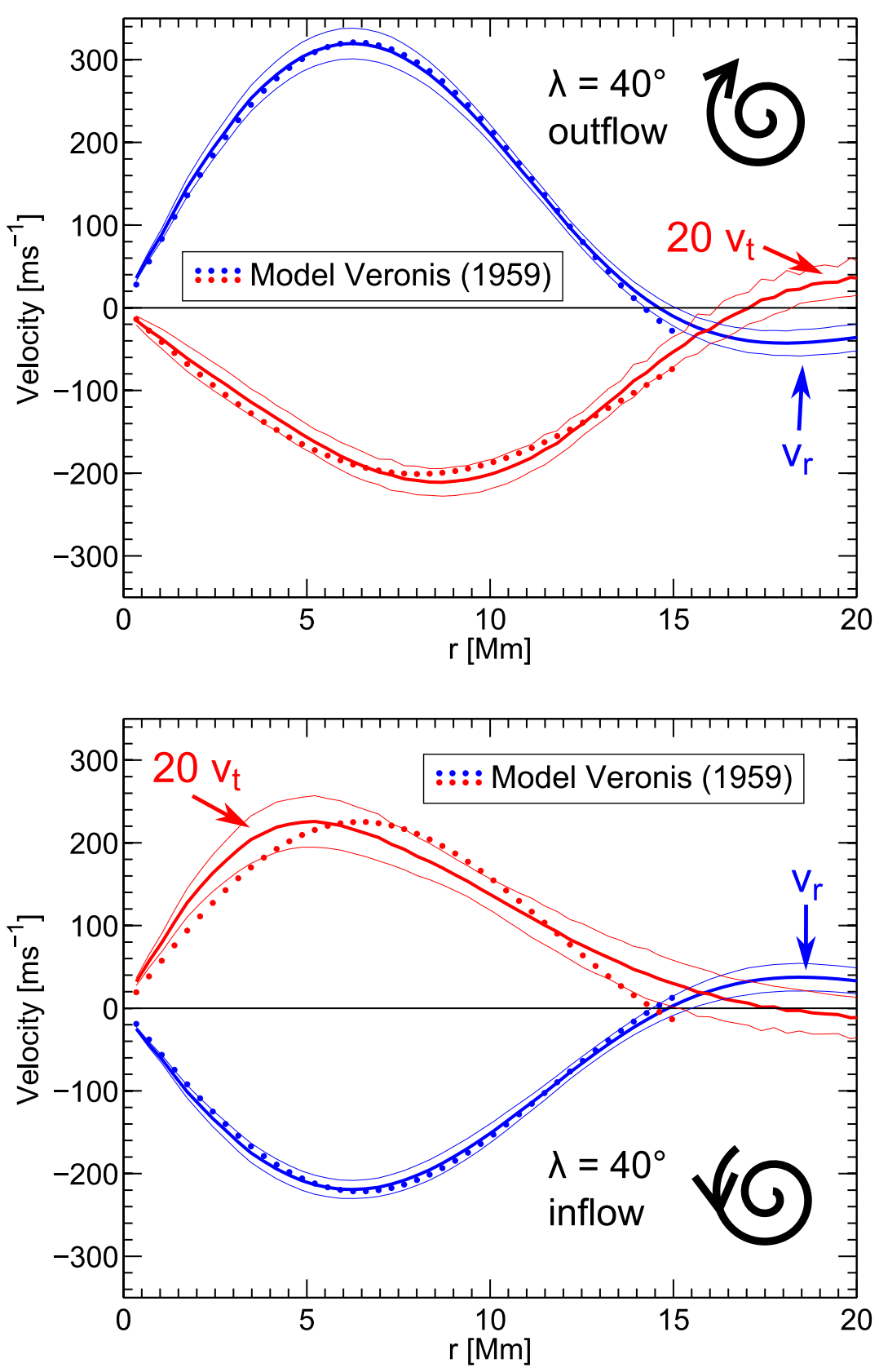

Figure 5.1: Azimuthally averaged horizontal velocities around the average supergranule outflow and inflow centers at solar latitude $40^{\circ}$ (cf. Fig. 3.13), from measurements and a convection model. Top: Horizontal velocities $v_{r}$ and $v_{t}$ around the average supergranule outflow center. The measurements (thick lines) were obtained from LCT of granules in HMI intensity images. The thin lines mark an estimate of the variability of the data as measured by dividing the 336 datasets into eight parts. For $v_{r}$, the $10 \sigma$ level is shown, for $v_{t}$ the $3 \sigma$ level. The dotted lines show a least-squares fit of the model by Veronis (1959) (Eqs. 5.4 and 5.5) to the measurements. Bottom: As above, but around the average supergranule inflow center. 

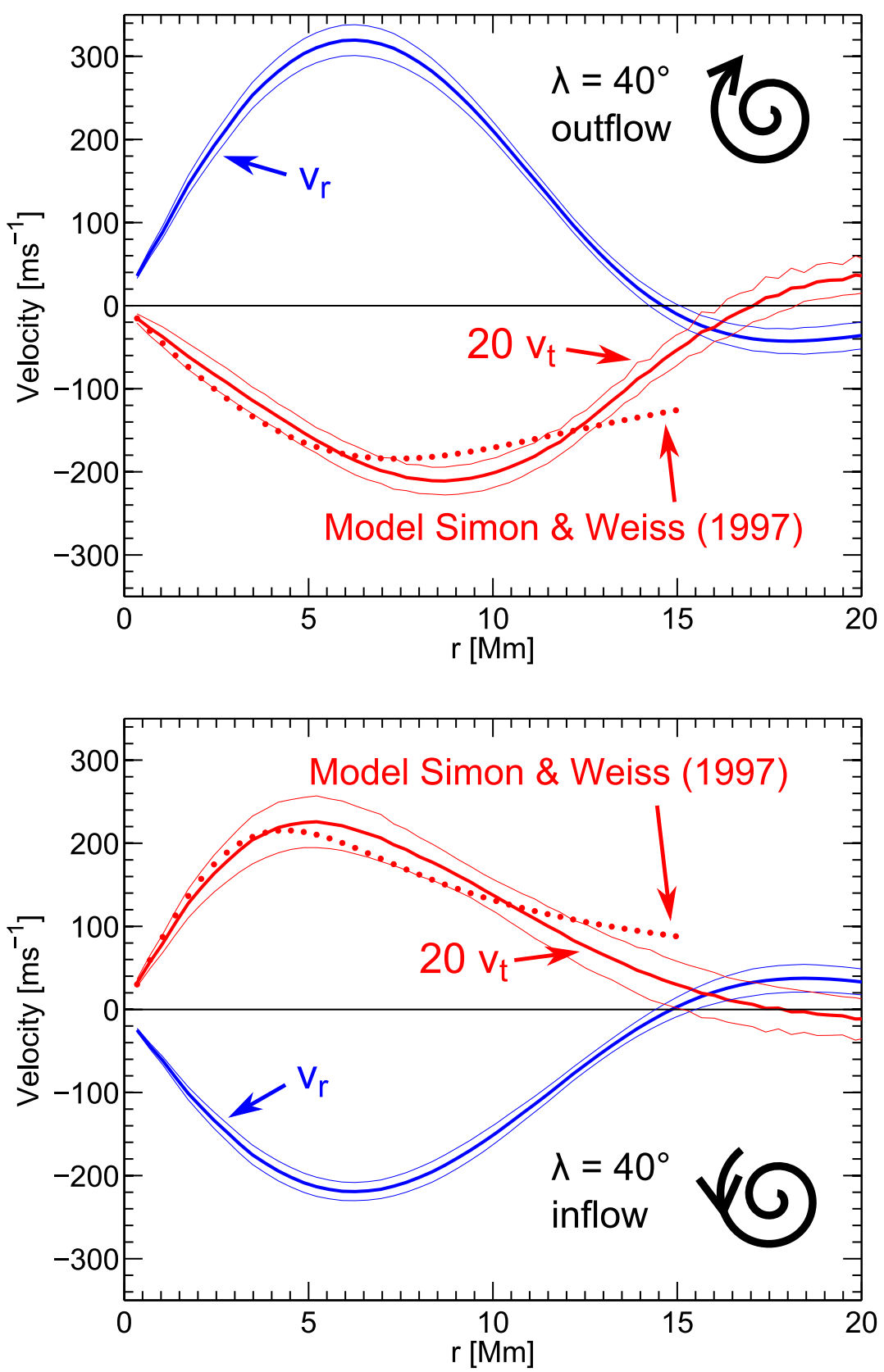

Figure 5.2: As Fig. 5.1, but with a fit of the model by Simon \& Weiss (1997) (Eq. 5.6) to the tangential velocity component $v_{t}$. 
with some circulation $\Gamma$ and vortex radius $r_{0}$. Figure 5.2 shows fits to the outflow and inflow at $40^{\circ}$ latitude (with $\Gamma$ and $r_{0}$ being free parameters). For the average inflow, the fit is a good approximation to the inner part, but it fails further outward (beyond $r=10 \mathrm{Mm}$ ). Presumably, the reason for this is that the model does not take into account surrounding outflows. Thus the function in Eq. (5.6) does not switch sign at any distance but instead slowly approaches zero, in contrast to the measured $v_{t}$ profile. For the sake of completeness, we also show the fit for the outflow. Obviously, the fit is worse than for the inflow. Since the model was designed for inflows, this is not surprising though.

To conclude, existing models of vortical flows in solar supergranulation are only partly consistent with the measurements obtained in this thesis. While they can provide useful hints for the physical ingredients involved, further theoretical work is required to explain the measured flow profiles.

\subsection{Outlook}

This work has revealed some hitherto unknown facets of supergranulation, but its nature remains elusive. Further observational studies are needed in the future to provide a more comprehensive picture of solar supergranulation and larger-scale flows. Some suggestions for future work include:

- Dynamics of giant cells. It was shown that flows on larger scales than supergranulation can be probed by vorticity measurement techniques. Using bigger maps and more data, the impact of solar rotation on giant cells (Hathaway et al. 2013) could be spatially resolved and compared to simulations (e.g., Miesch et al.|2008).

- Vertical structure of $\omega_{z}$. The vorticity-sensitive travel times $\tau^{\mathrm{ac}}$ are only an intermediate data product. Inversions of the travel-time maps can yield 3D maps of $\omega_{z}$. This would tell us at which depth the vorticity of the average supergranule switches sign and thus provide an estimate of the supergranulation depth. Such a study could benefit from an inversion technique that has recently been adapted to helioseismology (the so-called Pinsker estimator, see Pinsker 1980, Fournier et al. 2015).

- Connection to large-scale dynamics. As was measured in Paper I, there is a net kinetic helicity $\langle\mathbf{v} \cdot \boldsymbol{\omega}\rangle$ in supergranular flows. Thus supergranulation does not only disperse the magnetic field, but also twists the field. This is directly related to the $\alpha$ effect in mean field theory (Krause \& Rädler 1980, Rüdiger et al. 1999). The same theory also predicts that the Sun's rotation introduces correlations between the horizontal flow velocity components $\left\langle v_{x} v_{y}\right\rangle$, which is thought to maintain the differential rotation (e.g., Kitchatinov \& Rüdiger 1993). A measurement of the horizontal Reynolds stress at supergranulation scales versus latitude could thus be used to put constraints on mean field theory.

- Evolution of supergranules. With longer tracking periods, identified supergranules could be followed for several days. This would allow a study of the evolution of the flows and of the magnetic field of the average supergranule and could provide hints regarding the origin of supergranules. 
Apart from observational studies, realistic numerical simulations are needed to understand supergranules and larger-scale flows from a theoretical point of view. Whereas a model of convection that includes supergranulation appears to be out of reach for the foreseeable future, the ongoing developments in computational power stir up hope that supergranulation and giant cells might be successfully reproduced in simulations in the coming years. 


\section{Bibliography}

Aerts, C., Christensen-Dalsgand, J. \& Kurtz, D. W. (2010) Asteroseismology. 1st edn., Springer.

Appourchaux, T., Belkacem, K., Broomhall, A.-M., Chaplin, W. J., Gough, D. O., Houdek, G., Provost, J., Baudin, F. et al. (2010) The quest for the solar g modes. AEA Rev., 18, 197-277.

Attie, R., Innes, D. E. \& Potts, H. E. (2009) Evidence of photospheric vortex flows at supergranular junctions observed by FG/SOT (Hinode). AEA A, 493, L13-L16.

Baвсоск, H. W. (1961) The Topology of the Sun's Magnetic Field and the 22-YEAR Cycle. ApJ, 133, 572.

Barekat, A., Schou, J. \& Gizon, L. (2014) The radial gradient of the near-surface shear layer of the Sun. AEA, 570, L12.

Birch, A. C. \& Gizon, L. (2007) Linear sensitivity of helioseismic travel times to local flows. Astronomische Nachrichten, 328, 228.

BöHM-Vitense, E. (1958) Über die Wasserstoffkonvektionszone in Sternen verschiedener Effektivtemperaturen und Leuchtkräfte. Mit 5 Textabbildungen. ZAp, 46, 108.

Brandt, P. N., Scharmer, G. B., Ferguson, S., Shine, R. A. \& Tarbell, T. D. (1988) Vortex flow in the solar photosphere. Nature, 335, 238-240.

Braun, D. C., Duvall, JR., T. L. \& Labonte, B. J. (1987) Acoustic absorption by sunspots. ApJ, 319, L27-L31.

Cameron, R. \& Schüssler, M. (2015) The crucial role of surface magnetic fields for the solar dynamo. Science, 347, 1333-1335.

Charbonneau, P. (2010) Dynamo Models of the Solar Cycle. Living Reviews in Solar Physics, 7, 3.

Chou, D.-Y., Sun, M.-T., Huang, T.-Y., LaI, S.-P., Chi, P.-J., Ou, K.-T., Wang, C.-C., Lu, J.-Y. et AL. (1995) Taiwan Oscillation Network. Sol. Phys., 160, 237-243.

Christensen-Dalsgaard, J. (2002) Helioseismology. Rev. Mod. Phys., 74, 1073-1129.

Christensen-Dalsgaard, J., Duvall, Jr., T. L., Gough, D. O., Harvey, J. W. \& Rhodes, JR., E. J. (1985) Speed of sound in the solar interior. Nature, 315, 378-382. 
Christensen-Dalsgaard, J., Gough, D. O. \& Thompson, M. J. (1991) The depth of the solar convection zone. ApJ, 378, 413-437.

Couvidat, S., Rajaguru, S. P., Wachter, R., Sankarasubramanian, K., Schou, J. \& ScherRER, P. H. (2012a) Line-of-Sight Observables Algorithms for the Helioseismic and Magnetic Imager (HMI) Instrument Tested with Interferometric Bidimensional Spectrometer (IBIS) Observations. Sol. Phys., 278, 217-240.

Couvidat, S., Schou, J., Shine, R. A., Bush, R. I., Miles, J. W., Scherrer, P. H. \& RairDEN, R. L. (2012b) Wavelength Dependence of the Helioseismic and Magnetic Imager (HMI) Instrument onboard the Solar Dynamics Observatory (SDO). Sol. Phys., 275, $285-325$.

Crouch, A. D., Cally, P. S., Charbonneau, P., Braun, D. C. \& Desjardins, M. (2005) Genetic magnetohelioseismology with Hankel analysis data. MNRAS, 363, 1188-1204.

Crouch, A. D., Charbonneau, P. \& Thibault, K. (2007) Supergranulation as an Emergent Length Scale. ApJ, 662, 715-729.

De Rosa, M., Duvall, Jr., T. L. \& Toomre, J. (2000) Near-Surface Flow Fields Deduced Using Correlation Tracking and Time-Distance Analyses. Sol. Phys., 192, 351-361.

De Rosa, M. L. \& Toomre, J. (1998) Correlation Tracking of Mesogranules from SOIMDI Doppler Images to Reveal Supergranular Flow Fields. In Structure and Dynamics of the Interior of the Sun and Sun-like Stars, edited by S. Korzennik, vol. 418 of ESA Special Publication.

De Rosa, M. L. \& Toomre, J. (2004) Evolution of Solar Supergranulation. ApJ, 616, $1242-1260$.

Domingo, V., Fleck, B. \& Poland, A. I. (1995) The SOHO Mission: an Overview. Sol. Phys., 162, 1-37.

Duvall, T. L., D’Silva, S., Jefferies, S. M., Harvey, J. W. \& Schou, J. (1996) Downflows under sunspots detected by helioseismic tomography. Nature, 379, 235-237.

Duvall, T. L. \& Hanasoge, S. M. (2013) Subsurface Supergranular Vertical Flows as Measured Using Large Distance Separations in Time-Distance Helioseismology. Sol. Phys., 287, 71-83.

Duvall, T. L., Hanasoge, S. M. \& Chakraborty, S. (2014) Additional Evidence Supporting a Model of Shallow, High-Speed Supergranulation. Sol. Phys., 289, 3421-3433.

Duvall, JR., T. L. (1980) The equatorial rotation rate of the supergranulation cells. Sol. Phys., 66, 213-221.

Duvall, JR., T. L. \& Birch, A. C. (2010) The Vertical Component of the Supergranular Motion. ApJ, 725, L47-L51.

Duvall, JR., T. L., Birch, A. C. \& Gizon, L. (2006) Direct Measurement of Travel-Time Kernels for Helioseismology. ApJ, 646, 553-559. 
Duvall, Jr., T. L., Dziembowski, W. A., Goode, P. R., Gough, D. O., Harvey, J. W. \& LeIBACHER, J. W. (1984) Internal rotation of the sun. Nature, 310, 22-25.

Duvall, JR., T. L. \& Gizon, L. (2000) Time-Distance Helioseismology with f Modes as a Method for Measurement of Near-Surface Flows. Sol. Phys., 192, 177-191.

Duvall, JR., T. L., Jefferies, S. M., Harvey, J. W., Osaki, Y. \& Pomerantz, M. A. (1993a) Asymmetries of solar oscillation line profiles. ApJ, 410, 829-836.

Duvall, Jr., T. L., Jefferies, S. M., Harvey, J. W. \& Pomerantz, M. A. (1993b) Timedistance helioseismology. Nature, 362, 430-432.

Duvall, Jr., T. L., Kosovichev, A. G., Scherrer, P. H., Bogart, R. S., Bush, R. I., De Forest, C., Hoeksema, J. T., Schou, J. et Al. (1997) Time-Distance Helioseismology with the MDI Instrument: Initial Results. Sol. Phys., 170, 63-73.

Fisher, G. H. \& Welsch, B. T. (2008) FLCT: A Fast, Efficient Method for Performing Local Correlation Tracking. In Subsurface and Atmospheric Influences on Solar Activity, edited by R. Howe, R. W. Komm, K. S. Balasubramaniam \& G. J. D. Petrie, vol. 383 of Astronomical Society of the Pacific Conference Series.

Fournier, D., Gizon, L., Hohage, T. \& Birch, A. C. (2014) Generalization of the noise model for time-distance helioseismology. AEFA, 567, A137.

Fournier, D., Gizon, L., Hohage, T. \& Holzke, M. (2015) Pinsker estimators for local helioseismology. ArXiv e-prints.

Galloway, D. J., Proctor, M. R. E. \& Weiss, N. O. (1977) Formation of intense magnetic fields near the surface of the sun. Nature, 266, 686-689.

Gizon, L. (2006) Probing Convection and Solar Activity with Local Helioseismology. In SOHO-17. 10 Years of SOHO and Beyond, vol. 617 of ESA Special Publication.

Gizon, L. \& Birch, A. C. (2004) Time-Distance Helioseismology: Noise Estimation. ApJ, 614, 472-489.

Gizon, L. \& Birch, A. C. (2005) Local Helioseismology. Living Reviews in Solar Physics, 2,6 .

Gizon, L., Birch, A. C. \& Spruit, H. C. (2010) Local Helioseismology: ThreeDimensional Imaging of the Solar Interior. ARAE $A$, 48, 289-338.

Gizon, L., Duvall, T. L. \& Schou, J. (2003) Wave-like properties of solar supergranulation. Nature, 421, 43-44.

Gizon, L., Duvall, JR., T. L. \& Larsen, R. M. (2000) Seismic Tomography of the Near Solar Surface. Journal of Astrophysics and Astronomy, 21, 339.

Gizon, L., Duvall, JR., T. L. \& Larsen, R. M. (2001) Probing Surface Flows and Magnetic Activity with Time-Distance Helioseismology. In Recent Insights into the Physics of the Sun and Heliosphere: Highlights from SOHO and Other Space Missions, edited by P. Brekke, B. Fleck \& J. B. Gurman, vol. 203 of IAU Symposium. 
Goldbaum, N., Rast, M. P., Ermolli, I., Sands, J. S. \& Berrilli, F. (2009) The Intensity Profile of the Solar Supergranulation. ApJ, 707, 67-73.

Goldreich, P. \& Keeley, D. A. (1977) Solar seismology. II - The stochastic excitation of the solar p-modes by turbulent convection. ApJ, 212, 243-251.

González Hernández, I., Hill, F. \& Lindsey, C. (2007) Calibration of Seismic Signatures of Active Regions on the Far Side of the Sun. ApJ, 669, 1382-1389.

Gough, D. (2013) What Have We Learned from Helioseismology, What Have We Really Learned, and What Do We Aspire to Learn? Sol. Phys., 287, 9-41.

Greer, B. J., Hindman, B. W., Featherstone, N. A. \& Toomre, J. (2015) Helioseismic Imaging of Fast Convective Flows throughout the Near-surface Shear Layer. ApJ, 803, L17.

Hanasoge, S., Gizon, L. \& Sreenivasan, K. R. (2015) Seismic Sounding of Convection in the Sun. ArXiv e-prints.

Hanasoge, S. M., Duvall, T. L. \& Sreenivasan, K. R. (2012) Anomalously weak solar convection. Proceedings of the National Academy of Science, 109, 11928-11932.

Hart, A. B. (1954) Motions in the Sun at the photospheric level. IV. The equatorial rotation and possible velocity fields in the photosphere. MNRAS, 114, 17.

Hart, A. B. (1956) Motions in the Sun at the photospheric level. VI. Large-scale motions in the equatorial region. MNRAS, 116, 38.

Harvey, J. W., Hill, F., Hubbard, R. P., Kennedy, J. R., Leibacher, J. W., Pintar, J. A., Gilman, P. A., Noyes, R. W. ET AL. (1996) The Global Oscillation Network Group (GONG) Project. Science, 272, 1284-1286.

Hathaway, D. H., Beck, J. G., Bogart, R. S., Bachmann, K. T., Khatri, G., Petitto, J. M., Han, S. \& Raymond, J. (2000) The Photospheric Convection Spectrum. Sol. Phys., 193, 299-312.

Hathaway, D. H., Beck, J. G., Han, S. \& Raymond, J. (2002) Radial Flows in Supergranules. Sol. Phys., 205, 25-38.

Hathaway, D. H., Upton, L. \& Colegrove, O. (2013) Giant Convection Cells Found on the Sun. Science, 342, 1217-1219.

HILl, F. (1988) Rings and trumpets - Three-dimensional power spectra of solar oscillations. ApJ, 333, 996-1013.

Hindman, B. W., Haber, D. A. \& Toomre, J. (2009) Subsurface Circulations within Active Regions. ApJ, 698, 1749-1760.

Hirzberger, J., Gizon, L., Solanki, S. K. \& Duvall, T. L. (2008) Structure and Evolution of Supergranulation from Local Helioseismology. Sol. Phys., 251, 417-437. 
Hoeksema, J. T., Liu, Y., Hayashi, K., Sun, X., Schou, J., Couvidat, S., Norton, A., Bobra, M. ET AL. (2014) The Helioseismic and Magnetic Imager (HMI) Vector Magnetic Field Pipeline: Overview and Performance. Sol. Phys., 289, 3483-3530.

Hotta, H., Rempel, M. \& Yokoyama, T. (2014) High-resolution Calculations of the Solar Global Convection with the Reduced Speed of Sound Technique. I. The Structure of the Convection and the Magnetic Field without the Rotation. ApJ, 786, 24.

Houdek, G. \& Gough, D. O. (2011) On the seismic age and heavy-element abundance of the Sun. MNRAS, 418, 1217-1230.

Howe, R. (2009) Solar Interior Rotation and its Variation. Living Reviews in Solar Physics, 6, 1.

Jackiewicz, J., Gizon, L., Birch, A. C. \& Duvall, Jr., T. L. (2007) Time-Distance Helioseismology: Sensitivity of f-mode Travel Times to Flows. ApJ, 671, 1051-1064.

Jafarzadeh, S., Cameron, R. H., Solanki, S. K., Pietarila, A., Feller, A., Lagg, A. \& Gandorfer, A. (2014) Migration of Ca II H bright points in the internetwork. $A \mathcal{E} A$, 563, A101.

KipPEnHAhN, R. \& Weigert, A. (1990) Stellar Structure and Evolution. 1st edn., Springer.

Kitchatinov, L. L. \& RüDIGER, G. (1993) Lambda-effect and differential rotation in stellar convection zones. AEFA, 276, 96.

Komm, R., Howe, R., Hitl, F., Miesch, M., Haber, D. \& Hindman, B. (2007) Divergence and Vorticity of Solar Subsurface Flows Derived from Ring-Diagram Analysis of MDI and GONG Data. ApJ, 667, 571-584.

Korzennik, S. G., Rabello-Soares, M. C. \& Schou, J. (2004) On the Determination of Michelson Doppler Imager High-Degree Mode Frequencies. ApJ, 602, 481-516.

Korzennik, S. G., Rabello-Soares, M. C. \& Schou, J. (2012) Erratum: "On the Determination of Michelson Doppler Imager High-degree Mode Frequencies" (2004, ApJ, 602, 481). ApJ, 760, 156.

Kosugi, T., Matsuzaki, K., Sakao, T., Shimizu, T., Sone, Y., Tachikawa, S., Hashimoto, T., Minesugi, K. et AL. (2007) The Hinode (Solar-B) Mission: An Overview. Sol. Phys., 243, 3-17.

Krause, F. \& RäDler, K.-H. (1980) Mean-Field Magnetodynamics and Dynamo Theory. Pergamon Press.

Krijger, J. M. \& Roudier, T. (2003) Photospheric flows measured with TRACE II. Network formation. $A \mathcal{E} A, \mathbf{4 0 3}, 715-723$.

Langfellner, J., Gizon, L. \& Birch, A. C. (2014) Time-distance helioseismology: A new averaging scheme for measuring flow vorticity. $A \mathcal{E} A$, 570, A90. 
Langfellner, J., Gizon, L. \& Birch, A. C. (2015) Spatially resolved vertical vorticity in solar supergranulation using helioseismology and local correlation tracking. $A \mathcal{E} A, \mathbf{5 8 1}$, A67.

Leighton, R. B. (1964) Transport of Magnetic Fields on the Sun. ApJ, 140, 1547.

Leighton, R. B. (1969) A Magneto-Kinematic Model of the Solar Cycle. ApJ, 156, 1.

Leighton, R. B., Noyes, R. W. \& Simon, G. W. (1962) Velocity Fields in the Solar Atmosphere. I. Preliminary Report. ApJ, 135, 474.

Lindsey, C. \& Braun, D. C. (1990) Helioseismic imaging of sunspots at their antipodes. Sol. Phys., 126, 101-115.

Lisle, J. \& Toomre, J. (2004) Cause of Shrinking Sun Effect in Local Correlation Tracking and Impacts on the Mapping of Ssw Flows. In SOHO 14 Helio- and Asteroseismology: Towards a Golden Future, edited by D. Danesy, vol. 559 of ESA Special Publication.

Liu, Y., Hoeksema, J. T., Scherrer, P. H., Schou, J., Couvidat, S., Bush, R. I., Duvall, T. L., Hayashi, K. et al. (2012) Comparison of Line-of-Sight Magnetograms Taken by the Solar Dynamics Observatory/Helioseismic and Magnetic Imager and Solar and Heliospheric Observatory/Michelson Doppler Imager. Sol. Phys., 279, 295-316.

Löptien, B., Birch, A. C., Gizon, L. \& Schou, J. (2014a) Image compression in local helioseismology. AEFA, 571, A42.

Löptien, B., Birch, A. C., Gizon, L., Schou, J., Appourchaux, T., Blanco Rodríguez, J., Cally, P. S., Dominguez-Tagle, C. ET Al. (2014b) Helioseismology with Solar Orbiter. Space Sci. Rev., 1-33.

Lord, J. W., Cameron, R. H., Rast, M. P., Rempel, M. \& Roudier, T. (2014) The Role of Subsurface Flows in Solar Surface Convection: Modeling the Spectrum of Supergranular and Larger Scale Flows. ApJ, 793, 24.

Meunier, N. \& Roudier, T. (2007) The superrotation of solar supergranules. AEAA, 466, 691-696.

Meunier, N., Tkaczuk, R. \& Roudier, T. (2007) Intensity variations inside supergranules. $A \mathcal{E} A, \mathbf{4 6 3}, 745-753$.

Miesch, M. S., Brun, A. S., De Rosa, M. L. \& Toomre, J. (2008) Structure and Evolution of Giant Cells in Global Models of Solar Convection. ApJ, 673, 557-575.

Miesch, M. S., Featherstone, N. A., Rempel, M. \& Trampedach, R. (2012) On the Amplitude of Convective Velocities in the Deep Solar Interior. ApJ, 757, 128.

Munk, W. H., Worcester, P. \& Wunsch, C. (1995) Ocean acoustic tomography. 1st edn., Cambridge University Press.

Nordlund, A. (1985) Solar convection. Sol. Phys., 100, 209-235. 
Nordlund, Å., Stein, R. F. \& Asplund, M. (2009) Solar Surface Convection. Living Reviews in Solar Physics, 6, 2.

Norton, A. A., Graham, J. P., Ulrich, R. K., Schou, J., Tomczyk, S., Liu, Y., Lites, B. W., López Ariste, A. ET AL. (2006) Spectral Line Selection for HMI: A Comparison of Fe I $6173 \AA$ Å and Ni I 6768 A. Sol. Phys., 239, 69-91.

November, L. J. \& Simon, G. W. (1988) Precise proper-motion measurement of solar granulation. ApJ, 333, 427-442.

Orozco Suárez, D., Katsukawa, Y. \& Bellot Rubio, L. R. (2012) The Connection between Internetwork Magnetic Elements and Supergranular Flows. ApJ, 758, L38.

Parker, E. N. (1979) Cosmical magnetic fields: Their origin and their activity. Oxford.

Parnell, C. E. \& De Moortel, I. (2012) A contemporary view of coronal heating. Royal Society of London Philosophical Transactions Series A, 370, 3217-3240.

Pearson, K. (1901) On the lines and planes of closest fit to systems of points in space. Philosophical Magazine, 2, 559-572.

Pesnell, W. D., Thompson, B. J. \& Chamberlin, P. C. (2012) The Solar Dynamics Observatory (SDO). Sol. Phys., 275, 3-15.

Pinsker, M. S. (1980) Optimal Filtering of square-integrable signals in Gaussian white noise. Probl. Inf. Transm., 16, 52-68.

Pötzi, W. \& Brandt, P. N. (2007) Divergence and Vorticity at Solar Mesogranular Scales. Central European Astrophysical Bulletin, 31, 11.

Rast, M. P. (2003a) Supergranulation: new observation, possible explanation. In GONG+2002. Local and Global Helioseismology: the Present and Future, edited by H. Sawaya-Lacoste, vol. 517 of ESA Special Publication.

Rast, M. P. (2003b) The Scales of Granulation, Mesogranulation, and Supergranulation. ApJ, 597, 1200-1210.

Rempel, M. \& Schlichenmaier, R. (2011) Sunspot Modeling: From Simplified Models to Radiative MHD Simulations. Living Reviews in Solar Physics, 8, 3.

Rieutord, M. \& Rincon, F. (2010) The Sun's Supergranulation. Living Reviews in Solar Physics, 7, 2.

Rieutord, M., Roudier, T., Ludwig, H.-G., Nordlund, Å. \& Stein, R. (2001) Are granules good tracers of solar surface velocity fields? AEA, 377, L14-L17.

Rieutord, M., Roudier, T., Malherbe, J. M. \& Rincon, F. (2000) On mesogranulation, network formation and supergranulation. AEFA, 357, 1063-1072.

Rieutord, M., Roudier, T., Rincon, F., Malherbe, J.-M., Meunier, N., Berger, T. \& Frank, Z. (2010) On the power spectrum of solar surface flows. AEAA, 512, A4. 
RodDier, F. (1975) Principle of production of an acoustic hologram of the solar surface. Academie des Sciences Paris Comptes Rendus Serie B Sciences Physiques, 281, 93-95.

Rosenthal, C. S. \& Christensen-Dalsgaard, J. (1995) The interfacial F mode in a spherical solar model. MNRAS, 276, 1003-1008.

Rosenthal, C. S. \& Gough, D. O. (1994) The solar f-mode as an interfacial mode at the chromosphere-corona transition. ApJ, 423, 488-495.

Roudier, T., Švanda, M., Rieutord, M., Malherbe, J. M., Burston, R. \& Gizon, L. (2014) Structure and evolution of solar supergranulation using SDO/HMI data. AEFA, 567, A138.

Rüdiger, G., Brandenburg, A. \& PipIn, V. V. (1999) A helicity proxy from horizontal solar flow patterns. Astronomische Nachrichten, 320, 135.

Rüdiger, G., KÜKer, M. \& Tereshin, I. (2014) The existence of the $\Lambda$ effect in the solar convection zone as indicated by SDO/HMI data. $A \mathcal{E} A, \mathbf{5 7 2}, \mathrm{L} 7$.

Rybák, J., Wöhl, H., Kučera, A., Hanslmeier, A. \& Steiner, O. (2004) Indications of shock waves in the solar photosphere. AEFA, 420, 1141-1152.

Savitzky, A. \& Golay, M. J. E. (1964) Smoothing and Differentiation of Data by Simplified Least Squares Procedures. Analytical Chemistry, 36, 1627-39.

Scherrer, P. H., Bogart, R. S., Bush, R. I., Hoeksema, J. T., Kosovichev, A. G., Schou, J., Rosenberg, W., Springer, L. ET AL. (1995) The Solar Oscillations Investigation Michelson Doppler Imager. Sol. Phys., 162, 129-188.

Scherrer, P. H., Schou, J., Bush, R. I., Kosovichev, A. G., Bogart, R. S., Hoeksema, J. T., Liu, Y., Duvall, T. L. ET Al. (2012) The Helioseismic and Magnetic Imager (HMI) Investigation for the Solar Dynamics Observatory (SDO). Sol. Phys., 275, 207-227.

Schou, J. (2003) Wavelike Properties of Solar Supergranulation Detected in Doppler Shift Data. ApJ, 596, L259-L262.

Schou, J., Antia, H. M., Basu, S., Bogart, R. S., Bush, R. I., Chitre, S. M., ChristensenDalsgaArd, J., Di Mauro, M. P. et Al. (1998) Helioseismic Studies of Differential Rotation in the Solar Envelope by the Solar Oscillations Investigation Using the Michelson Doppler Imager. ApJ, 505, 390-417.

Schou, J., Scherrer, P. H., Bush, R. I., Wachter, R., Couvidat, S., Rabello-Soares, M. C., Bogart, R. S., Hoeksema, J. T. et AL. (2012) Design and Ground Calibration of the Helioseismic and Magnetic Imager (HMI) Instrument on the Solar Dynamics Observatory (SDO). Sol. Phys., 275, 229-259.

SchwarzschiLd, M. (1975) On the scale of photospheric convection in red giants and supergiants. ApJ, 195, 137-144. 
Simon, G. W. \& Leighton, R. B. (1964) Velocity Fields in the Solar Atmosphere. III. Large-Scale Motions, the Chromospheric Network, and Magnetic Fields. ApJ, 140, 1120 .

Simon, G. W., November, L. J., Ferguson, S. H., Shine, R. A., Tarbell, T. D., Title, A. M., Topka, K. P. \& Zirin, H. (1989) Details of Large Scale Solar Motions Revealed by Granulation Test Particles. In NATO Advanced Science Institutes (ASI) Series C, edited by R. J. Rutten \& G. Severino, vol. 263 of NATO Advanced Science Institutes (ASI) Series $C$.

Simon, G. W. \& Weiss, N. O. (1997) Kinematic Modeling of Vortices in the Solar Photosphere. ApJ, 489, 960-967.

Snodgrass, H. B. (1984) Separation of large-scale photospheric Doppler patterns. Sol. Phys., 94, 13-31.

Snodgrass, H. B. \& Ulrich, R. K. (1990) Rotation of Doppler features in the solar photosphere. ApJ, 351, 309-316.

Solanki, S. K., del Toro Iniesta, J. C., Woch, J., Gandorfer, A., Hirzberger, J., Schmidt, W., Appourchaux, T., Alvarez-Herrero, A. et al. (2015) The Polarimetric and Helioseismic Imager for Solar Orbiter: SO/PHI. In IAU Symposium, vol. 305 of IAU Symposium.

SPIEgEl, E. A. \& ZAHN, J.-P. (1992) The solar tachocline. AEFA, 265, 106-114.

Spruit, H. (1997) Convection in stellar envelopes: a changing paradigm. Mem. Soc. Astron. Italiana, 68, 397.

Stein, R. F. (2012) Solar Surface Magneto-Convection. Living Reviews in Solar Physics, 9, 4 .

Stein, R. F., Benson, D., Georgobiani, D. \& Nordlund, A. (2006) Supergranule scale convection simulations. In Proceedings of SOHO 18/GONG 2006/HELAS I, Beyond the spherical Sun, vol. 624 of ESA Special Publication.

Stein, R. F., Lagerfjård, A., Nordlund, Å., Georgobiani, D., Benson, D. \& SchaffenBERGER, W. (2009) Supergranulation Scale Convection Simulations. In The Second Hinode Science Meeting: Beyond Discovery-Toward Understanding, edited by B. Lites, M. Cheung, T. Magara, J. Mariska \& K. Reeves, vol. 415 of Astronomical Society of the Pacific Conference Series.

Stein, R. F. \& Nordlund, A. (1989) Topology of convection beneath the solar surface. ApJ, 342, L95-L98.

Stein, R. F. \& NoRdlund, Å. (1998) Simulations of Solar Granulation. I. General Properties. ApJ, 499, 914-933.

Stein, R. F. \& Nordlund, ^. (2001) Solar Oscillations and Convection. II. Excitation of Radial Oscillations. ApJ, 546, 585-603. 
Stix, M. (2002) The Sun. 2nd edn., Springer, corrected second printing 2004.

TAYLoR, G. I. (1918) On the dissipation of eddies. Reports and Memoranda of the Advisory Committee for Aeronautics, 598, 73-78.

Thompson, M. J., Toomre, J., Anderson, E. R., Antia, H. M., Berthomieu, G., Burtonclay, D., Chitre, S. M., Christensen-Dalsgaard, J. et al. (1996) Differential Rotation and Dynamics of the Solar Interior. Science, 272, 1300-1305.

Toomre, J. (2002) Order Amidst Turbulence. Science, 296, 64-65.

Tsuneta, S., Ichimoto, K., Katsukawa, Y., Nagata, S., Otsubo, M., Shimizu, T., Suematsu, Y., NAKagiRI, M. ET al. (2008) The Solar Optical Telescope for the Hinode Mission: An Overview. Sol. Phys., 249, 167-196.

Turck-ChiEzze, S. \& Couvidat, S. (2011) Solar neutrinos, helioseismology and the solar internal dynamics. Reports on Progress in Physics, 74(8), 086901.

Verma, M., Steffen, M. \& Denker, C. (2013) Evaluating local correlation tracking using CO5BOLD simulations of solar granulation. AEFA, 555, A136.

Veronis, G. (1959) Cellular convection with finite amplitude in a rotating fluid. Journal of Fluid Mechanics, 5, 401-435.

Wachter, R., Schou, J., Rabello-Soares, M. C., Miles, J. W., Duvall, T. L. \& Bush, R. I. (2012) Image Quality of the Helioseismic and Magnetic Imager (HMI) Onboard the Solar Dynamics Observatory (SDO). Sol. Phys., 275, 261-284.

Wang, Y., Noyes, R. W., Tarbell, T. D. \& Title, A. M. (1995) Vorticity and Divergence in the Solar Photosphere. ApJ, 447, 419.

Welsch, B. T., Abbett, W. P., De Rosa, M. L., Fisher, G. H., Georgoulis, M. K., Kusano, K., Longcope, D. W., Ravindra, B. et aL. (2007) Tests and Comparisons of VelocityInversion Techniques. ApJ, 670, 1434-1452.

Woodard, M. F. (2002) Solar Subsurface Flow Inferred Directly from FrequencyWavenumber Correlations in the Seismic Velocity Field. ApJ, 565, 634-639.

Yeo, K. L., Feller, A., Solanki, S. K., Couvidat, S., Danilovic, S. \& Krivova, N. A. (2014) Point spread function of SDO/HMI and the effects of stray light correction on the apparent properties of solar surface phenomena. $A \mathcal{E} A, \mathbf{5 6 1}, \mathrm{A} 22$.

Zeldovich, Y. B., Ruzmaikin, A. A. \& Sokoloff, D. D. (1990) Magnetic Fields in Astrophysics. Gordon and Breach.

Zhao, J., Bogart, R. S., Kosovichev, A. G., Duvall, Jr., T. L. \& Hartlep, T. (2013) Detection of Equatorward Meridional Flow and Evidence of Double-cell Meridional Circulation inside the Sun. ApJ, 774, L29.

Zhao, J. \& Kosovichev, A. G. (2003) On the inference of supergranular flows by timedistance helioseismology. In GONG+2002. Local and Global Helioseismology: the Present and Future, edited by H. Sawaya-Lacoste, vol. 517 of ESA Special Publication. 
Appendices 



\section{A Time-distance measurements}

\section{A.1 Ridge filter construction}

As a supplement to Sect. 3.8, some further details on the construction of the ridge filters are given in the following.

The filters for HMI are based on ridge maxima that were identified from an azimuthally averaged, 60-day average of $8 \mathrm{~h}$ Dopplergram datasets that cross the central

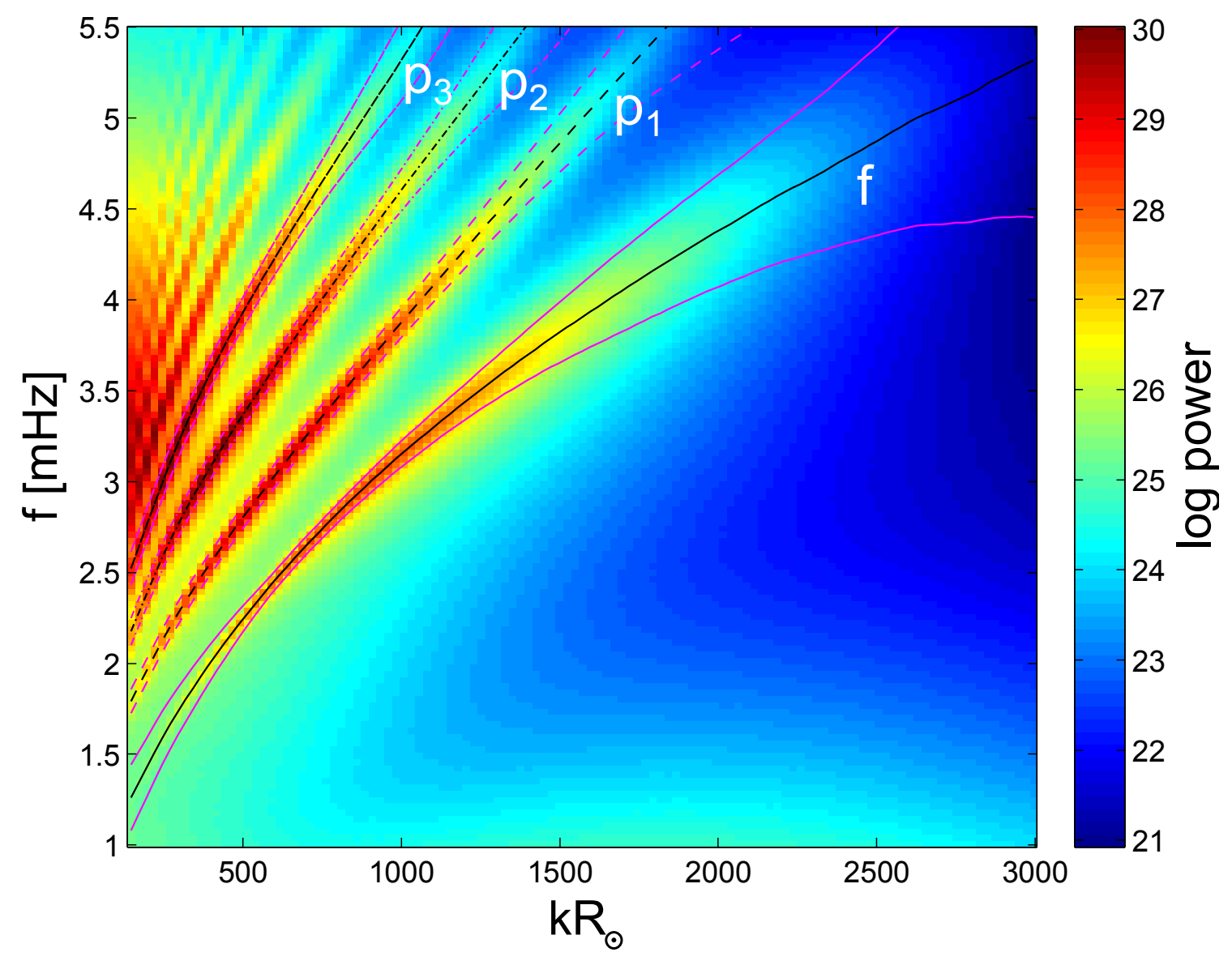

Figure A.1: Identified ridge maxima in power spectrum of HMI Dopplergrams. The power spectrum was computed for individual $8 \mathrm{~h}$ datasets observed at the solar equator in 2012 and averaged over azimuth and 60 days. The black lines denote the identified frequencies of the ridge maxima after smoothing for the $\mathrm{f}, \mathrm{p}_{1}, \mathrm{p}_{2}$, and $\mathrm{p}_{3}$ modes as a function of $k R_{\odot}$. The magenta lines show the symmetrized FWHM of the ridge peaks. 
meridian at the solar equator in 2012. The tracking parameters are analogous to the ones for the 2010 data described elsewhere in this thesis. To determine the location of the ridge maxima, for each $k$ the peaks in power that are higher than any of the $n_{n}$ neighboring frequency pixels on each side are identified. The value of $n_{n}$ is chosen to be larger than one to prevent the selection of maxima that arise due to noise. On the other hand, a too high value of $n_{n}$ would thwart the detection of closely spaced ridge maxima, especially at low wavenumbers. Typical values for HMI and an observation time $T=8 \mathrm{~h}$ are $n_{n}=4$ if $k R_{\odot}<2000$ and $n_{n}=7$ elsewhere. For MDI, the parameter $n_{n}$ is set to the same value (except $n_{n}=3$ for $k R_{\odot}<200$ ). The search for ridge maxima is confined to a frequency interval that excludes the convective noise at low frequencies. In addition, the FWHM $\omega_{\Gamma}$ of the power is measured around the identified ridge maxima. In a next step, the precision of the peak locations is improved by calculating the vertex of the parabola that includes the maximum and the adjacent pixels. To further reduce the scatter of the maxima positions and the FWHM, the identified ridge curves $\omega_{\text {mode }}(k)$ and $\omega_{\Gamma}(k)$ are smoothed by a moving-average filter in $k$. The power spectrum and the identified ridge curves for HMI are shown in Fig. A.1.

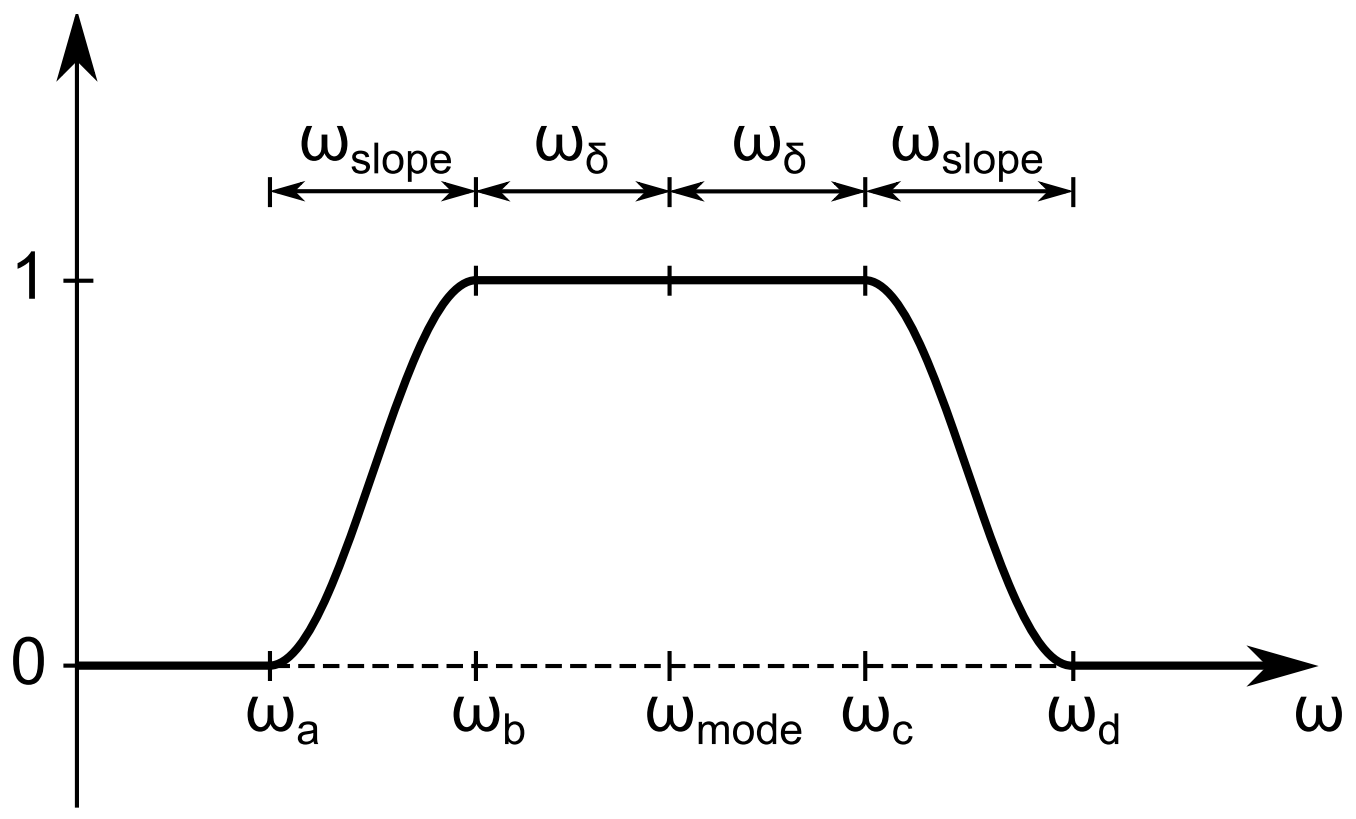

Figure A.2: Sketch of ridge filter structure. The filter acts in Fourier space for a particular wavenumber $k$ as a function of angular frequency $\omega$. The filter consists of a plateau region of width $2 \omega_{\delta}$, centered around the ridge maximum $\omega_{\text {mode }}(k)$. On both sides, a raised cosine of width $\omega_{\text {slope }}$ guides the filter down to the zero level. The limits of the plateau and transition regions are labeled (in order of increasing frequency) $\omega_{a}, \omega_{b}, \omega_{c}$, and $\omega_{d}$.

The filters are then constructed symmetrically around $\omega_{\text {mode }}$ for each $k$, as described in Sect. 3.8. A sketch of the filter structure for a given wavenumber is shown in Fig. A.2. 
The filter limits are given by

$$
\begin{aligned}
& \omega_{a}=\omega_{\text {mode }}-\omega_{\delta}-\omega_{\text {slope }} \\
& \omega_{b}=\omega_{\text {mode }}-\omega_{\delta} \\
& \omega_{c}=\omega_{\text {mode }}+\omega_{\delta} \\
& \omega_{d}=\omega_{\text {mode }}+\omega_{\delta}+\omega_{\text {slope }}
\end{aligned}
$$

$$
\begin{array}{r}
\text { (lower filter limit) } \\
\text { (lower plateau limit) } \\
\text { (upper plateau limit) } \\
\text { (upper filter limit) }
\end{array}
$$

Figure A.3 shows the filter limits for HMI superposed on the average power spectrum. The filters are cut to zero left of the left vertical line and right of the right vertical line. Beyond these limits, the excess power of the ridge peak is less than the background power. The effect of the filters is demonstrated in Fig. A.4. The power is shown at the f- and $p_{1^{-}}$ mode ridges before and after filtering for cuts at different wavenumbers that span the whole filter range. The filters select the desired mode ridge while bringing the power down to zero smoothly without capturing much power of the neighboring ridges.

To evaluate if the filters are still able to capture the right ridges in the presence of a uniform flow $\mathbf{u}$ in the $x$ direction, we applied Doppler shifts $\delta \omega=\mathbf{k} \cdot \mathbf{u}$ to an average power spectrum, as described in Sect. 3.9. The power for $u_{x}=-500,0$, and $500 \mathrm{~m} \mathrm{~s}^{-1}$ at selected $k_{x} R_{\odot}$ values is shown for both $\mathrm{f}$ and $\mathrm{p}_{1}$ modes in Fig. A.5. Most of the ridge power is still captured for these Doppler shifts at the given wavenumbers.

\section{A.2 Correlation of rotated $\tau^{\mathrm{ac}}$ measurements}

As discussed in Sect. 2.3.3, the vorticity-sensitive $\tau^{\text {ac }}$ measurements are highly correlated for small rotation angles $\beta$. As a consequence, the $\mathrm{S} / \mathrm{N}$ cannot be substantially improved for increasing the number of angles $n_{\beta}$ beyond some point. This was illustrated by the variance of $\tau^{\mathrm{ac}}$ reaching a plateau in Fig. $2.4 \mathrm{~b}$ at about $n_{\beta}=8$. The corresponding successive rotation angle is $\Delta \beta=60^{\circ} / 8=7.5^{\circ}$.

To underline this statement, we calculated the correlation coefficient for $\tau^{\text {ac }}$ measurements that are separated by an angle $\Delta \beta$, $\operatorname{Corr}\left[\tau^{\mathrm{ac}}\left(\beta_{0}\right), \tau^{\mathrm{ac}}\left(\beta_{0}+\Delta \beta\right)\right]$, for $\beta_{0}=0$ (Fig. A.6). Indeed the correlation coefficient decreases below 0.1 for $\Delta \beta=7.5^{\circ}$. For $\Delta \beta=2.5^{\circ}$, the $\tau^{\text {ac }}$ measurements are highly correlated. Note that because of the hexagonal symmetry of the measurement, $\operatorname{Corr}\left[\tau^{\mathrm{ac}}\left(0^{\circ}\right), \tau^{\mathrm{ac}}(\Delta \beta)\right]=\operatorname{Corr}\left[\tau^{\mathrm{ac}}\left(0^{\circ}\right), \tau^{\mathrm{ac}}\left(\Delta \beta+60^{\circ}\right)\right]$, i.e., rotating the measurement structure by $60^{\circ}$ yields the same measurement. In addition, the correlation is symmetric with respect to $\Delta \beta=30^{\circ}$. This means that it does not matter for the correlation whether the $\tau^{\mathrm{ac}}$ measurement structure is rotated clockwise or anti-clockwise. 

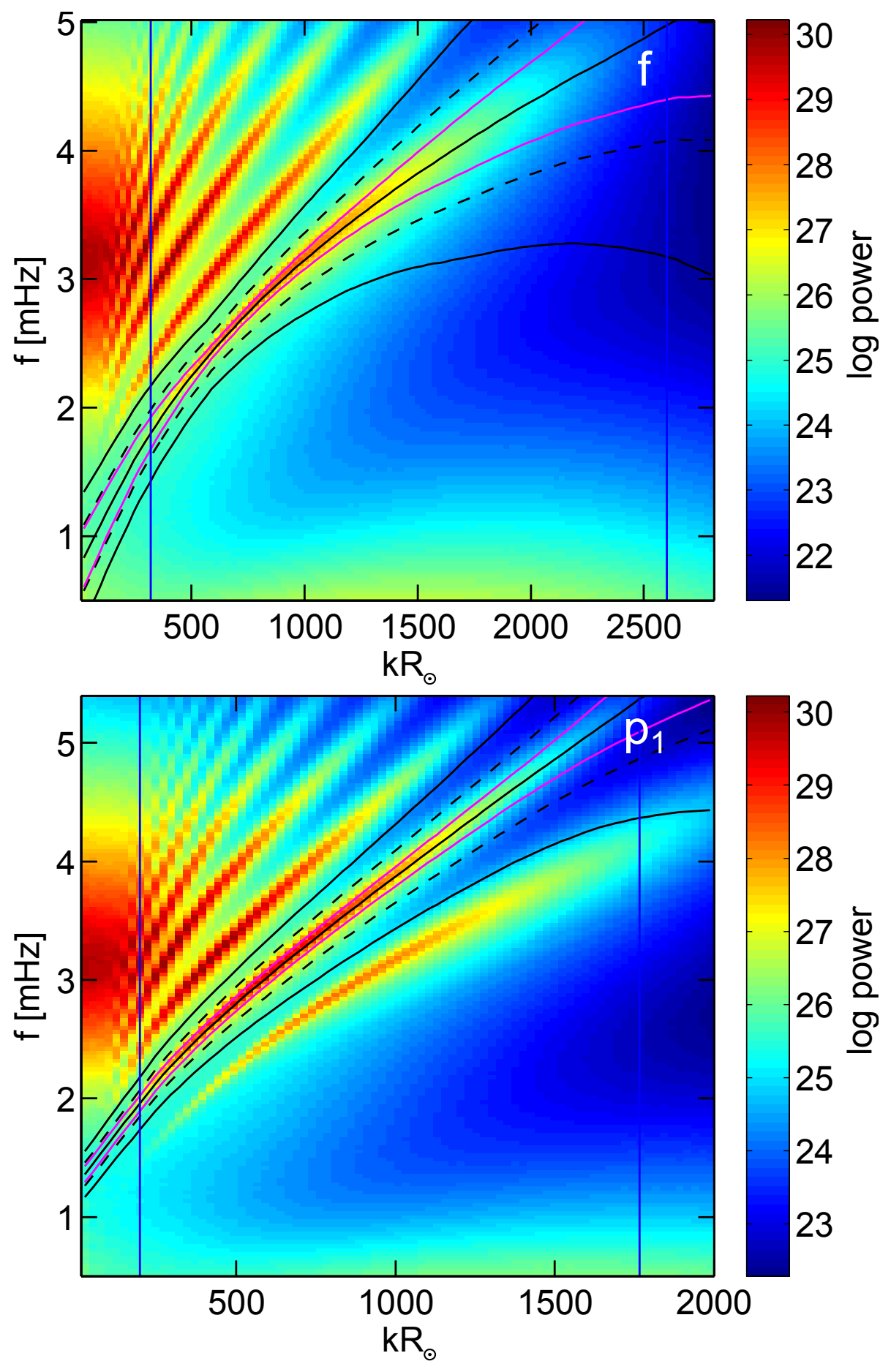

Figure A.3: Ridge filter contours in power spectrum of HMI Dopplergrams. Top: $\mathrm{f}$ modes. Bottom: $p_{1}$ modes. The power in both panels was averaged over azimuth and 60 days of HMI $8 \mathrm{~h}$ Dopplergrams observed at the solar equator in 2012. The ridge centers and filter limits are described by the solid black lines. The dashed lines give the edges of the filter plateau, and the magenta lines indicate the FWHM. The vertical lines show the filter limits in wavenumber (corresponding to $\omega_{\min }$ and $\omega_{\max }$ in Table 3.2). 

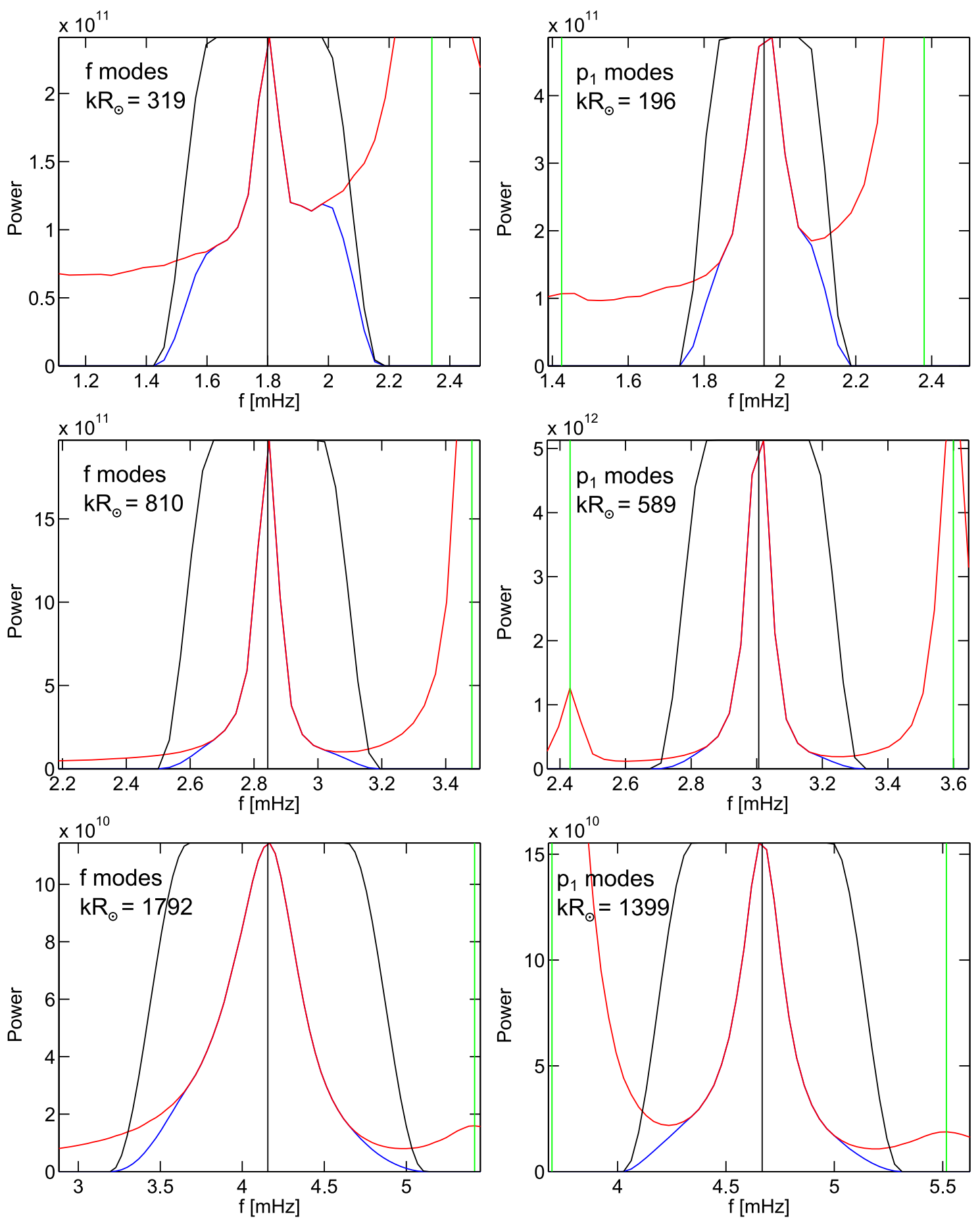

Figure A.4: Cuts of filters, original and filtered power spectrum for different $k R_{\odot}$ values. Left column: f modes. Right column: $p_{1}$ modes. The black curves show the filters (top axis corresponds to filter value 1, bottom axis to filter value 0 ), the red curves show the unfiltered power, and the blue curves show the filtered power. The vertical black and green lines denote the identified maxima of the respective mode ridge and the neighboring ridges. 

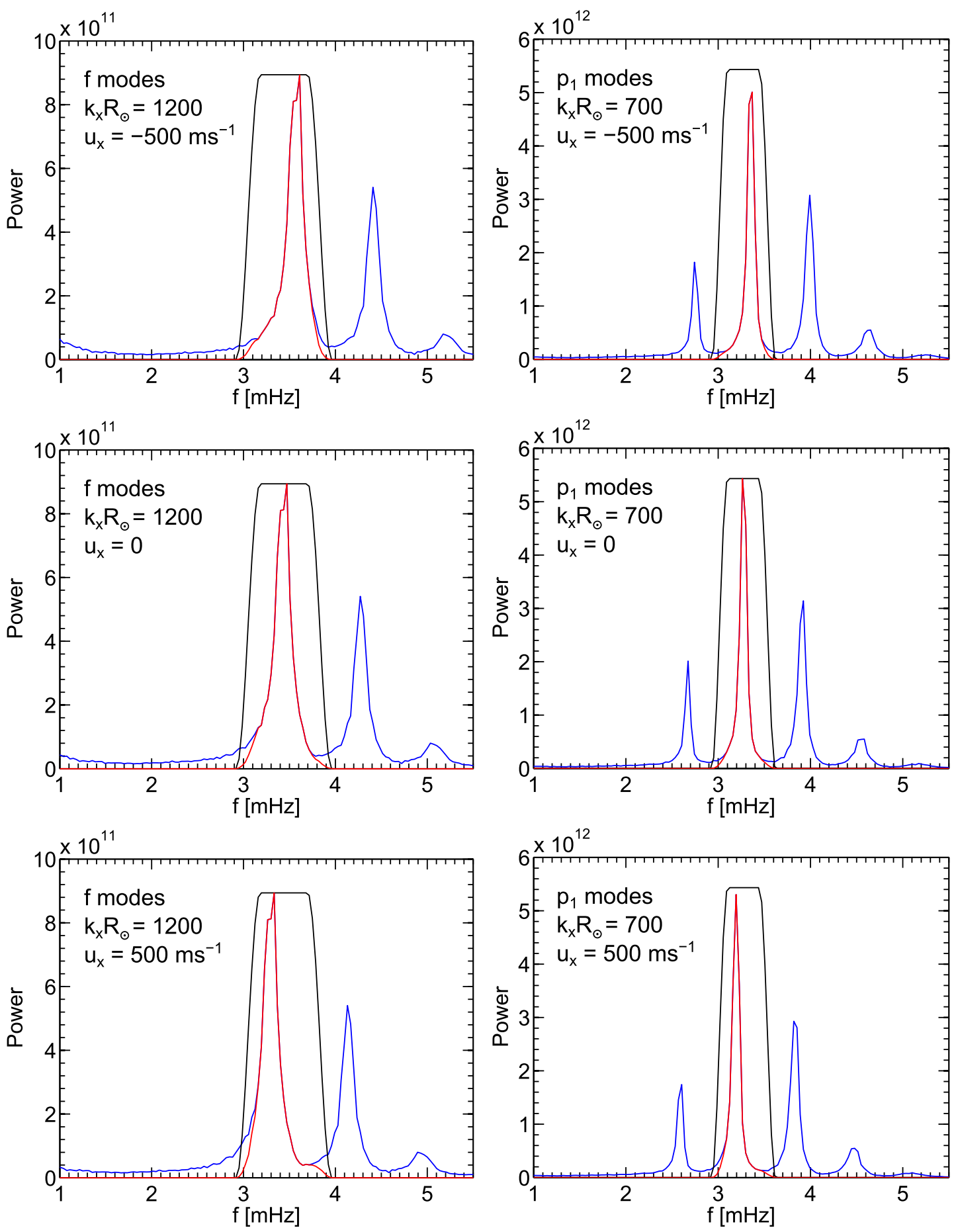

Figure A.5: Cuts of Doppler-shifted power spectrum of Dopplergrams at $k_{y}=0$ for different flow velocities in the $x$ direction. Left column: $\mathrm{f}$ modes, cut at $k_{x} R_{\odot}=1200$. Right column: $\mathrm{p}_{1}$ modes, cut at $k_{x} R_{\odot}=700$. The power before and after filtering is shown by the blue and red curves. The black curves show the filter (rescaled to the ridge maximum). The original power spectrum $\left(u_{x}=0\right)$ is an average over 60 power spectra of $8 \mathrm{~h}$ Doppler datasets at the solar equator in 2010 (not azimuthally averaged). 
(a)
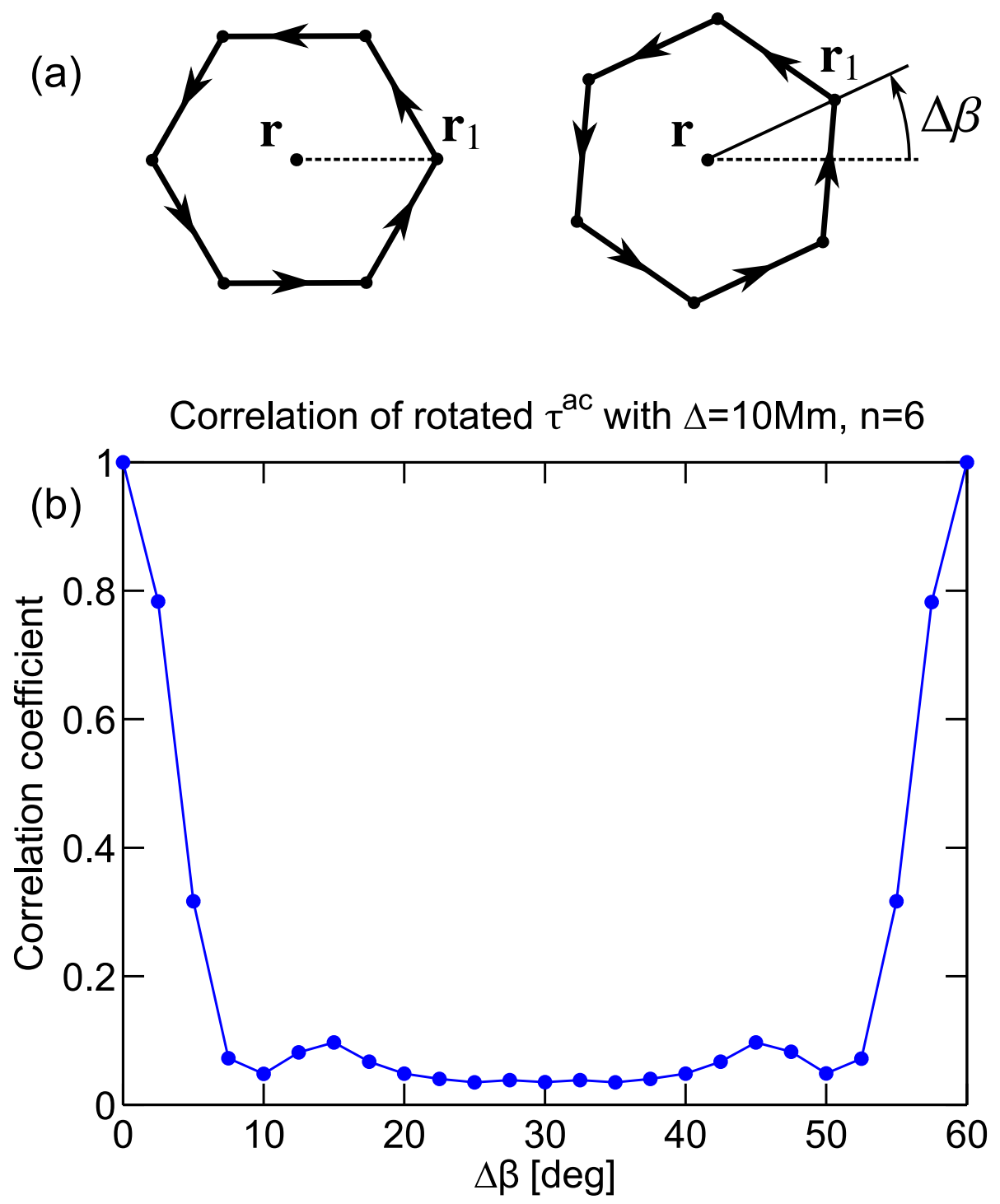

Figure A.6: a) Pair of $\tau^{\text {ac }}$ measurements for $n=6$ and $\Delta=10 \mathrm{Mm}$, with fixed $\beta=0^{\circ}$ for the first measurement (left) and varying $\beta=\Delta \beta$ for the second measurement (right). b) Correlation of $\tau^{\mathrm{ac}}$ measurements shown in a) as a function of $\Delta \beta$. The spacing of $\Delta \beta$ in this plot is $2.5^{\circ}$. 



\section{B Velocity and vorticity measurements}

\section{B.1 Center-to-limb systematics}

As a supplement for Sect. 3.4 and Sect. 3.10.1, the analogues of Figs. 3.6 and Figs. 3.9 when not applying the empirical correction for center-to-limb systematics are provided.

In addition, the vorticity as depicted in Fig. 3.12 in Sect. 3.5 is shown for all analyzed latitudes, both after and before correction for center-to-limb systematics. 
B Velocity and vorticity measurements

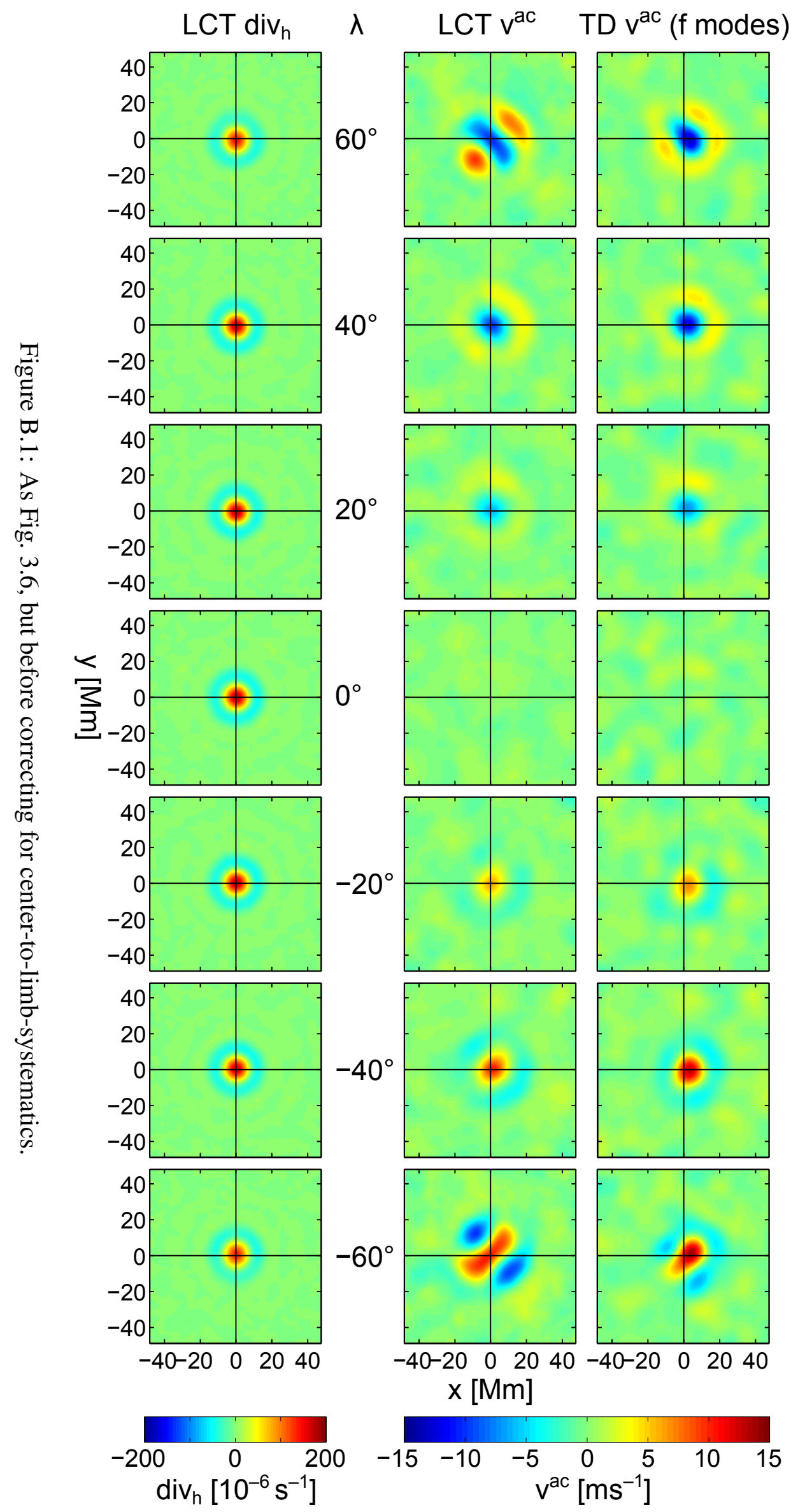


B.1 Center-to-limb systematics

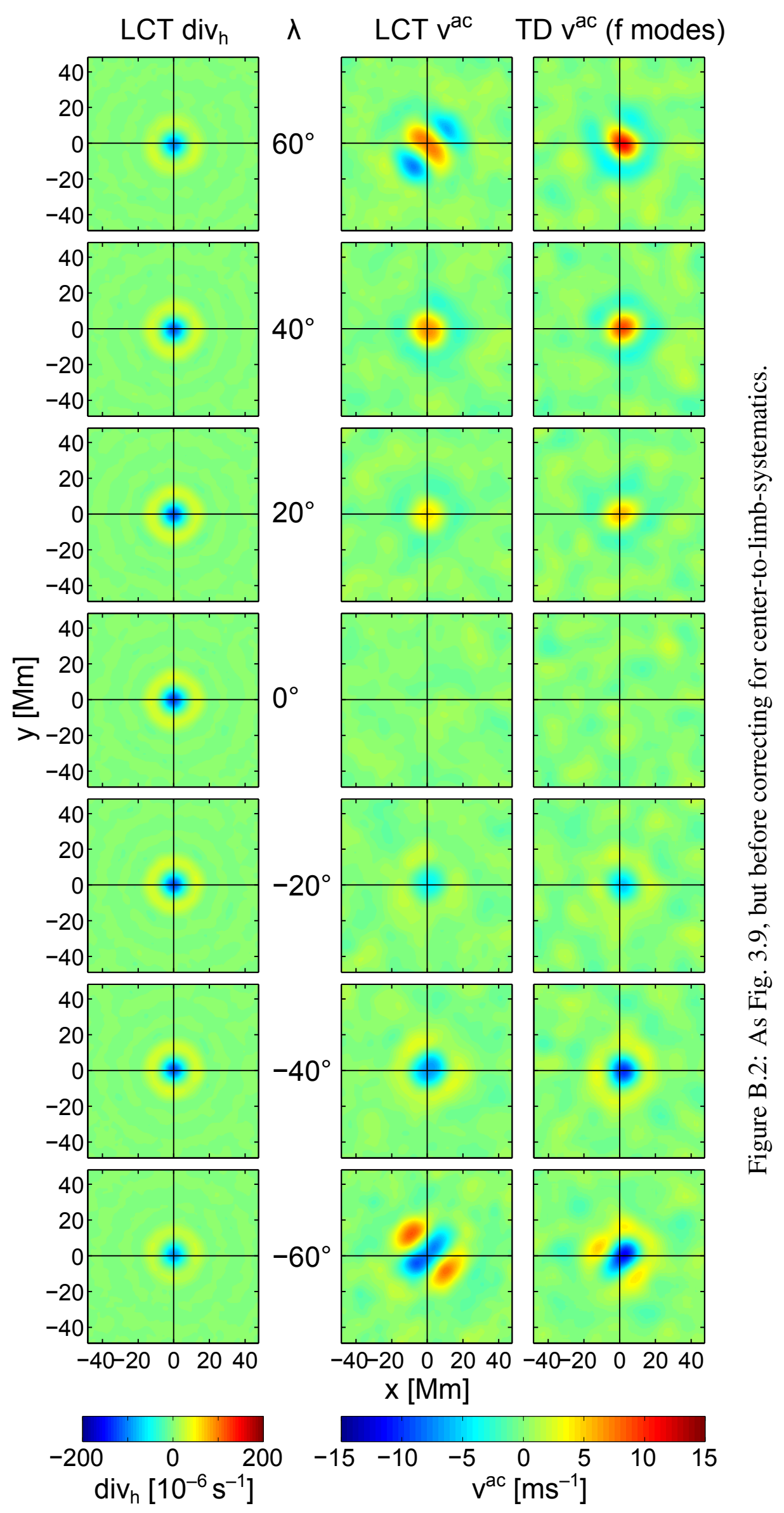




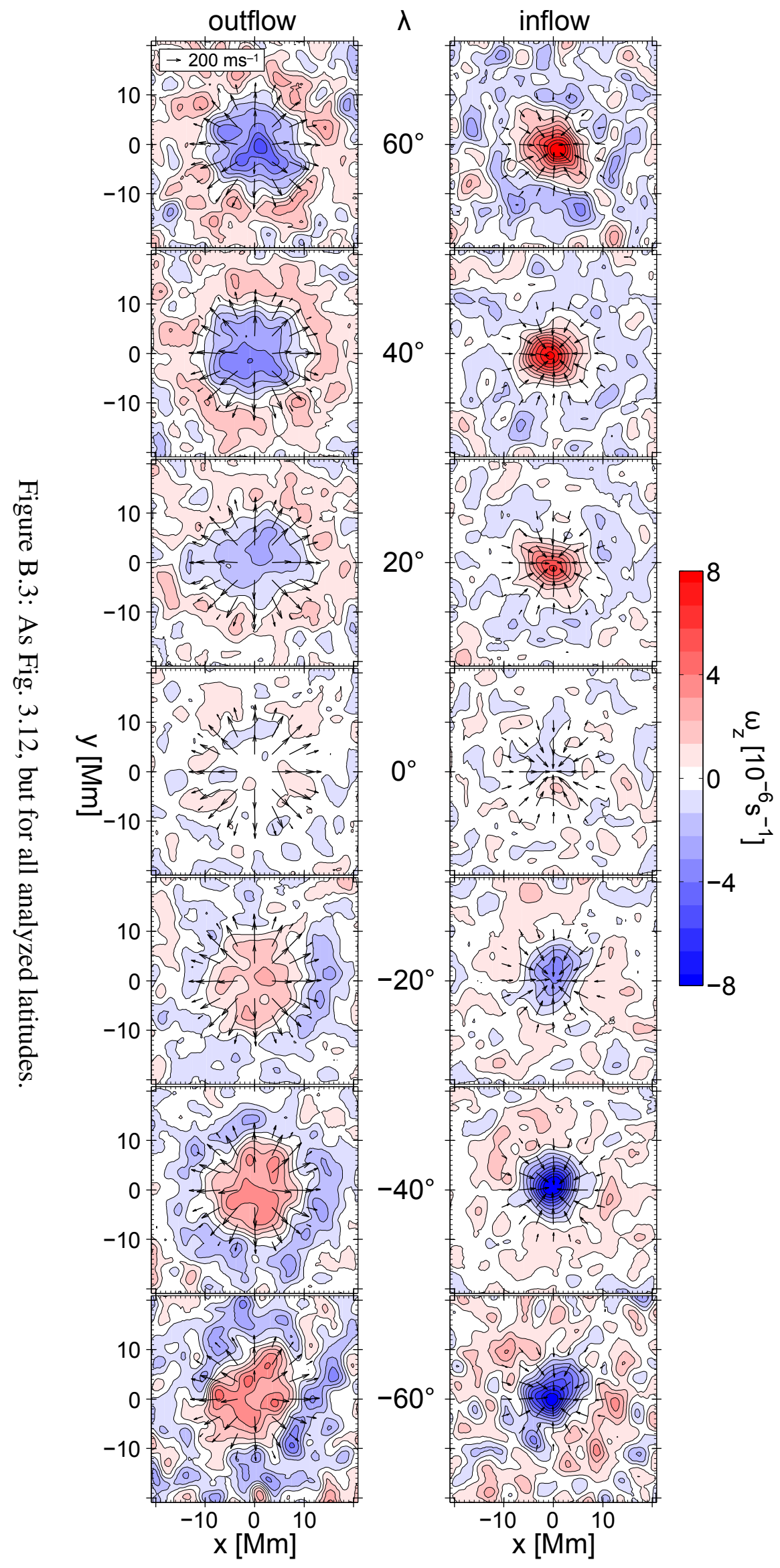



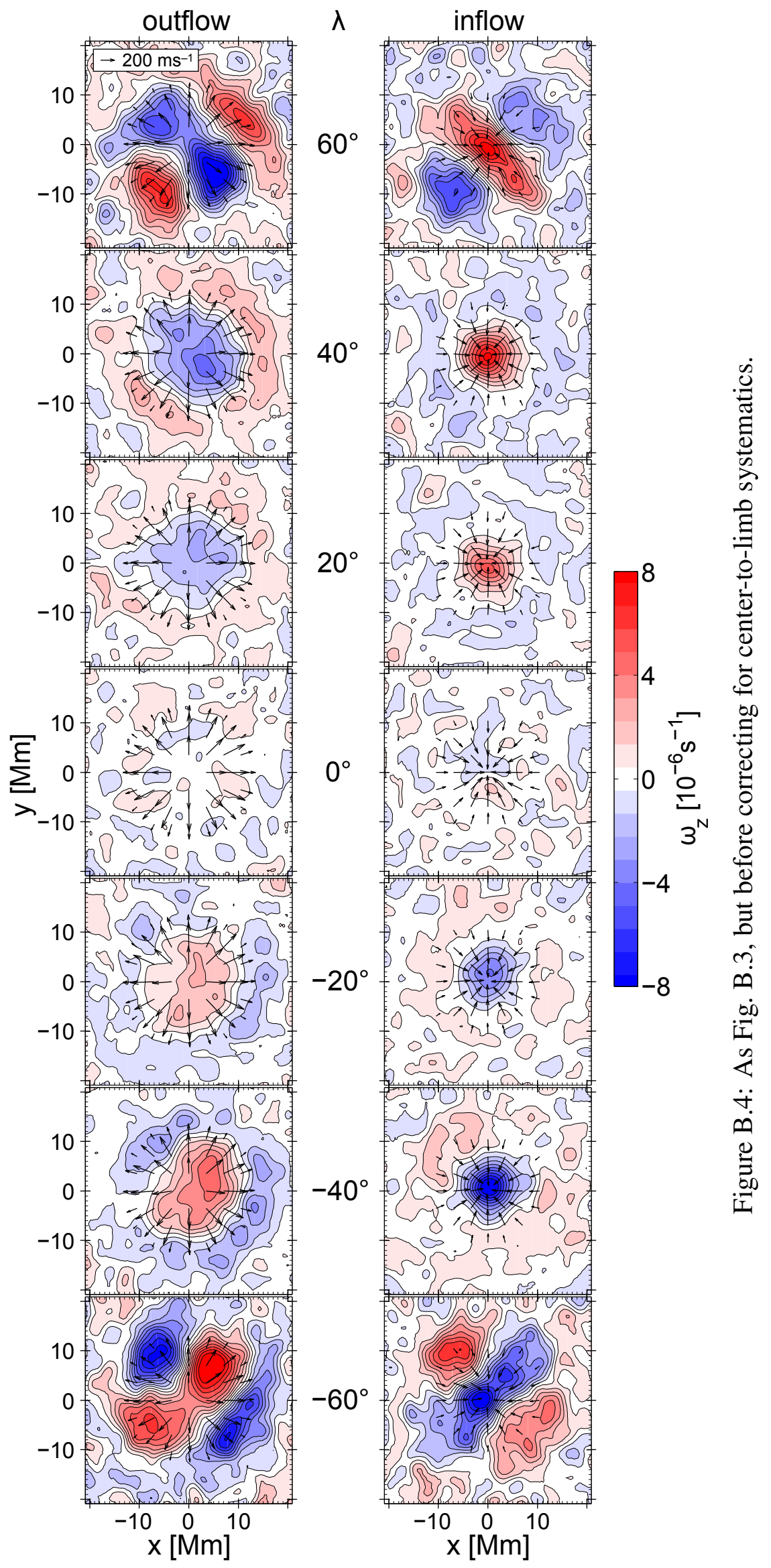



\section{B.2 Average supergranular outflow and inflow profiles}

Figure B.5 shows the azimuthally averaged horizontal velocity components (radial velocity $v_{r}$ and circular velocity $v_{t}$ ) of the average supergranular outflow and inflow (obtained from LCT) at all analyzed latitudes. In addition, the toy model relation

$$
v_{t}(r)=-r \Omega(\lambda) \sin \lambda
$$

that is discussed in Sect. 5.1 is overplotted on the measured $v_{t}$ curves for the average outflow. For all latitudes, the model is in agreement with the measurements for $r<5 \mathrm{Mm}$. 
B Velocity and vorticity measurements
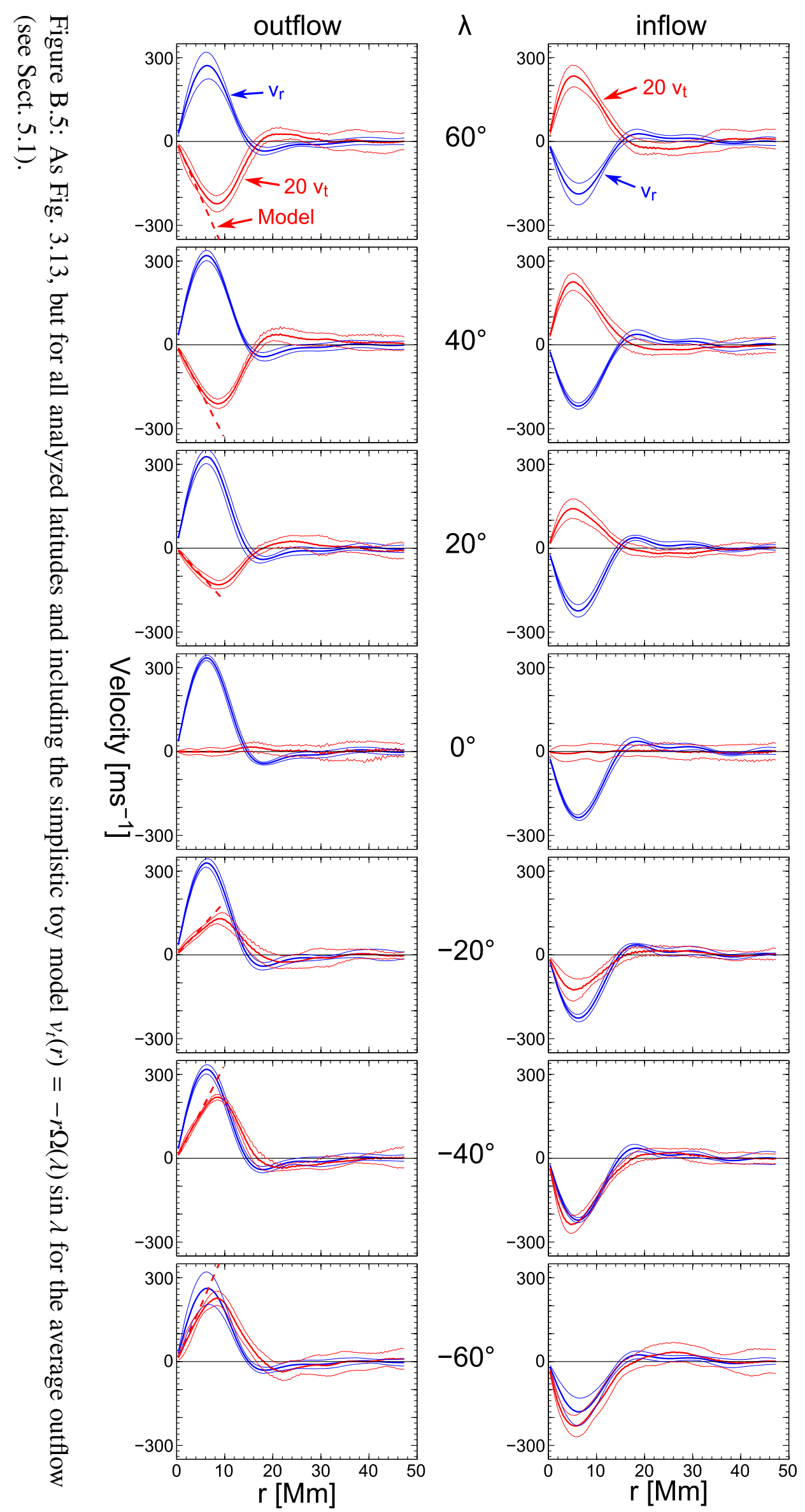


\section{Magnetic field measurements}

\section{C.1 Location of the network magnetic field}

Figure C.1 shows the line-of-sight magnetic field at the equator in relation to supergranules as they appear in TD and LCT measurements based on HMI observations. The strong magnetic features are clearly preferentially located in the inflows. The plot also visualizes the strong correlation between TD and LCT measurements that was discussed in Sect. 3.3.

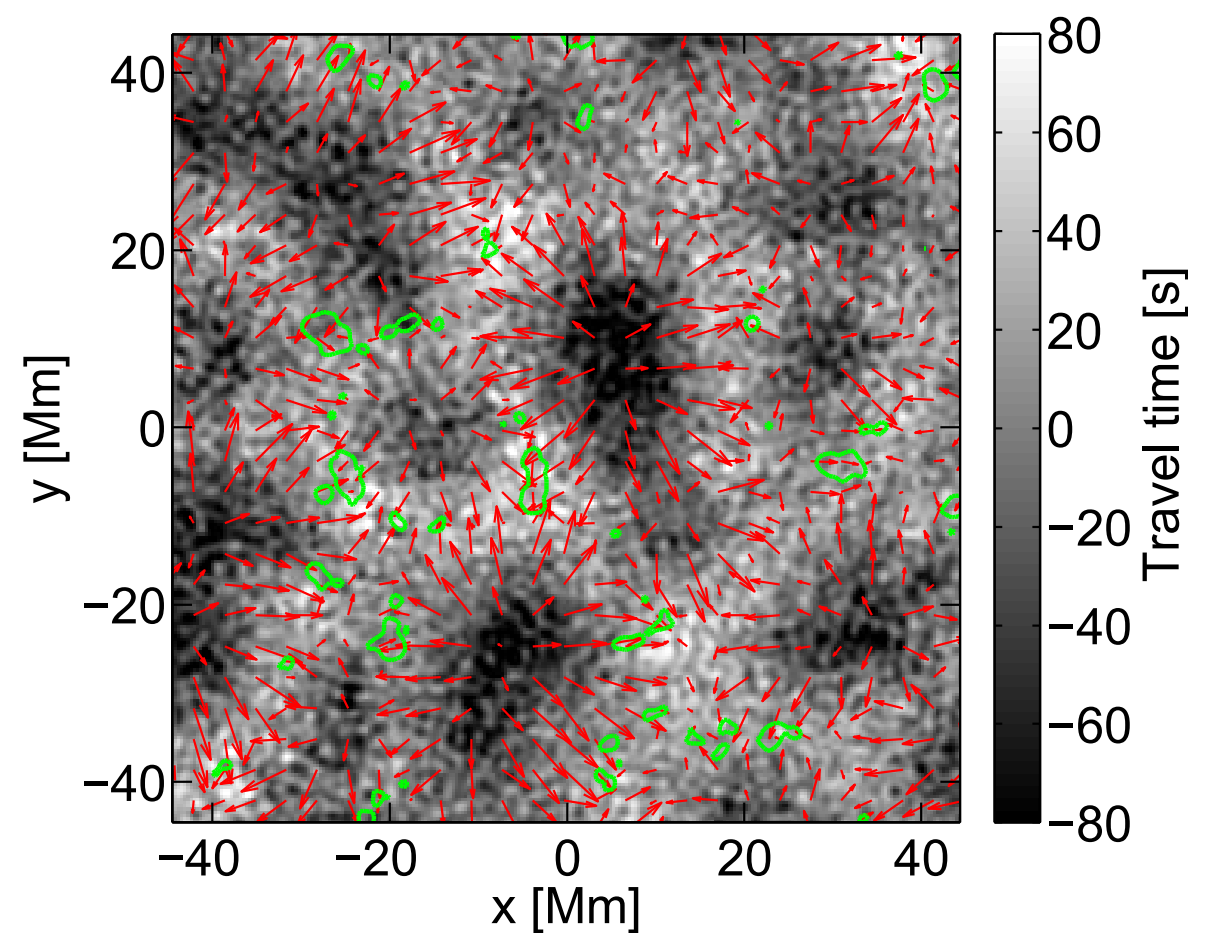

Figure C.1: Supergranular flows and network magnetic field as derived from TD, LCT, and line-of-sight magnetograms at the solar equator for HMI data. The grayscale colors show the f-mode-filtered $\tau^{\text {oi }}$ travel times. The shown region is one quarter of the full travel-time map area. The color bar was truncated to increase the contrast (the minimum and maximum travel times are $-149.3 \mathrm{~s}$ and $125.1 \mathrm{~s}$ ). The red arrows represent the horizontal flow velocity measured from local correlation tracking of granules. Additionally, the 20 Gauss level of the unsigned line-of-sight component of the magnetic field is shown as the green contours. All data shown have been averaged over 8 hours on 2 May 2010. 


\section{C.2 Relation between magnetic field and mean travel times}

As discussed in Sect.4.3.2, the relationship between the magnetic field $B$ and mean travel times $\delta \tau$ in the average supergranular outflow near disk center can roughly be described by the following relation:

$$
\delta \tau \sim \delta \tau_{\max }-\left(1.2 \mathrm{~s} \mathrm{Gauss}^{-1 / 2}\right)\left(B-B_{\min }\right)^{1 / 2},
$$

with $B_{\min }=1.3$ Gauss and $\delta \tau_{\max }=1.8 \mathrm{~s}$. In Fig. C.2, this relation is shown in a scatter plot for $B$ and $\delta \tau$. The left-hand part of the plot (weak $B$ ) contains points near the center of the supergranular outflow, whereas the right-hand part (strong $B$ ) comprises points in the surrounding ring of inflows.

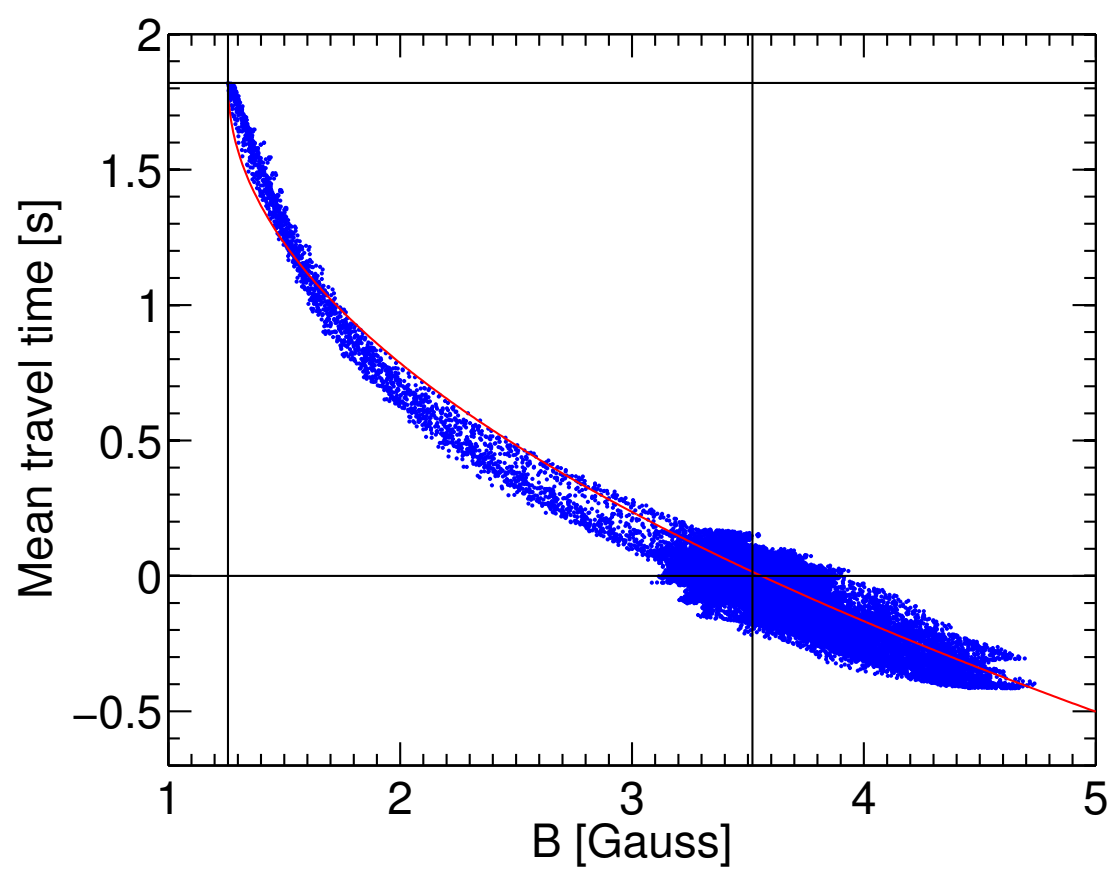

Figure C.2: Scatter plot showing mean travel times versus magnetic field $B$ in the average supergranule near disk center (see Fig. 4.2). The red line is the square-root relation defined in Eq. (C.1). The black lines mark the magnetic field strength and the mean travel time in the outflow center (upper left) and the mean values (lower right).

\section{C.3 Center-to-limb systematics}

In the following, center-to-limb systematics in the magnetic field and mean travel times are shown for the average supergranule as observed with HMI. This is accomplished by moving away from disk center eastward and westward (Fig. C.3). A straight average of the map pairs at $20^{\circ}, 40^{\circ}$, and $60^{\circ}$ east and west of the central meridian (Figs. C.4 and C.5) removes the center-to-limb systematics, while preserving the anisotropy that was detected at disk center (Fig. C.6. Note that the magnetic field was divided by the cosine of the heliocentric angle to account for the different projection angles (assuming that the network magnetic field around the average supergranule is predominantly vertical). 


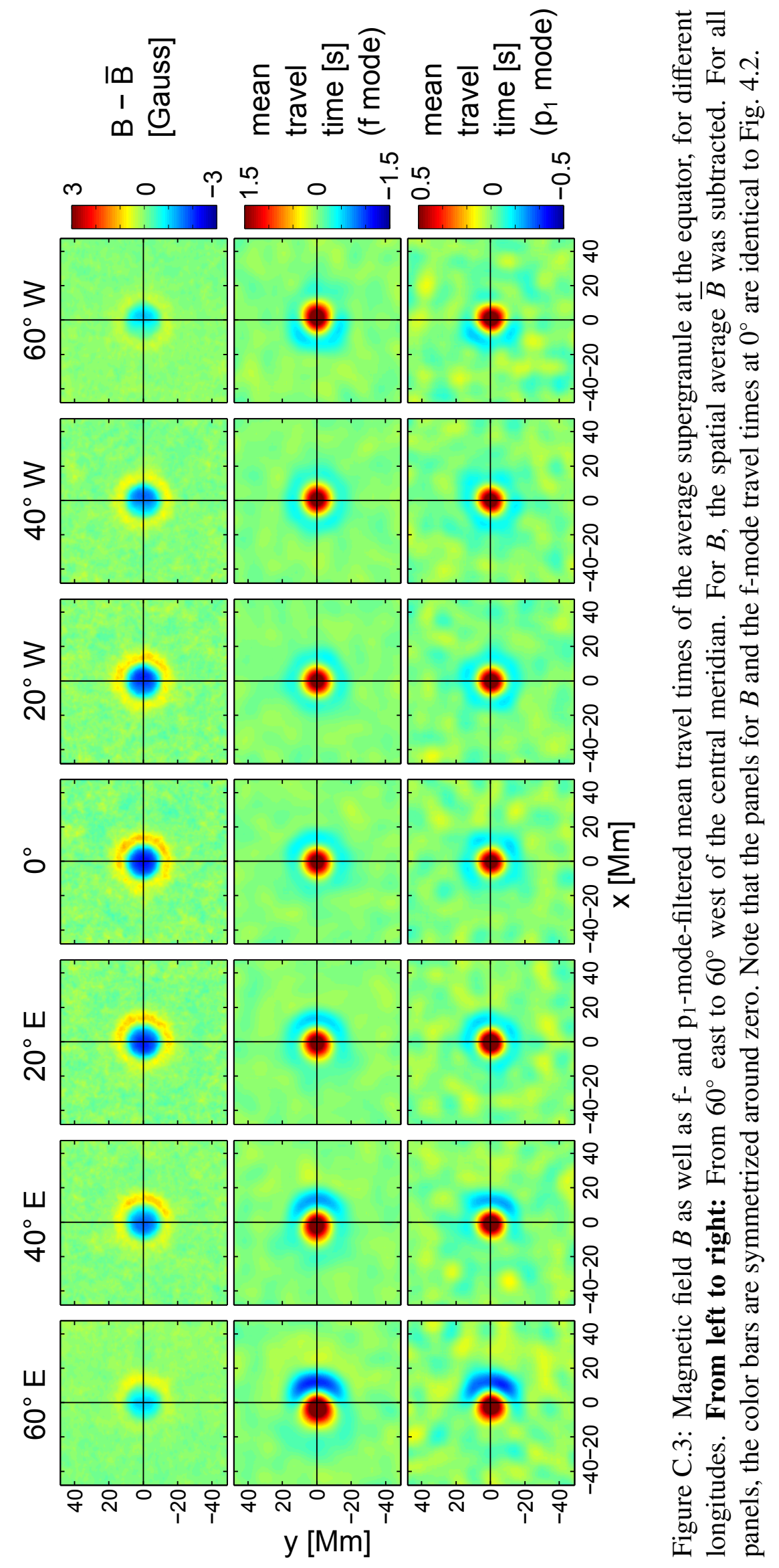



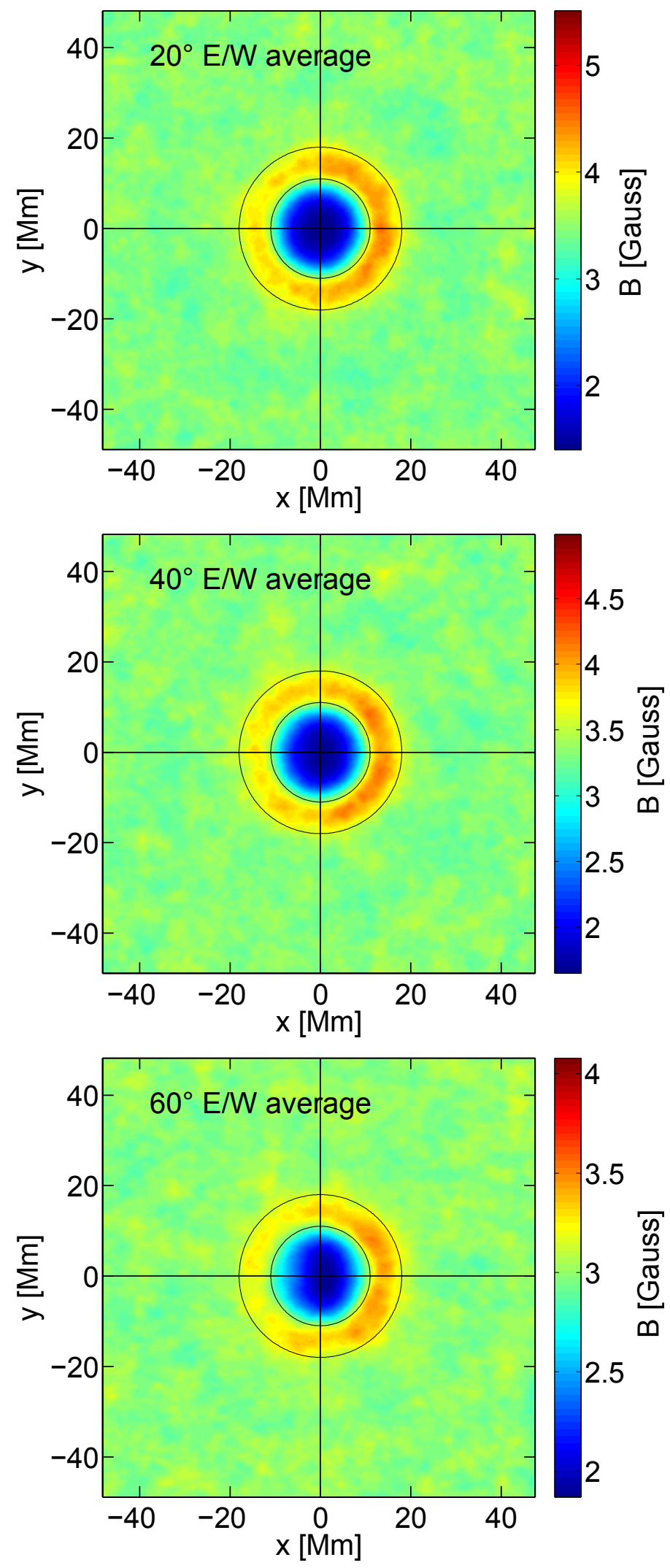

Figure C.4: Magnetic field $B$ like in the left panel of Fig. 4.2, but for averaging map pairs east and west of the central meridian. The color bars are symmetrized around the mean values (3.46, 3.32, and 2.97 Gauss). 

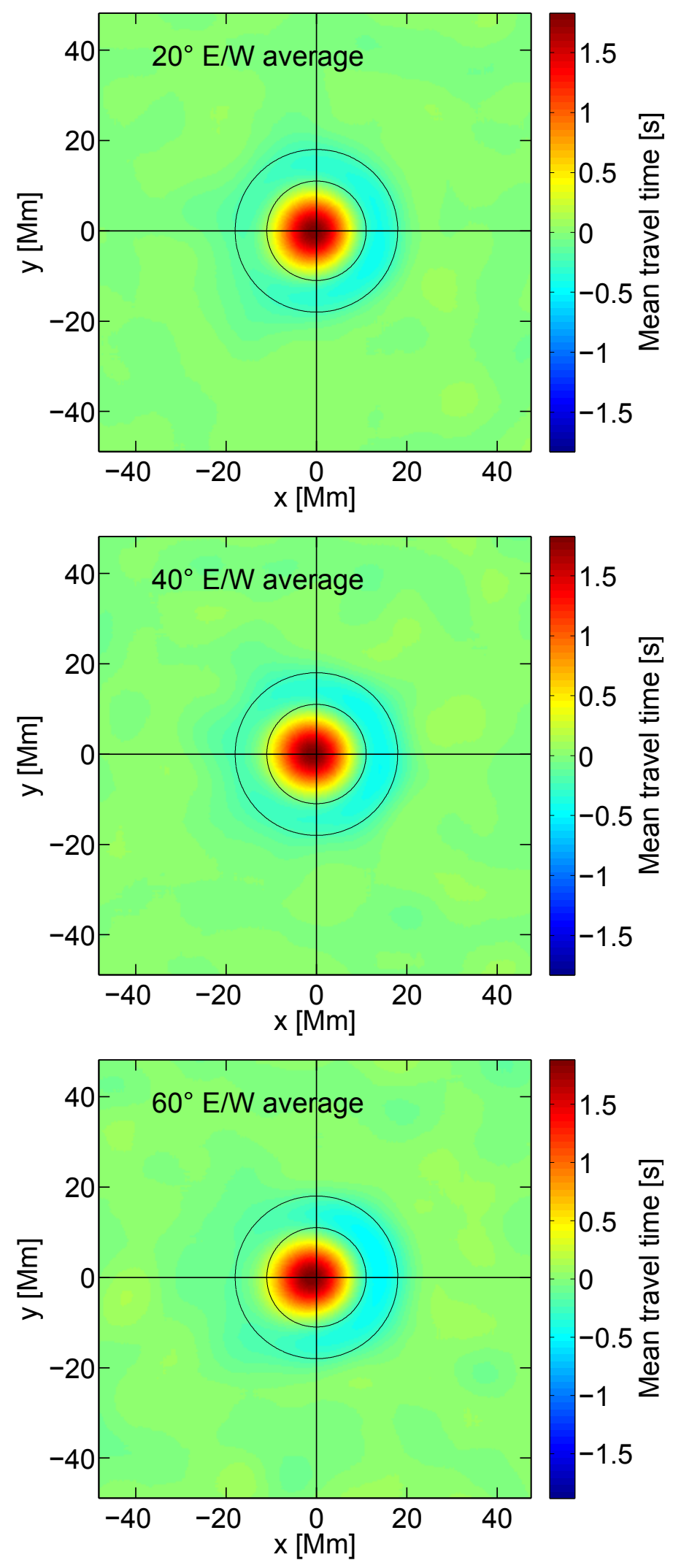

Figure C.5: Mean travel times like in the right panel of Fig. 4.2, but for averaging map pairs east and west of the central meridian. The color bars are symmetrized around zero. 

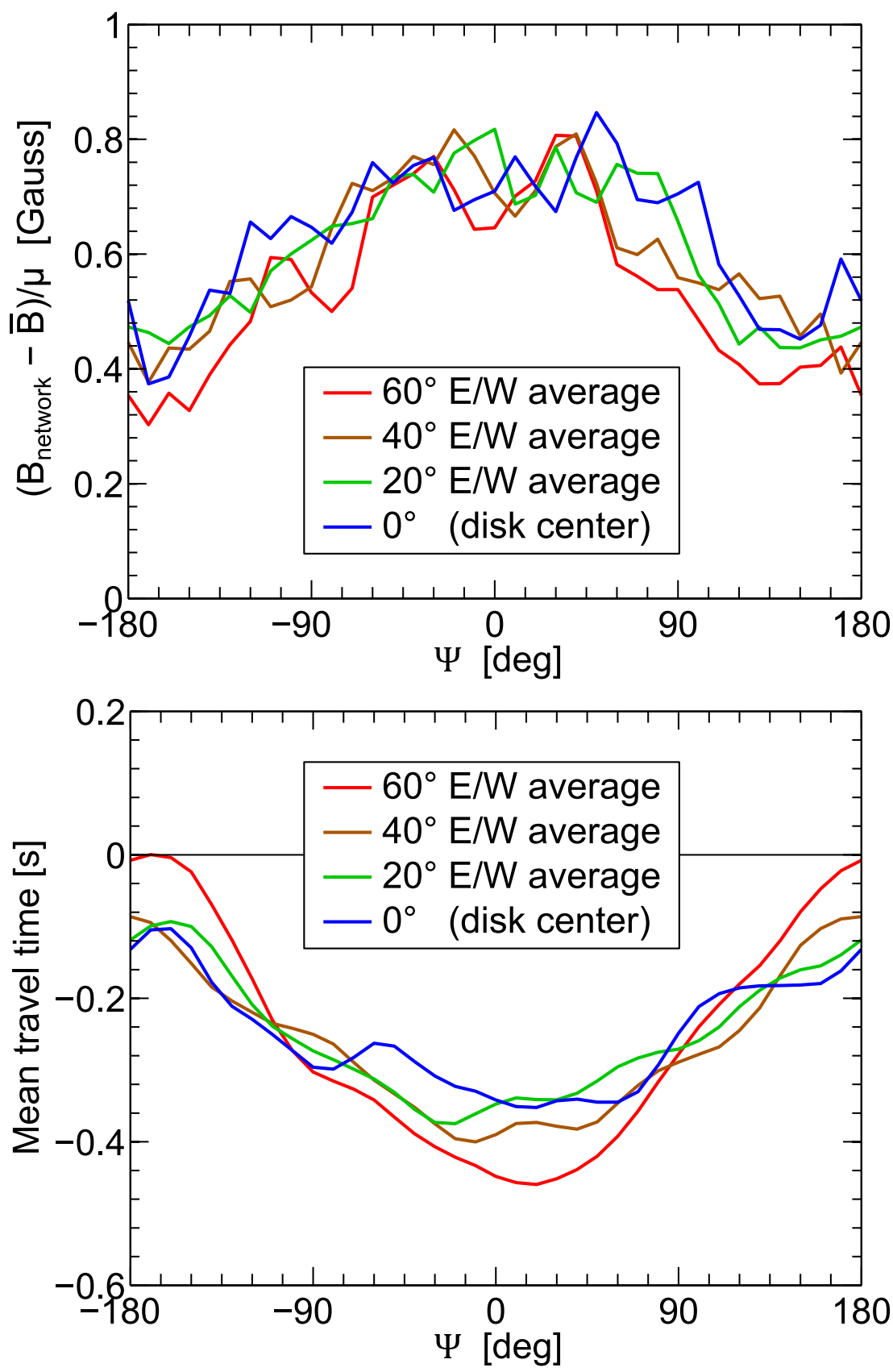

Figure C.6: Top: Network magnetic field in ring around the average supergranule depicted in Figs. C.4 and 4.2 (the inner and outer limits of the ring are 11 and $18 \mathrm{Mm}$ ) as a function of azimuthal angle $\psi$. The spatially averaged $\bar{B}$ was subtracted and the residual magnetic field was divided by $\mu$ (cosine of the heliocentric angle) to correct for projection effects (the assumption is that the network field is vertical). Bottom: As above, but for f-mode mean travel times (Figs. C.5 and 4.2). 


\section{C.4 Magnetic field at the average supergranular inflow}

As the counterpart of the magnetic field at the average supergranular outflow that was shown in Fig. 4.2, Fig. C.7 shows maps of the magnetic field and mean travel times at the average supergranular inflow near disk center. Figure C.8 shows the profile of the magnetic field and mean travel times along the $x$ axis, averaged over a strip $|y| \leq 2.5 \mathrm{Mm}$ (analogous to Fig. 4.3).
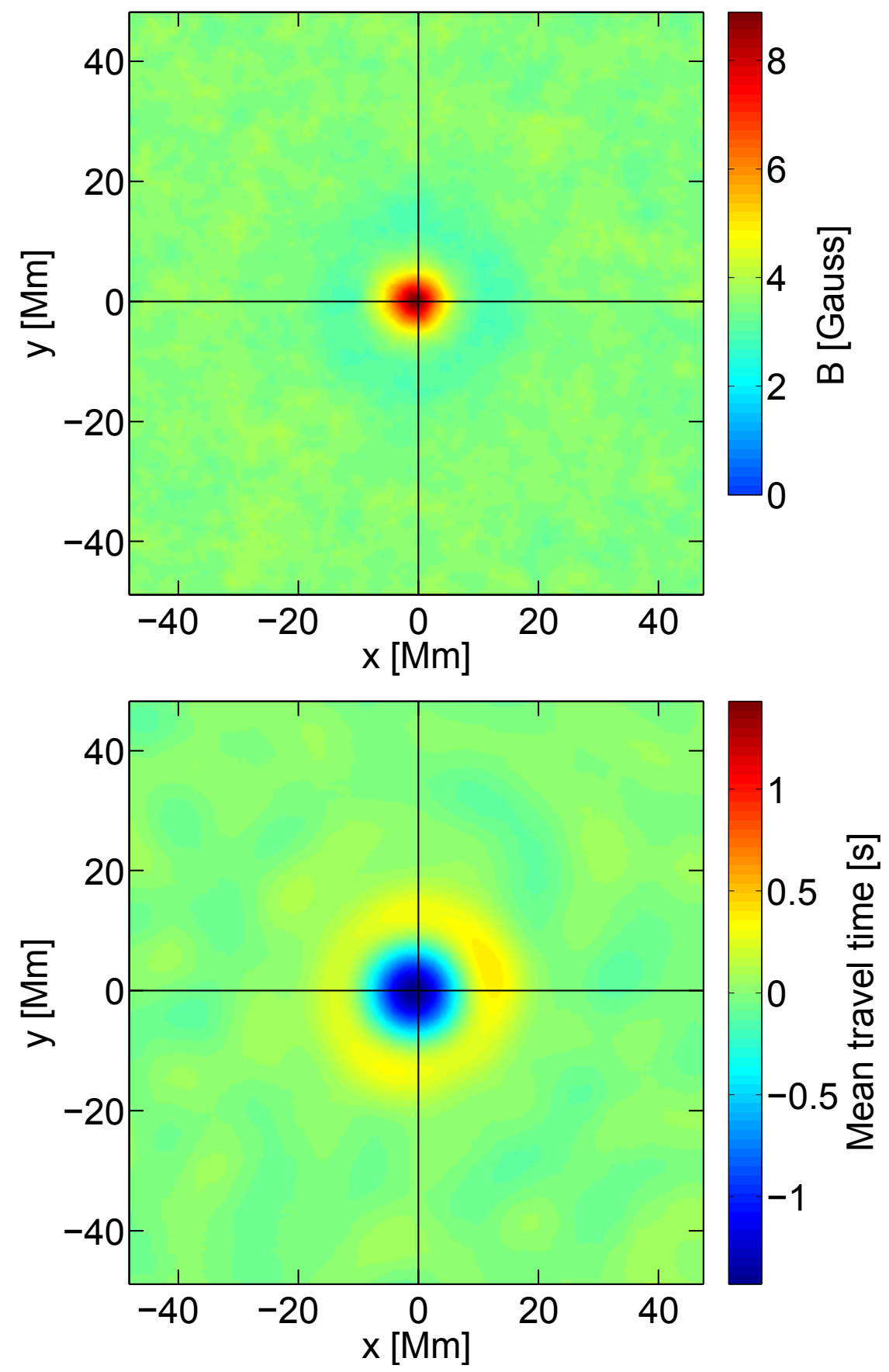

Figure C.7: As Fig. 4.2, but for the average supergranular inflow. Note that the color bar in the top panel was cut at zero because $B$ cannot be negative by construction. 

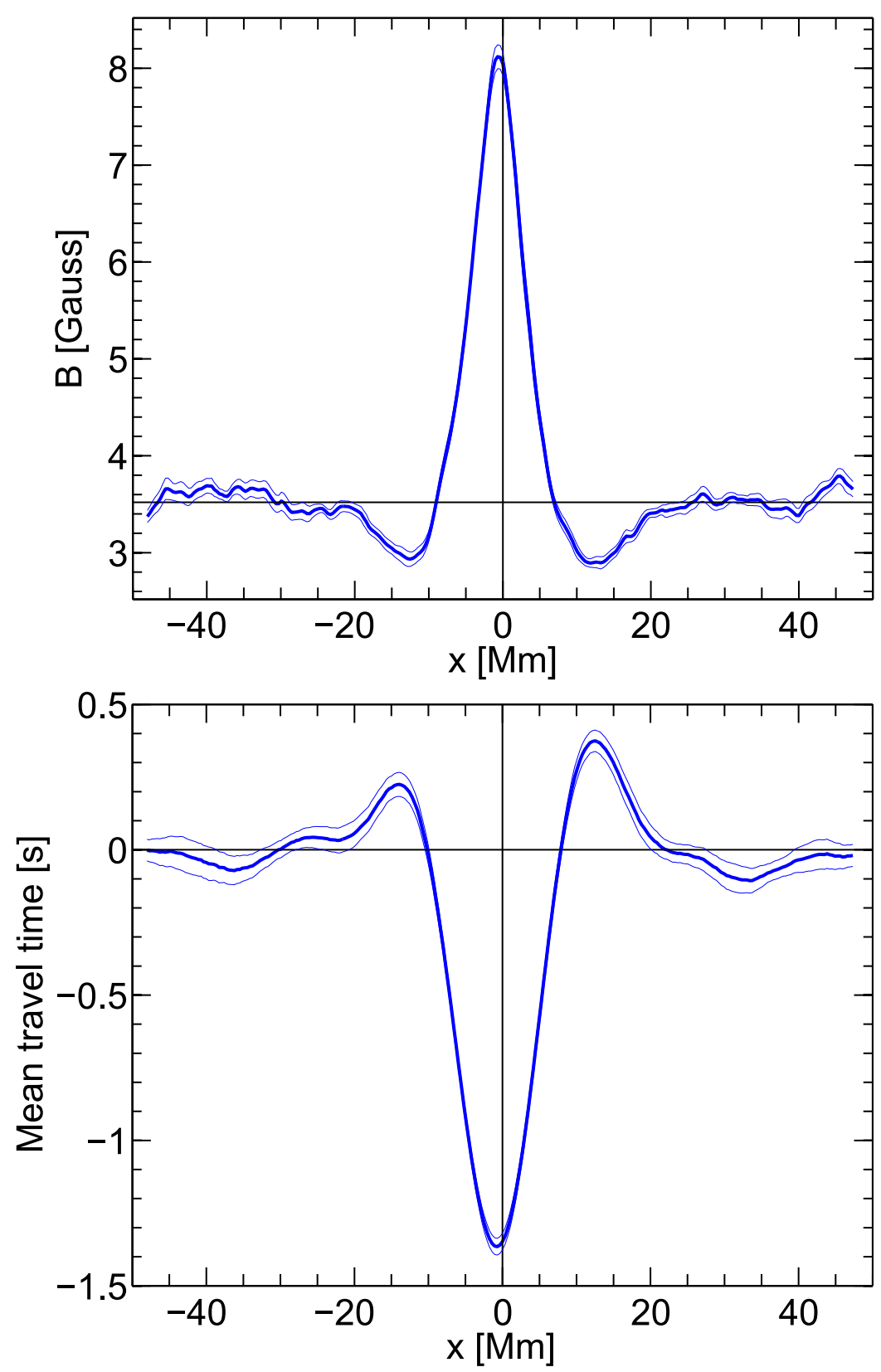

Figure C.8: As Fig. 4.3, but for the average supergranular inflow. 


\section{Publications}

\section{Refereed publications}

- J. Langfellner, L. Gizon, A. C. Birch: Time-distance helioseismology: A new averaging scheme for measuring flow vorticity, Astronomy \& Astrophysics 570, A90, 2014

- J. Langfellner, L. Gizon, A. C. Birch: Anisotropy of the solar network magnetic field around the average supergranule, Astronomy \& Astrophysics 579. L7, 2015

- J. Langfellner, L. Gizon, A. C. Birch: Spatially resolved vertical vorticity in solar supergranulation using helioseismology and local correlation tracking, Astronomy \& Astrophysics 581, A67, 2015

\section{Conference contributions}

- Stellar and Planetary Dynamos, Göttingen, Germany, 26-29 May 2015

Talk: Solar turbulent convection at supergranulation scale.

- HELAS VI/SOHO 28/SPACEINN meeting, Göttingen, Germany, 1-5 September 2014

Talk: Spatially resolved vorticity in supergranulation with helioseismology.

- LWS Solar Dynamics Observatory Science Workshop, Cambridge, Maryland, USA, 3-8 March 2013

Talk: Probing vortical motions in the Sun with time-distance helioseismology.

- Rocks 'n' Stars conference, Göttingen, Germany, 8-11 October 2012

Poster: Probing solar convection with helioseismology. 



\section{Acknowledgements/Danksagungen}

This work would not have been possible without the support from various people.

First, I'd like to thank my adviser Laurent Gizon for his continuous and tireless support and mentoring during the last years despite his manifold duties as a Max Planck director. I also thank Aaron for the collaboration on the papers, his many thoughts and ideas, and his comments on the manuscript. Furthermore, I'd like to thank Jesper, Tom, Robert, Hannah, Ray, and all the others who work in our group for the fruitful discussions and great working atmosphere. This includes my fellow $\mathrm{PhD}$ students who have shared the many ups and downs that inevitably occur over the course of a $\mathrm{PhD}$ project. I have learned a lot during the $\mathrm{PhD}$, and you guys made it enjoyable.

I'd also like to thank Stefan and Robert as members of my TAC, Damien and Thorsten for the collaboration in the CRC, Hannah and Ray for enduring my endless questions and requests regarding the mapping and tracking code, and Benjamin for providing his LaTeX template.

A special thanks goes to Sonja for her help and commitment in dealing with the bureaucratic framework and details.

Finally, I am indebted to the SDO/HMI team for having created such a marvelous instrument, making the work with its data a joyful experience.

Darüberhinaus möchte ich ganz besonders meinen Eltern danken, durch deren Unterstützung in allen Lebenslagen ich erst so weit gekommen bin.

Und auch meinen Freunden gebührt Dank für die nötige Ablenkung, die ab und an erforderlich ist, um solch eine Arbeit durchzuführen. 